\title{
Research Article \\ On Modelling of Two-Wire Transmission Lines with Uniform Passive Ladders
}

\author{
D. B. Kandić, ${ }^{1}$ B. D. Reljin, ${ }^{2}$ and I. S. Reljin ${ }^{3}$ \\ ${ }^{1}$ Department of Physics E Electrical Engineering, School of Mechanical Engineering, \\ University of Belgrade, Kraljice Marije 16, 11120 Belgrade, Serbia \\ ${ }^{2}$ Department of General Electrical Engineering, School of Electrical Engineering, University of Belgrade, \\ Bulevar Kralja Aleksandra 73, 11000 Belgrade, Serbia \\ ${ }^{3}$ Department of Telecommunications, School of Electrical Engineering, University of Belgrade, \\ Bulevar Kralja Aleksandra 73, 11000 Belgrade, Serbia \\ Correspondence should be addressed to D. B. Kandić, dbkandic@gmail.com
}

Received 23 March 2012; Accepted 1 May 2012

Academic Editor: Kue-Hong Chen

Copyright (C) 2012 D. B. Kandić et al. This is an open access article distributed under the Creative Commons Attribution License, which permits unrestricted use, distribution, and reproduction in any medium, provided the original work is properly cited.

\begin{abstract}
In the paper we presented new results in incremental network modelling of two-wire lines in frequency range $[0,3][\mathrm{GHz}]$, by the uniform $R L C G$ ladders with frequency dependent $R L$ parameters, which are analyzed by using PSPICE. Some important frequency limitations of the proposed approach have been pinpointed, restricting the application of developed models to steady-state analysis of RLCG networks transmitting the limited-frequency-band signals. The basic intention of this approach is to circumvent solving of telegraph equations or application of other complex, numerically demanding procedures in determining line steady-state responses at selected equidistant points. The key to the modelling method applied is partition of the two-wire line in segments with defined maximum length, whereby a couple of new polynomial approximations of line transcendental functions is introduced. It is proved that the strict equivalency between the short-line segments and their uniform ladder counterparts does not exist, but if some conditions are met, satisfactory approximations could be produced. This is illustrated by several examples of short and moderately long two-wire lines with different terminations, proving the good agreement between the exactly obtained steady-state results and those obtained by PSPICE simulation.
\end{abstract}

\section{Introduction}

A comprehensive theory of linear, time-invariant, lumped-parameter networks is presented in many references, where the physical dimensions of network elements are assumed small, compared to the wavelength associated with the highest frequency in the spectrum of the signal being processed. In those networks, two-terminal passive elements, such as resistors, capacitors, and inductors, are specified by single, spatially independent parameters. In passive electrical networks there may be also four-terminal elements, such as transformers and gyrators. The equilibrium equations of linear, time-invariant, lumped-parameter 
networks are ordinary, linear differential equations with constant coefficients. Unfortunately, all physical components cannot be treated as lumped, since their spatial configuration plays important role in understanding their physical behaviour at high frequencies. The systems such as electrical transmission lines, passive integrated circuits, as well as many physical processes: thermal conduction in rods, carrier motion in transistors, vibration of strings, and so forth, are characterized by partial differential equations, and distributed parameters must be introduced for correct mathematical description of their physical behaviour. Equilibrium equations of those distributed-parameter systems (i.e., partial differential equations) have solutions which are more difficult to find than the solutions of ordinary differential equations with constant coefficients. Since differential equations of transmission lines are analogous to those of many other systems and one-dimensional physical processes (e.g., of the heat flow in solids), in this paper we will: (a) make brief overview of partial differential equations describing voltage and current distributions in finite length lines, (b) develop the appropriate physical model of two-wire line with frequency dependent per-unit-length parameters by taking into account all the physically relevant parameters (geometry, dielectric, magnetic, and conductivity properties of media, the operating frequency range, and skin-effect), and (c) propose an approximate representation of real two-wire lines by uniform $R L C$ ladders with frequency-dependent parameters, turning thuswith the problem of analysis of real twowire lines into the analysis problem of high-order passive RLC networks by extensive use of PSPICE.

\section{The Incremental Network Model of Two-Wire Line}

In engineering practice the most widely and frequently used types of transmission lines are: (a) two-wire line (Figure 1(a)), coaxial line (Figure 1(b)) and twisted pair (Figure 1(c)). In Figure 1 with $\varepsilon_{c}, \mu_{c}$ and $\sigma_{c}$ or $\varepsilon_{d}, \mu_{d}$, and $\sigma_{d}$ are denoted: the electric permittivity, the magnetic permeability, and the specific electric conductivity of conductor (" $c$ ") or dielectric $(" d$ "), respectively. Nevertheless, no matter what type of transmission line is considered, each line segment (section) with physical length $\delta x$, which is sufficiently small compared to the wavelength associated with highest frequency in the spectrum of the signal being transmitted, can be represented with approximate, incremental, lumped network model depicted in Figure 2. Thereon are denoted with $R^{\prime}[\Omega / \mathrm{m}], L^{\prime}[\mathrm{H} / \mathrm{m}], C^{\prime}[\mathrm{F} / \mathrm{m}]$, and $G^{\prime}[S / m]$ : the resistance, inductance, capacitance, and conductance, respectively, of transmission line, in per-unit-length form. The lumped network model in Figure 2 becomes more and more accurate as $\delta x \rightarrow 0$, and it is proved to be an adequate representation of any transmission line, since it is in good agreement with the experimental observations. Throughout the paper the length of the line will be denoted by $\ell$ and the considered frequency range will be $f \in[0,3][\mathrm{GHz}]$. Dielectrics are assumed isotropic, linear, and homogeneous and, if imperfect, linear in ohmic sense, with constant specific electric conductivity $\sigma_{d} \ll \sigma_{c}$.

By neglecting the proximity effect with assumption $d \gg 2 a$, edge effect and taking $\sigma_{d} \ll \sigma_{c}$, the per-unit-length capacitance $C^{\prime}$ and the per-unit-length dielectric conductance $G^{\prime}$ of two-wire line in Figure 1(a) are calculated according to the following relations [1]:

$$
C^{\prime}=\frac{\pi \cdot \varepsilon_{d}}{\ln (d / a)}, \quad G^{\prime}=\frac{\sigma_{d}}{\varepsilon_{d}} \cdot C^{\prime}=\frac{\pi \cdot \sigma_{d}}{\ln (d / a)}, \quad\left(d \gg 2 a \wedge \sigma_{d} \ll \sigma_{c}\right) .
$$

It has been proved $[2,3]$ that due to the influence of the skin-effect each conductor of the two-wire line should be characterized by the frequency-dependent per-unit-length 


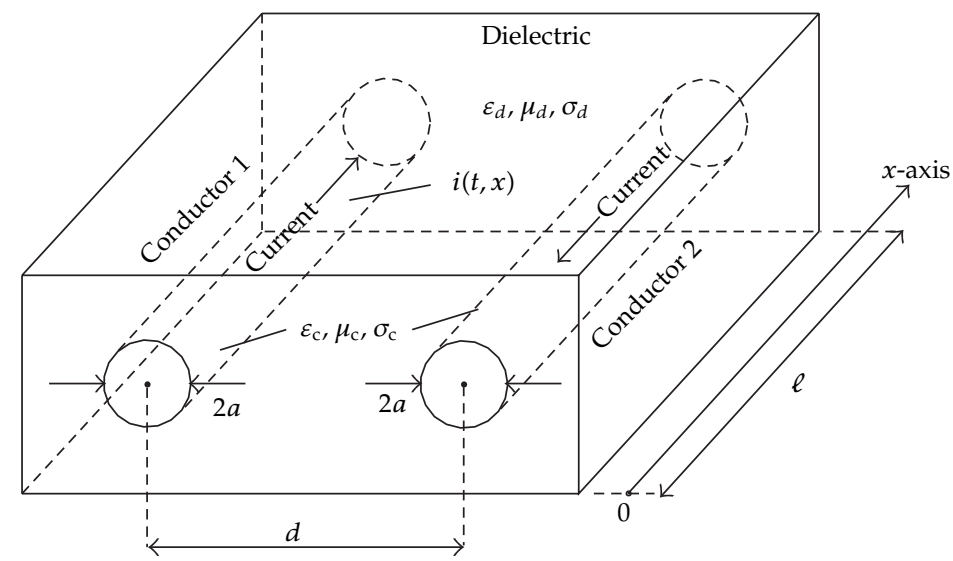

(a)

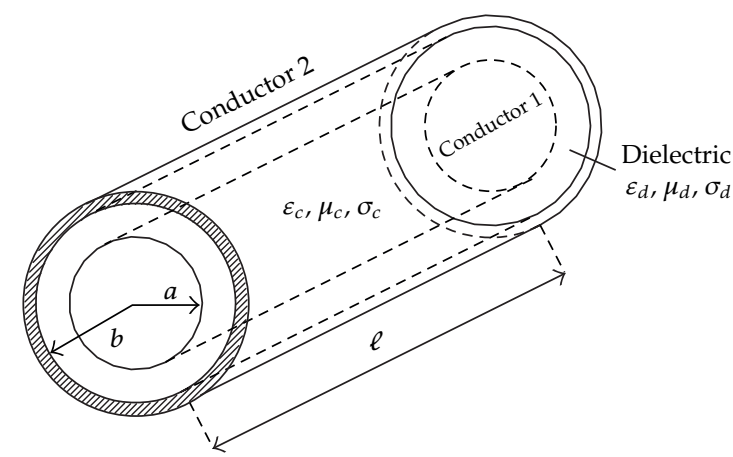

(b)

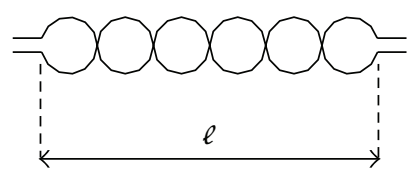

(c)

Figure 1: (a) Two-wire line, (b) coaxial line, and (c) twisted pair.

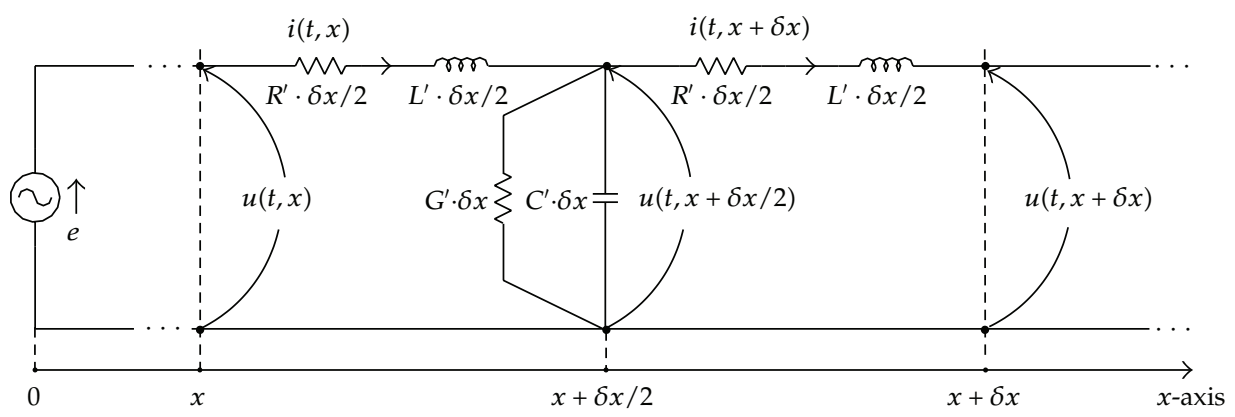

Figure 2: The approximate, incremental, and lumped network model of the uniform transmission line.

resistance $R_{i}^{\prime}(f)$ and the frequency-dependent per-unit-length inner inductance $L_{i}^{\prime}(f)$-for $f \in[0, \infty)[\mathrm{Hz}]$,

$$
\begin{aligned}
R_{i}^{\prime}(f) & =\frac{k}{2 \pi \cdot a \cdot \sigma_{c}} \cdot \operatorname{Re}\left[\frac{-\operatorname{bei}(k \cdot a)+j \cdot \operatorname{ber}(k \cdot a)}{\operatorname{ber}^{\prime}(k \cdot a)+j \cdot \operatorname{bei}^{\prime}(k \cdot a)}\right] \\
& =\frac{k}{2 \pi \cdot a \cdot \sigma_{c}} \cdot \frac{\operatorname{ber}(k \cdot a) \cdot \operatorname{bei}^{\prime}(k \cdot a)-\operatorname{bei}(k \cdot a) \cdot \operatorname{ber}^{\prime}(k \cdot a)}{\left[\operatorname{ber}^{\prime}(k \cdot a)\right]^{2}+\left[\operatorname{bei}^{\prime}(k \cdot a)\right]^{2}},
\end{aligned}
$$




$$
\begin{aligned}
L_{i}^{\prime}(f) & =\frac{k}{2 \pi \cdot a \cdot \sigma_{c} \cdot \omega} \cdot \operatorname{Im}\left[\frac{-\operatorname{bei}(k \cdot a)+j \cdot \operatorname{ber}(k \cdot a)}{\operatorname{ber}^{\prime}(k \cdot a)+j \cdot \operatorname{bei}^{\prime}(k \cdot a)}\right] \\
& =\frac{k}{2 \pi \cdot a \cdot \sigma_{c} \cdot \omega} \cdot \frac{\operatorname{ber}(k \cdot a) \cdot \operatorname{ber}^{\prime}(k \cdot a)+\operatorname{bei}(k \cdot a) \cdot \operatorname{bei}^{\prime}(k \cdot a)}{\left[\operatorname{ber}^{\prime}(k \cdot a)\right]^{2}+\left[\operatorname{bei}^{\prime}(k \cdot a)\right]^{2}},
\end{aligned}
$$

where: $j=\sqrt{-1}, \omega=2 \pi \cdot f, k=\sqrt{\omega \cdot \mu_{c} \cdot \sigma_{c}}$, and "Bessel real" $\operatorname{ber}(k \cdot a)$ and "Bessel imaginary" bei $(k \cdot a)$ are the Bessel-Kelvin functions with the first-order derivatives $\operatorname{ber}^{\prime}(k \cdot a)$ and $\operatorname{bei}^{\prime}(k \cdot a)$, respectively, at the point $z=k \cdot a$, which can be approximated at high frequencies (i.e., for $k \cdot a \gg 1$ ) with [4],

$$
\begin{gathered}
\operatorname{ber}(k \cdot a)=G \cdot \cos \left(\frac{k \cdot a}{\sqrt{2}}-\frac{\pi}{8}\right), \quad \operatorname{bei}(k \cdot a)=G \cdot \sin \left(\frac{k \cdot a}{\sqrt{2}}-\frac{\pi}{8}\right), \\
G=\frac{1}{\sqrt{2 \pi \cdot k \cdot a}} \cdot e^{(k \cdot a) / \sqrt{2},} \\
\operatorname{ber}^{\prime}(k \cdot a)=G \cdot \cos \left(\frac{k \cdot a}{\sqrt{2}}+\frac{\pi}{8}\right), \quad \operatorname{bei}^{\prime}(k \cdot a)=G \cdot \sin \left(\frac{k \cdot a}{\sqrt{2}}+\frac{\pi}{8}\right) .
\end{gathered}
$$

From (2.3) it should be firstly noticed that at high frequencies (i.e., for $k \cdot a \gg 1$ ) it holds

$$
\begin{aligned}
\frac{-\operatorname{bei}(k \cdot a)+j \cdot \operatorname{ber}(k \cdot a)}{\operatorname{ber}^{\prime}(k \cdot a)+j \cdot \operatorname{bei}^{\prime}(k \cdot a)} & =\frac{-\sin ((k \cdot a) / \sqrt{2}-\pi / 8)+j \cdot \cos ((k \cdot a) / \sqrt{2}-\pi / 8)}{\cos ((k \cdot a) / \sqrt{2}+\pi / 8)+j \cdot \sin ((k \cdot a) / \sqrt{2}+\pi / 8)} \\
& =j \cdot \frac{e^{j \cdot((k \cdot a) / \sqrt{2}-\pi / 8)}}{e^{j \cdot((k \cdot a) / \sqrt{2}+\pi / 8)}}=e^{j \cdot(\pi / 4)},
\end{aligned}
$$

and then from (2.2) it may be obtained consecutively for $k \cdot a \gg 1$,

$$
\begin{gathered}
R_{i}^{\prime}(f)=\frac{k}{2 \pi \cdot \sqrt{2} \cdot a \cdot \sigma_{c}}=\frac{1}{2 \pi \cdot a} \cdot \sqrt{\frac{\pi \cdot f \cdot \mu_{c}}{\sigma_{c}}}, \\
L_{i}^{\prime}(f)=\frac{k}{2 \pi \cdot \sqrt{2} \cdot \omega \cdot a \cdot \sigma_{c}}=\frac{1}{4 \pi \cdot a} \cdot \sqrt{\frac{\mu_{c}}{\pi \cdot f \cdot \sigma_{c}}} .
\end{gathered}
$$

The overall per-unit-length resistance $R^{\prime}(f)$ and the inductance $L^{\prime}(f)$ of two-wire line are

$$
\begin{gathered}
R^{\prime}(f)=\left.2 \cdot R_{i}^{\prime}(f)\right|_{\forall f^{\prime}} \quad R^{\prime}(f)=\frac{1}{\pi \cdot a} \cdot \sqrt{\frac{\pi \cdot f \cdot \mu_{c}}{\sigma_{c}}}=\left.\frac{R_{s}(f)}{\pi \cdot a}\right|_{k \cdot a \gg 1}, \quad R_{s}(f)=\sqrt{\frac{\pi \cdot f \cdot \mu_{c}}{\sigma_{c}}}, \\
L^{\prime}(f)=L_{e}^{\prime}+\left.2 \cdot L_{i}^{\prime}(f)\right|_{\forall f^{\prime}} \quad L^{\prime}(f)=\frac{\mu_{d}}{\pi} \cdot \ln \left(\frac{d}{a}\right)+\left.\frac{1}{2 \pi \cdot a} \cdot \sqrt{\frac{\mu_{c}}{\pi \cdot f \cdot \sigma_{c}}}\right|_{k \cdot a \gg 1} \quad(d \gg 2 a),
\end{gathered}
$$


where $R_{s}(f)$ is the surface resistance of line conductors and $L_{e}^{\prime}=\left(\mu_{d} / \pi\right) \cdot \ln (d / a)$ is the external per-unit-length inductance of two-wire line. Throught the paper it will be assumed that $\mu_{c} \approx \mu_{d} \approx \mu_{0}$. $k \cdot a)[4]$

Since the following expansions hold for any frequency $f \in[0, \infty)[\mathrm{Hz}]$ (i.e., for any

$$
\begin{aligned}
& \operatorname{ber}(k \cdot a)=\sum_{n=0}^{\infty}(-1)^{n} \cdot \frac{(k \cdot a / 2)^{4 n}}{(2 n !)^{2}}=1-\frac{(k \cdot a)^{4}}{2^{2} \cdot 4^{2}}+\frac{(k \cdot a)^{8}}{2^{2} \cdot 4^{2} \cdot 6^{2} \cdot 8^{2}} \\
& -\frac{(k \cdot a)^{12}}{2^{2} \cdot 4^{2} \cdot 6^{2} \cdot 8^{2} \cdot 10^{2} \cdot 12^{2}} \pm \ldots, \\
& \operatorname{bei}(k \cdot a)=\sum_{n=1}^{\infty}(-1)^{n+1} \cdot \frac{(k \cdot a / 2)^{4 n-2}}{[(2 n-1) !]^{2}}=\frac{(k \cdot a)^{2}}{2^{2}}-\frac{(k \cdot a)^{6}}{2^{2} \cdot 4^{2} \cdot 6^{2}} \\
& +\frac{(k \cdot a)^{10}}{2^{2} \cdot 4^{2} \cdot 6^{2} \cdot 8^{2} \cdot 10^{2}} \mp \ldots, \\
& \operatorname{ber}^{\prime}(k \cdot a)=-\frac{(k \cdot a)^{3}}{2^{2} \cdot 4}+\frac{(k \cdot a)^{7}}{2^{2} \cdot 4^{2} \cdot 6^{2} \cdot 8}-\frac{(k \cdot a)^{11}}{2^{2} \cdot 4^{2} \cdot 6^{2} \cdot 8^{2} \cdot 10^{2} \cdot 12} \\
& +\frac{(k \cdot a)^{15}}{2^{2} \cdot 4^{2} \cdot 6^{2} \cdot 8^{2} \cdot 10^{2} \cdot 12^{2} \cdot 14^{2} \cdot 16} \mp \ldots, \\
& \operatorname{bei}^{\prime}(k \cdot a)=\frac{k \cdot a}{2}-\frac{(k \cdot a)^{5}}{2^{2} \cdot 4^{2} \cdot 6}+\frac{(k \cdot a)^{9}}{2^{2} \cdot 4^{2} \cdot 6^{2} \cdot 8^{2} \cdot 10} \\
& -\frac{(k \cdot a)^{13}}{2^{2} \cdot 4^{2} \cdot 6^{2} \cdot 8^{2} \cdot 10^{2} \cdot 12^{2} \cdot 14} \pm \ldots,
\end{aligned}
$$

then by using (2.2) and (2.7) when $f \rightarrow 0[\mathrm{~Hz}]$, the following consequences are easily obtained: $R_{i}^{\prime}(f) \rightarrow 1 /\left(\sigma_{c} \cdot \pi \cdot a^{2}\right), L_{i}^{\prime}(f) \rightarrow \mu_{c} /(8 \cdot \pi), R^{\prime}(f) \rightarrow 2 /\left(\sigma_{c} \cdot \pi \cdot a^{2}\right), L^{\prime}(f) \rightarrow$ $\left(\mu_{d} / \pi\right) \cdot \ln (d / a)+\mu_{c} /(4 \cdot \pi)$. Automatic, fast, and accurate numerical calculation of BesselKelvin functions (2.7) imposes a real need to distinguish between the following two cases of approximation, depending on magnitude of $z=k \cdot a \in[0, \infty)$ [5],

Case $A\left(z=z(f)=a \cdot k=a \cdot \sqrt{2 \cdot \pi \cdot f \cdot \mu_{c} \cdot \sigma_{c}} \in[0,8]\right)$.

$$
\begin{aligned}
\operatorname{ber}(z)= & -64 \cdot\left(\frac{z}{8}\right)^{4}+113.77777774 \cdot\left(\frac{z}{8}\right)^{8}-32.36345652 \cdot\left(\frac{z}{8}\right)^{12} \\
& +2.64191397 \cdot\left(\frac{z}{8}\right)^{16}-0.08349609 \cdot\left(\frac{z}{8}\right)^{20}+0.00122552 \cdot\left(\frac{z}{8}\right)^{24} \\
& -0.00000901 \cdot\left(\frac{z}{8}\right)^{28}+\varepsilon_{1} \quad\left(\left|\varepsilon_{1}\right|<10^{-9}\right), \\
\operatorname{bei}(z)= & 16 \cdot\left(\frac{z}{8}\right)^{2}-113.77777774 \cdot\left(\frac{z}{8}\right)^{6}+72.81777742 \cdot\left(\frac{z}{8}\right)^{10}-10.56765779 \cdot\left(\frac{z}{8}\right)^{14} \\
& +0.52185615 \cdot\left(\frac{z}{8}\right)^{18}-0.01103667 \cdot\left(\frac{z}{8}\right)^{22} \\
& +0.00011346 \cdot\left(\frac{z}{8}\right)^{26}+\varepsilon_{2} \quad\left(\left|\varepsilon_{2}\right|<6 \cdot 10^{-9}\right),
\end{aligned}
$$




$$
\begin{aligned}
\operatorname{ber}^{\prime}(z)= & z \cdot\left[-4 \cdot\left(\frac{z}{8}\right)^{2}+14.22222222 \cdot\left(\frac{z}{8}\right)^{6}-6.06814810 \cdot\left(\frac{z}{8}\right)^{10}+0.66047849 \cdot\left(\frac{z}{8}\right)^{14}\right. \\
& \left.-0.02609253 \cdot\left(\frac{z}{8}\right)^{18}+0.00045957 \cdot\left(\frac{z}{8}\right)^{22}-0.00000394 \cdot\left(\frac{z}{8}\right)^{26}\right] \\
+ & \varepsilon_{3} \quad\left(\left|\varepsilon_{3}\right|<2.1 \cdot 10^{-8}\right) \\
\operatorname{bei}^{\prime}(z)= & z \cdot\left[\frac{1}{2}-10.66666666 \cdot\left(\frac{z}{8}\right)^{4}+11.37777772 \cdot\left(\frac{z}{8}\right)^{8}-2.31167514 \cdot\left(\frac{z}{8}\right)^{12}\right. \\
& \left.+0.14677204 \cdot\left(\frac{z}{8}\right)^{16}-0.00379386 \cdot\left(\frac{z}{8}\right)^{20}+0.00004609 \cdot\left(\frac{z}{8}\right)^{24}\right] \\
+ & \varepsilon_{4} \quad\left(\left|\varepsilon_{4}\right|<7 \cdot 10^{-8}\right) .
\end{aligned}
$$

Case B $\left(z=z(f)=a \cdot k=a \cdot \sqrt{2 \cdot \pi \cdot f \cdot \mu_{c} \cdot \sigma_{c}} \in(8, \infty)\right)$.

Define firstly the following set of auxiliary functions:

$$
\begin{aligned}
\alpha(z)= & -0.3926991 \cdot j+(0.0110486-0.0110485 \cdot j) \cdot\left(\frac{8}{z}\right)-0.0009765 \cdot j \cdot\left(\frac{8}{z}\right)^{2} \\
& +(-0.0000906-0.0000901 \cdot j) \cdot\left(\frac{8}{z}\right)^{3}-0.0000252 \cdot\left(\frac{8}{z}\right)^{4} \\
& +(-0.0000034+0.0000051 \cdot j) \cdot\left(\frac{8}{z}\right)^{5}+(0.0000006+0.0000019 \cdot j) \cdot\left(\frac{8}{z}\right)^{6} \\
\alpha(-z)= & -0.3926991 \cdot j+(-0.0110486+0.0110485 \cdot j) \cdot\left(\frac{8}{z}\right)-0.0009765 \cdot j \cdot\left(\frac{8}{z}\right)^{2} \\
& +(0.0000906+0.0000901 \cdot j) \cdot\left(\frac{8}{z}\right)^{3}-0.0000252 \cdot\left(\frac{8}{z}\right)^{4} \\
& +(0.0000034-0.0000051 \cdot j) \cdot\left(\frac{8}{z}\right)^{5}+(0.0000006+0.0000019 \cdot j) \cdot\left(\frac{8}{z}\right)^{6} \\
\beta(z)= & (0.7071068+0.7071068 \cdot j)+(-0.0625001-0.0000001 \cdot j) \cdot\left(\frac{8}{z}\right) \\
& +(-0.0013813+0.0013811 \cdot j) \cdot\left(\frac{8}{z}\right)^{2}+(0.0000005+0.0002452 \cdot j) \cdot\left(\frac{8}{z}\right)^{3} \\
& +(0.0000346+0.0000338 \cdot j) \cdot\left(\frac{8}{z}\right)^{4}+(0.0000017-0.0000024 \cdot j) \cdot\left(\frac{8}{z}\right)^{5} \\
& +(0.0000016-0.0000032 \cdot j) \cdot\left(\frac{8}{z}\right)^{6}
\end{aligned}
$$


Mathematical Problems in Engineering

$$
\begin{aligned}
\beta(-z)= & (0.7071068+0.7071068 \cdot j)+(0.0625001+0.0000001 \cdot j) \cdot\left(\frac{8}{z}\right) \\
& +(-0.0013813+0.0013811 \cdot j) \cdot\left(\frac{8}{z}\right)^{2}+(-0.0000005-0.0002452 \cdot j) \cdot\left(\frac{8}{z}\right)^{3} \\
& +(0.0000346+0.0000338 \cdot j) \cdot\left(\frac{8}{z}\right)^{4}+(-0.0000017+0.0000024 \cdot j) \cdot\left(\frac{8}{z}\right)^{5} \\
& +(0.0000016-0.0000032 \cdot j) \cdot\left(\frac{8}{z}\right)^{6} .
\end{aligned}
$$

and, also, define another set of auxiliary functions:

$$
f(z)=\sqrt{\frac{\pi}{2 \cdot z}} \cdot \exp \left[-\frac{1+j}{\sqrt{2}} \cdot z+\alpha(-z)\right], \quad g(z)=\frac{1}{\sqrt{2 \cdot \pi \cdot z}} \cdot \exp \left[\frac{1+j}{\sqrt{2}} \cdot z+\alpha(z)\right]
$$

then, the values of Bessel-Kelvin functions can be efficiently calculated [5] by using the relations:

$$
\begin{array}{cc}
\operatorname{ber}(z)=\operatorname{Re}\left[\frac{j}{\pi} \cdot f(z)+g(z)\right], & \operatorname{bei}(z)=\operatorname{Im}\left[\frac{j}{\pi} \cdot f(z)+g(z)\right], \\
\operatorname{ber}^{\prime}(z)=\operatorname{Re}\left[-\frac{j}{\pi} \cdot f(z) \cdot \beta(-z)+g(z) \cdot \beta(z)\right], & \operatorname{bei}^{\prime}(z)=\operatorname{Im}\left[-\frac{j}{\pi} \cdot f(z) \cdot \beta(-z)+g(z) \cdot \beta(z)\right] .
\end{array}
$$

For the coaxial line with length $\ell$ (Figure $1(\mathrm{~b})$ ), the per-unit-length capacitance $C^{\prime}$ and the per-unit-length conductance $G^{\prime}$ of dielectric are calculated according to the following relations [1]:

$$
C^{\prime}=\frac{2 \pi \cdot \varepsilon_{d}}{\ln (b / a)}, \quad G^{\prime}=\frac{\sigma_{d}}{\varepsilon_{d}} \cdot C^{\prime}=\frac{2 \pi \cdot \sigma_{d}}{\ln (b / a)}, \quad\left(\sigma_{d} \ll \sigma_{c}\right) .
$$

It has been shown [1-3] that at high frequencies the coaxial line is characterized by the per-unit-length resistance $R^{\prime}(f)$ and the per-unit-length inductance $L^{\prime}(f)$ given with,

$$
\begin{gathered}
R^{\prime}(f)=\frac{R_{s}(f)}{2 \pi} \cdot\left(\frac{1}{a}+\frac{1}{b}\right)=\frac{1}{2 \pi} \cdot \sqrt{\frac{\pi \cdot f \cdot \mu_{c}}{\sigma_{c}}} \cdot\left(\frac{1}{a}+\frac{1}{b}\right), \\
L^{\prime} \approx L_{e}^{\prime}=\frac{\mu_{d}}{2 \pi} \cdot \ln \left(\frac{b}{a}\right), \quad\left(\mu_{c} \approx \mu_{d} \approx \mu_{0}\right) .
\end{gathered}
$$

The twisted-pair (Figure 1(c)) has characteristics similar to those of the two-wire line, except for the smaller inductivity and the smaller modulus $Z_{0}$ of its characteristic impedance $\underline{Z}_{0}[3,6]$.

To resume our investigation, consider two-wire line with copper conductors and polyethylene dielectric, where $a=0.1[\mathrm{~mm}]$ and $d=4[\mathrm{~mm}]$ (Figure 1(a)). Let the operating 


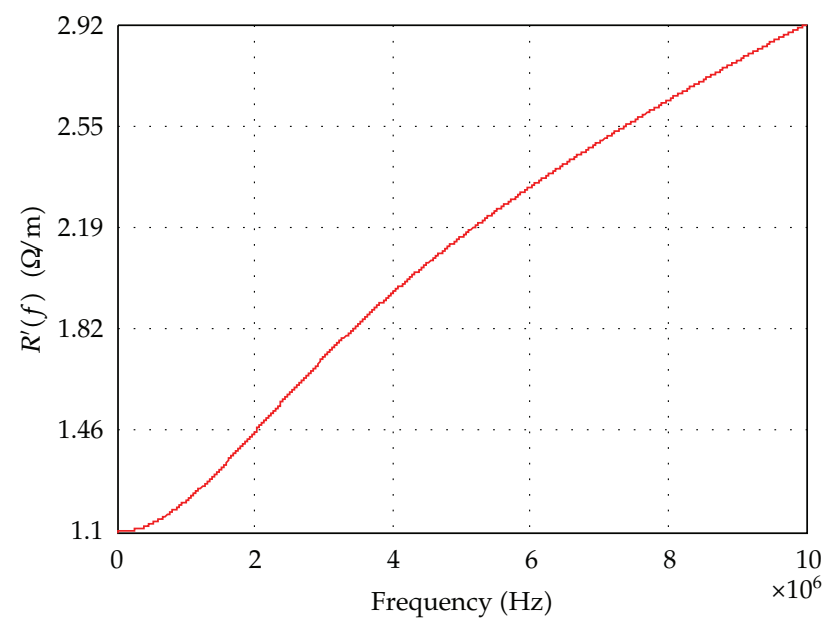

Figure 3: $R^{\prime}(f)$ for line with $a=0.1[\mathrm{~mm}]$ and $d=4[\mathrm{~mm}]$.

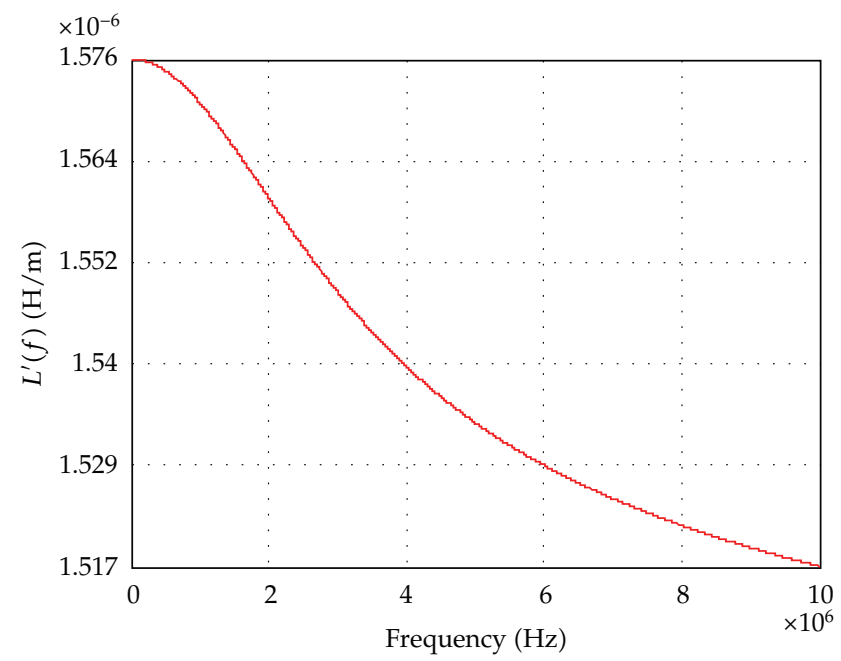

Figure 4: $L^{\prime}(f)$ for line with $a=0.1[\mathrm{~mm}]$ and $d=4[\mathrm{~mm}]$.

frequency range of this line be $f \in[0,3][\mathrm{GHz}]$. The specific electric conductivity of copper is $\sigma_{c} \approx 5.81 \cdot 10^{7}[\mathrm{~S} / \mathrm{m}]$ and magnetic permeability is $\mu_{c} \approx \mu_{0}$. At the temperature $T=298$ $[\mathrm{K}]$ the relative permittivity of polyethylene is $\varepsilon_{r} \approx 2.26$ (for frequencies up to $25[\mathrm{GHz}]$ ) and its specific electric conductivity is $\sigma_{d} \approx 10^{-15}[\mathrm{~S} / \mathrm{m}]$. From $(2.1)$ it is calculated $C^{\prime}=17.04$ $[\mathrm{pF} / \mathrm{m}]$ and $G^{\prime}=0.85[\mathrm{fS} / \mathrm{m}]$. At frequency $f=0[\mathrm{~Hz}]$ the per-unit-length resistance of this line is $R^{\prime}(0)=2 /\left(\sigma_{c} \cdot \pi \cdot a^{2}\right)=1.0957[\Omega / \mathrm{m}]$ and its per-unit-length inductance is $L^{\prime}(0)=\left(\mu_{d} / \pi\right) \cdot \ln (d / a)+\mu_{c} /(4 \cdot \pi) \approx 1.575[\mu \mathrm{H} / \mathrm{m}]$. At frequency $f=1[\mathrm{GHz}]$ it is calculated from $(2.2)$ and $(2.9)-(2.11): R^{\prime}(f)=26.514[\Omega / \mathrm{m}] \gg R^{\prime}(0)$ and $L^{\prime}(f) \approx 1.479[\mu \mathrm{H} / \mathrm{m}]$, and for the current wavelength it is obtained $\lambda \approx c_{0} /\left[f \cdot\left(\varepsilon_{r}\right)^{1 / 2}\right] \approx 20[\mathrm{~cm}]$. In Figures 3, 4, 5, and 6 the variations of $R^{\prime}(f), L^{\prime}(f), d R^{\prime}(f) / d f$, and $d L^{\prime}(f) / d f$ are depicted, respectively, in the frequency range $f \in[0,10][\mathrm{MHz}]$, whereas the variations of these quantities in the frequency range $f \in[0.01,3][\mathrm{GHz}]$ are depicted in Figures $7,8,9$, and 10, respectively. Let the frequency spectrum of the signal being transmitted is $\left[f_{0}-B / 2, f_{0}+B / 2\right]\left(f_{0}\right.$ is the central 


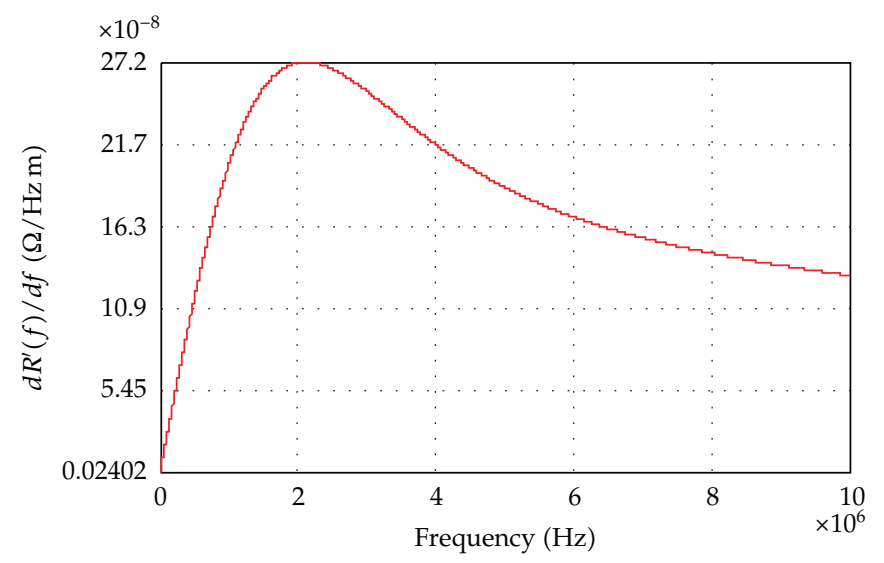

Figure 5: $d R^{\prime}(f) / d f$ for line with $a=0.1[\mathrm{~mm}]$ and $d=4[\mathrm{~mm}]$.

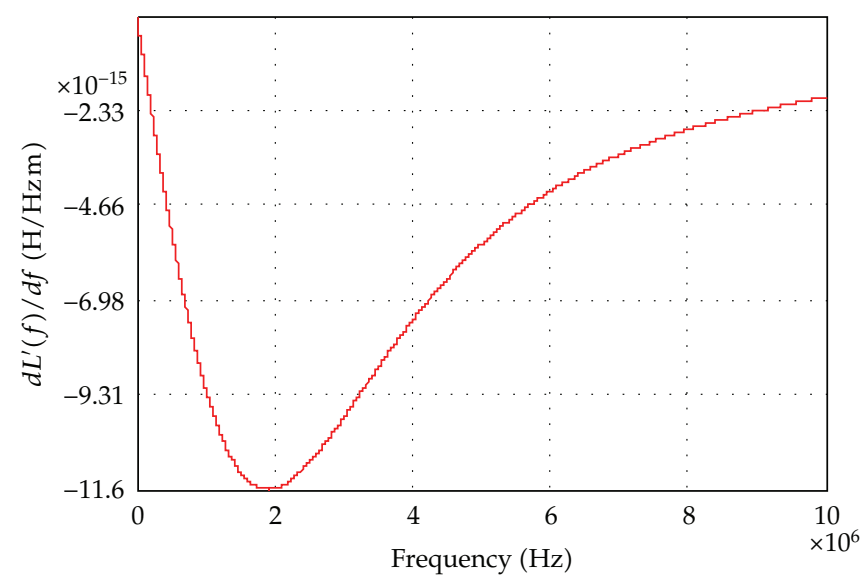

Figure 6: $d L^{\prime}(f) / d f$ for line with $a=0.1[\mathrm{~mm}]$ and $d=4[\mathrm{~mm}]$.

frequency of the signal spectrum and $B$ the signal bandwith). If the integral of function in Figure 5 taken between $f_{0}-B / 2$ and $f_{0}+B / 2$ is less than 0.01 , then we see from Figure 3 that it may be taken $R^{\prime}(f) \approx R^{\prime}\left(f_{0}\right)$, for $f \in\left[f_{0}-B / 2, f_{0}+B / 2\right]$. And by using Figure 5 we obtain in the most conservative approach that $B \leq 37$ [kHz]. Similarly, if the integral of function in Figure 9 taken between $f_{0}-B / 2$ and $f_{0}+B / 2$ is less than 0.1 , then we see from Figure 5 that it, also, holds $R^{\prime}(f) \approx R^{\prime}\left(f_{0}\right)$, for $f \in\left[f_{0}-B / 2, f_{0}+B / 2\right]$. And by using Figure 9 we obtain in the most conservative approach that $B \leq 770[\mathrm{kHz}]$.

For a lossless transmission line $\left(\Leftrightarrow R^{\prime}=0[\Omega / \mathrm{m}]\right.$ and $\left.G^{\prime}=0[\mathrm{~S} / \mathrm{m}]\right)$ with linear and homogeneous dielectric the phase-velocity $c$ of electromagnetic perturbation (i.e., the propagation speed of current wave in the line) and characteristic impedance $\underline{Z}_{0}$ are given by the following relations [1-3]:

$$
\begin{gathered}
c=\frac{1}{\sqrt{L^{\prime} \cdot C^{\prime}}}=\frac{1}{\sqrt{\varepsilon_{d} \cdot \mu_{d}}}=\frac{c_{0}}{\sqrt{\varepsilon_{r} \cdot \mu_{r}}}, \quad c_{0}=\frac{1}{\sqrt{\varepsilon_{0} \cdot \mu_{0}}} \approx 3 \cdot 10^{8}\left[\frac{\mathrm{m}}{\mathrm{s}}\right], \\
\underline{Z}_{0}=\sqrt{\frac{L^{\prime}}{C^{\prime}}}=\frac{1}{c \cdot C^{\prime}}=\sqrt{\frac{\mu_{r}}{\varepsilon_{r}}} \cdot \frac{1}{\pi} \cdot \sqrt{\frac{\mu_{0}}{\varepsilon_{0}}} \cdot \ln \left(\frac{d}{a}\right) \approx 120 \cdot \sqrt{\frac{\mu_{r}}{\varepsilon_{r}}} \cdot \ln \left(\frac{d}{a}\right)[\Omega],
\end{gathered}
$$




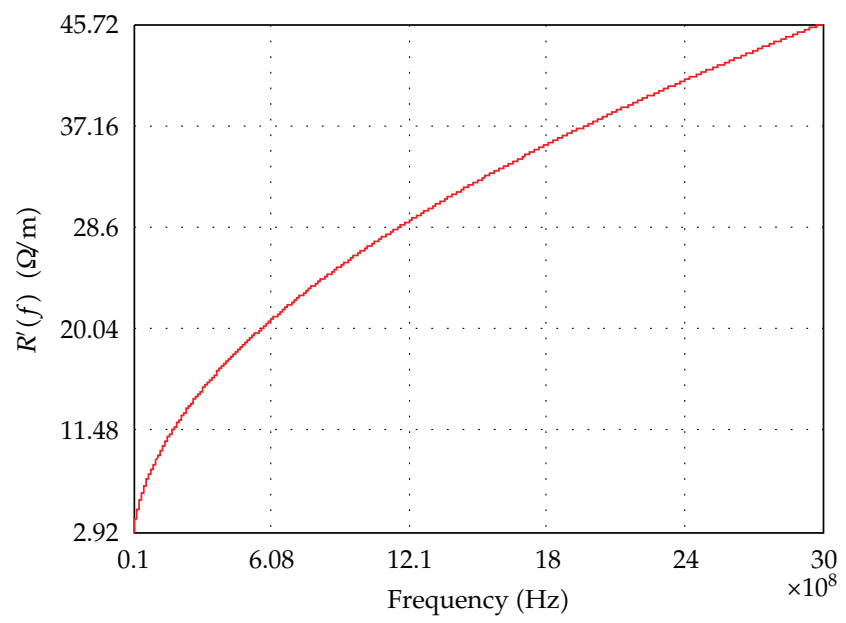

Figure 7: $R^{\prime}(f)$ for line with $a=0.1[\mathrm{~mm}]$ and $d=4[\mathrm{~mm}]$.

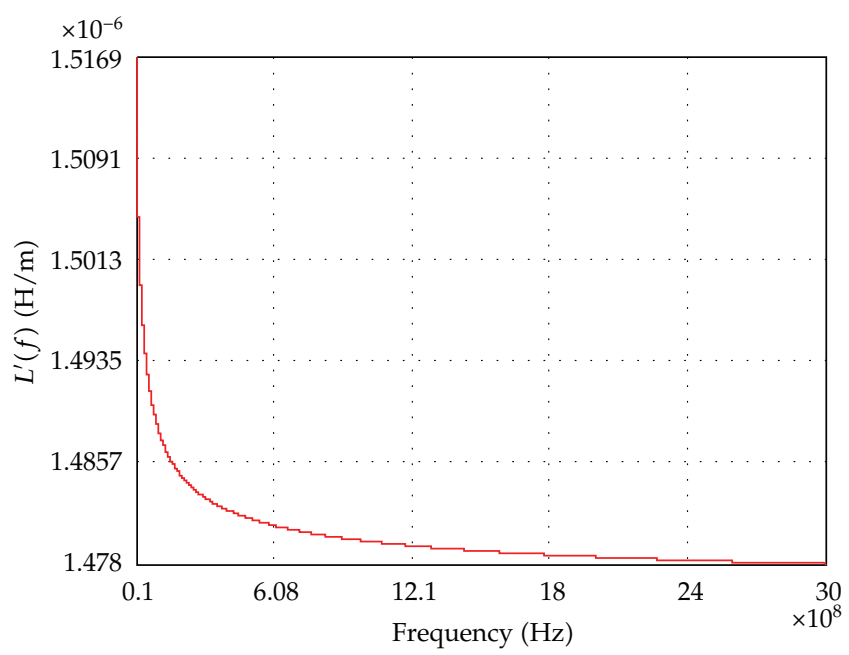

Figure 8: $L^{\prime}(f)$ for line with $a=0.1[\mathrm{~mm}]$ and $d=4[\mathrm{~mm}]$.

where $\varepsilon_{r}=\varepsilon_{d} / \varepsilon_{0}$ is relative permittivity and $\mu_{r}=\mu_{d} / \mu_{0}$ relative permeability of dielectric $\left(\varepsilon_{0} \approx 10^{-9} / 36 \pi[\mathrm{F} / \mathrm{m}]\right.$ is permittivity and $\mu_{0}=4 \pi \cdot 10^{-7}[\mathrm{H} / \mathrm{m}]$ permeability of vacuum). The characteristic impedance of a lossy transmission line is generally defined as $\underline{Z}_{0}(j \cdot 2 \pi \cdot f)=$ $\left[\left(R^{\prime}+j \cdot 2 \pi \cdot f \cdot L^{\prime}\right) /\left(G^{\prime}+j \cdot 2 \pi \cdot f \cdot C^{\prime}\right)\right]^{1 / 2}$. In the case considered, on Figures 11 and 12 the variations of $Z_{0}(f)=\left|\underline{Z}_{0}(j \cdot 2 \pi \cdot f)\right|$ and $\zeta(f)=\operatorname{Arg}\left[\underline{Z}_{0}(j \cdot 2 \pi \cdot f)\right]$ in the frequency range $f \in[0.01,3]$ $[\mathrm{GHz}]$ are respectively depicted. In older telephony applications at lower frequencies, $Z_{0}$ was typically $600[\Omega]$ for air two-wire lines. For symmetric antenna feeding at frequencies up to $500[\mathrm{MHz}]$, sometimes the two-wire lines with standard characteristic impedances $Z_{0}=240$ or $300[\Omega]$ are used. At shorter distances in telephony and local computer networks, nowdays are used the twisted-pairs (two-wire lines with reduced inductance) with standard $Z_{0}=100$ $[\Omega]$ and the propagation speed approximately $c_{0} / 2$. For the coaxial lines the standard $Z_{0}$ is 50 or $75[\Omega]$ and their propagation speed is approximately $2 c_{0} / 3$. For the printed transmission lines, $Z_{0}$ is in the range $100 \div 150[\Omega]$, and their propagation speed is approximately $c_{0} / 2[6]$. 


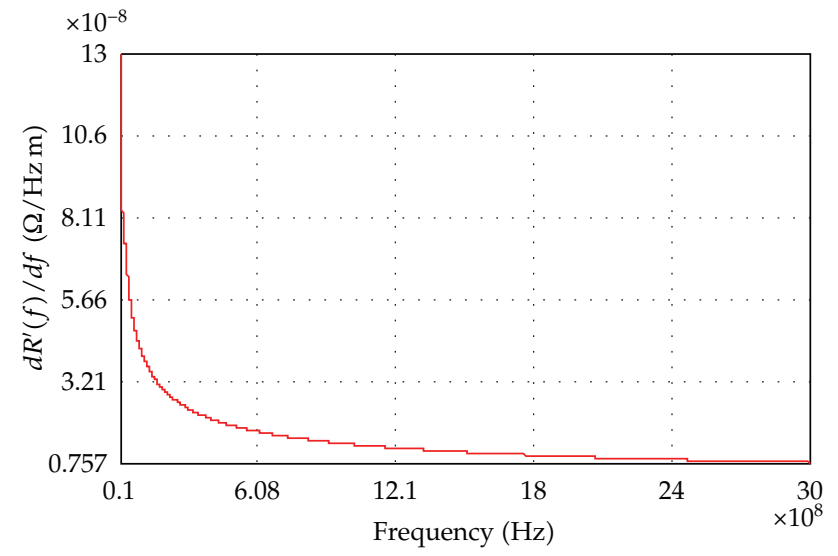

Figure 9: $d R^{\prime}(f) / d f$ for line with $a=0.1[\mathrm{~mm}]$ and $d=4[\mathrm{~mm}]$.

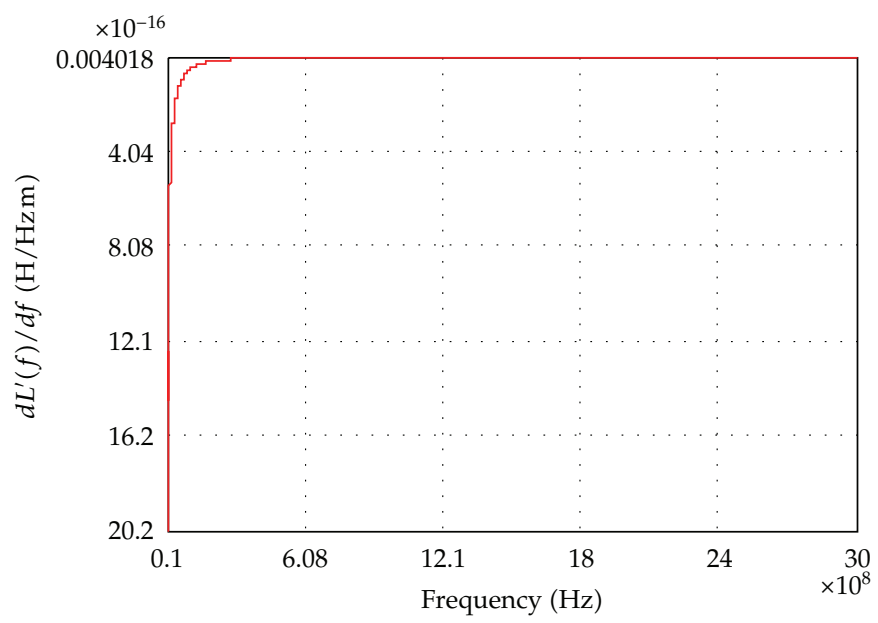

Figure 10: $d L^{\prime}(f) / d f$ for line with $a=0.1[\mathrm{~mm}]$ and $d=4[\mathrm{~mm}]$.

For two-wire line being considered, in Figures 13, 14, 15, and 16 variations of several functions in the frequency range $f \in[0,3][\mathrm{GHz}]$ are depicted, which will be used in later consideration,

$$
\begin{gathered}
\phi_{1}(f)=\frac{2 \cdot \pi \cdot f \cdot L^{\prime}(f)}{R^{\prime}(f)}, \quad \phi_{2}(f)=\frac{2 \cdot \pi \cdot f \cdot C^{\prime}}{G^{\prime}}, \quad \phi_{3}(f)=\frac{1}{2 \cdot \pi \cdot f \cdot \sqrt{L^{\prime}(f) \cdot C^{\prime}}}, \\
\phi_{4}(f)=\frac{C^{\prime} \cdot R^{\prime}(f)}{G^{\prime} \cdot L^{\prime}(f)}, \quad \phi_{5}(f)=\frac{2 \cdot \pi \cdot f}{R^{\prime}(f) / L^{\prime}(f)+G^{\prime} / C^{\prime}}=\frac{\phi_{1}(f)}{1+1 / \phi_{4}(f)} .
\end{gathered}
$$

From the numerical data associated with the monotonic functions in Figures 13, 14, and 16 it is obtained $\phi_{1}(110.9 \mathrm{KHz}) \approx 1, \phi_{1}(1289.9 \mathrm{KHz}) \approx 10, \phi_{1}(10 \mathrm{MHz}) \approx 32.653 \gg 1$, $\phi_{2}(110.9 \mathrm{KHz}) \approx 13.943 \cdot 10^{9}$ and $\phi_{4}(0) \approx 1.391 \cdot 10^{10}$. Herefrom and from (2.15) it follows 


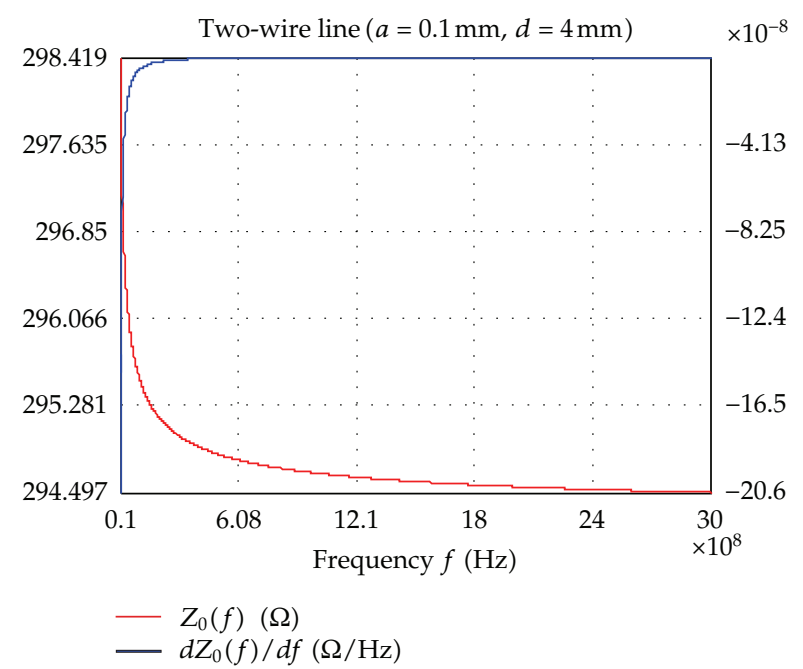

Figure 11: $Z_{0}(f)$ and $d Z_{0}(f) / d f$ for the considered two-wire line.

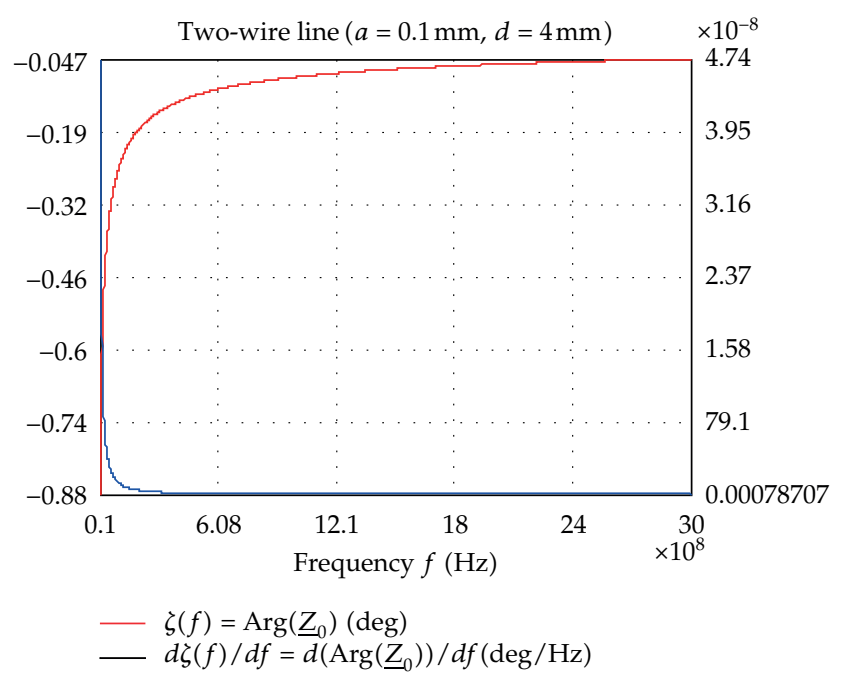

Figure 12: $\zeta(f)$ and $d \zeta(f) / d f$ for the considered two-wire line.

$\phi_{1}(f) \approx \phi_{5}(f)$. Since in this case the Heaviside's condition [7] [ $\left.\Leftrightarrow \phi_{4}(f)=1\right]$ is not satisfied, distortionless transmission is not possible. Another two functions, $\Lambda(f)=|\Gamma(j \cdot 2 \pi \cdot f)|$ and $\vartheta(f)=\operatorname{Arg}[\Gamma(j \cdot 2 \pi \cdot f)][" \Gamma$ " are the propagation function, see (A.10) in Appendix], also, play important role in analysis and they are depicted in Figures 17 and 18, respectively, in range $f \in[0.01,3][\mathrm{GHz}]$. From data associated with these functions it is obtained: $\Lambda(10 \mathrm{MHz}) \approx$ $0.319, \Lambda(3 \mathrm{GHz}) \approx 94.598, \vartheta(10 \mathrm{MHz}) \approx 89.123[\mathrm{deg}]$ and $\vartheta(3 \mathrm{GHz}) \approx 89.953[\mathrm{deg}]$.

Since $\Gamma(j \cdot 2 \pi \cdot f)=\sqrt{\left[R^{\prime}(f)+j \cdot 2 \pi \cdot f \cdot L^{\prime}(f)\right] \cdot\left(G^{\prime}+j \cdot 2 \pi \cdot f \cdot C^{\prime}\right)}=\Lambda(f) \cdot \exp [j \cdot$ $\vartheta(f)]$ and since in the range $f \in[0.01,3][\mathrm{GHz}]$ it holds: $\phi_{1}(f) \approx \phi_{5}(f) \gg 1, \phi_{2}(f) \gg 1$, $\Lambda(f) \in[0.319,94.598]$ and $\vartheta(f) \in[89.123,89.953][\mathrm{deg}]$, then: $\Lambda(f) \approx 2 \pi \cdot f \cdot\left[L^{\prime}(f) \cdot C^{\prime}\right]^{1 / 2}=$ $1 / \phi_{3}(f), \vartheta(f)=\pi / 2-\chi(f)[0<\chi(f)<\pi / 200]$ and $\Gamma(j \cdot 2 \pi \cdot f)=\Lambda(f) \cdot \exp (j \cdot \pi / 2) \cdot$ $\exp [-j \cdot x(f)]=j \cdot \Lambda(f) \cdot\{\cos [x(f)]-j \cdot \sin [x(f)]\}=\{\sin [x(f)]+j \cdot \cos [x(f)]\} / \phi_{3}(f)$, where the deviation angle $x(f)=\pi / 2-\vartheta(f)$ can be approximated (The percentage error of this 


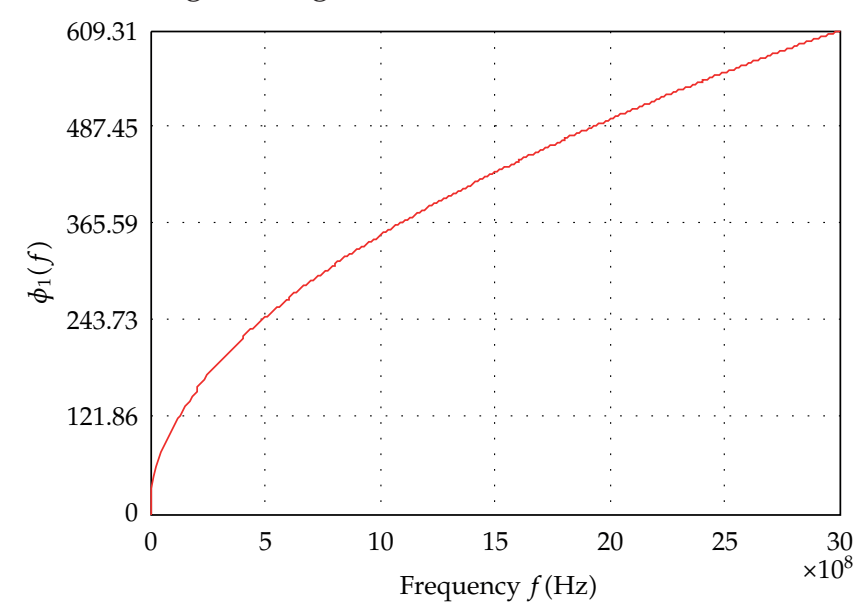

Figure 13: A dimensionless parameter $\phi_{1}(f)$ of two-wire line.

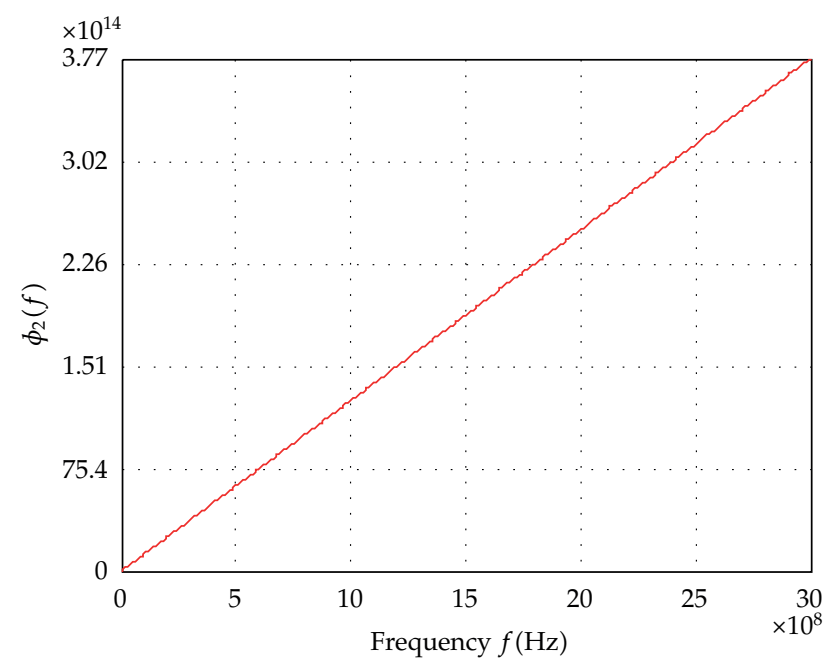

Figure 14: A dimensionless parameter $\phi_{2}(f)$ of two-wire line.

approximation is positive and $<0.032 \%$ in the entire frequency range $f \in[0.01,3][\mathrm{GHz}]$.) with,

$$
\begin{aligned}
x(f) & =\frac{\pi}{2}-\vartheta(f)=\frac{\pi}{2}-\frac{1}{2} \cdot a \tan \left(2 \pi \cdot f \cdot \frac{L^{\prime}(f) / R^{\prime}(f)+C^{\prime} / G^{\prime}}{1-4 \cdot \pi^{2} \cdot f^{2}\left(\left(L^{\prime}(f) \cdot C^{\prime}\right) /\left(R^{\prime}(f) \cdot G^{\prime}\right)\right)}\right) \\
& \approx \frac{1}{2} \cdot a \tan \left(\frac{R^{\prime}(f) / L^{\prime}(f)+G^{\prime} / C^{\prime}}{2 \pi \cdot f}\right) \approx \frac{R^{\prime}(f) / L^{\prime}(f)+G^{\prime} / C^{\prime}}{4 \pi \cdot f} .
\end{aligned}
$$

For the transmission line with length $\ell$ let us define the functions: $\varepsilon(\omega)=x(\omega / 2 \pi)=$ $\pi / 2-\vartheta(\omega / 2 \pi)$ and $\theta(j \cdot \omega)=\Gamma(j \cdot \omega) \cdot(\ell-x)=A(\omega, x) \cdot \exp [j \cdot \vartheta(\omega / 2 \pi)]=A(\omega, x)$. $\{\sin [\varepsilon(\omega)]+j \cdot \cos [\varepsilon(\omega)]\}\{x \in[0, \ell]\}$, where $A(\omega, x)=|\Gamma(j \cdot \omega)| \cdot(\ell-x)=\Lambda(\omega / 2 \pi) \cdot(\ell-x)$. For this line, in frequency range $f \in[0.01,3][\mathrm{GHz}]$ we have $A(\omega, x) \approx \omega \cdot\left[L^{\prime}(\omega / 2 \pi) \cdot C^{\prime}\right]^{1 / 2}$. $(\ell-x)=(\ell-x) / \phi_{3}(\omega / 2 \pi)$ and $0<\varepsilon(\omega)<\pi / 200$-whereby $\theta(j \cdot \omega)$ becomes almost pure imaginary number. We could have obtained this result in a different way. To se that, let us 


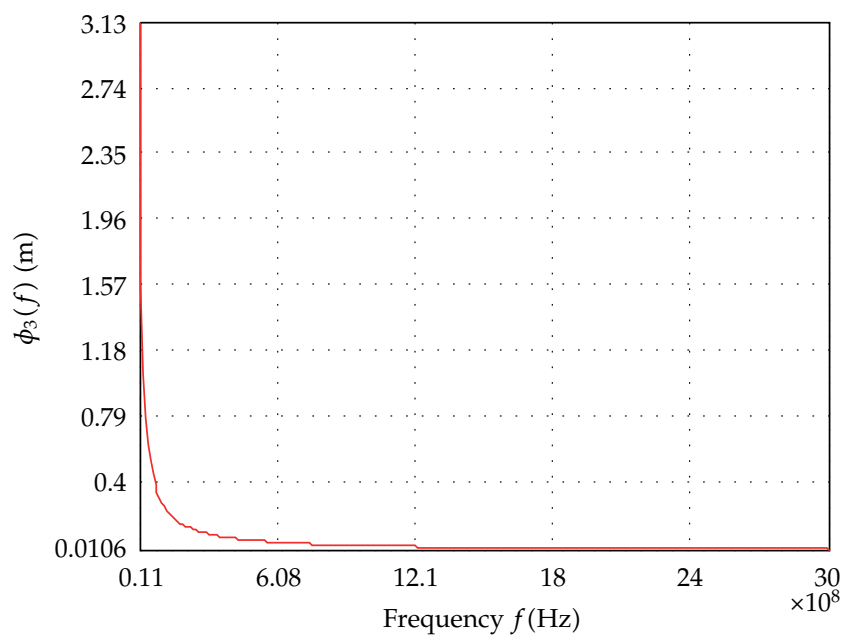

Figure 15: Parameter $\phi_{3}(f)$ of two-wire line.

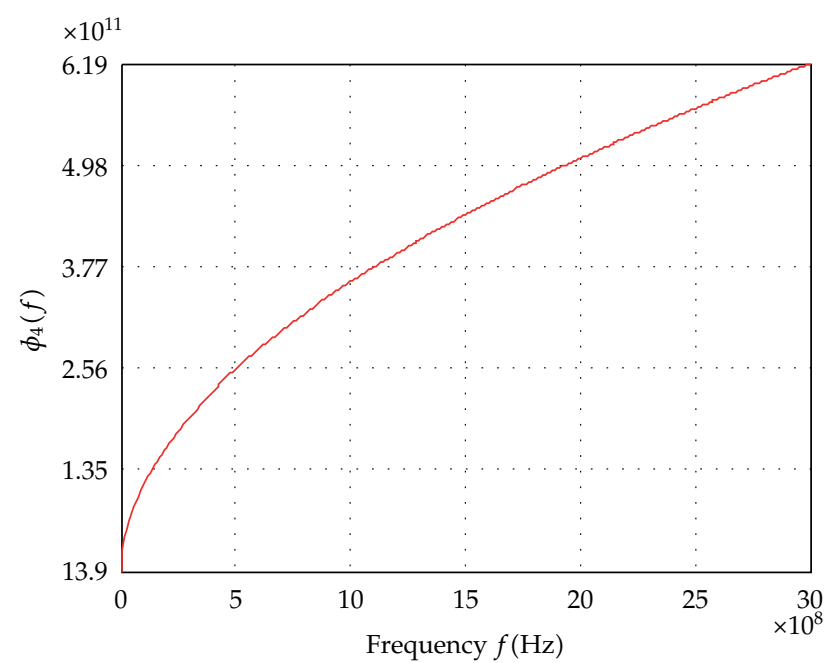

Figure 16: A dimensionless parameter $\phi_{4}(f)$ of two-wire line.

write $\Gamma(j \cdot 2 \pi \cdot f)=\sqrt{\left[R^{\prime}(f)+j \cdot 2 \pi \cdot f \cdot L^{\prime}(f)\right] \cdot\left(G^{\prime}+j \cdot 2 \pi \cdot f \cdot C^{\prime}\right)}=a(f)+j \cdot b(f)$, where it holds,

$$
\begin{aligned}
& a(f)=\sqrt{\frac{1}{2} \cdot L^{\prime}(f) \cdot C^{\prime}\left\{\sqrt{\left[(2 \pi \cdot f)^{2}+\frac{R^{\prime}(f)^{2}}{L^{\prime}(f)^{2}}\right] \cdot\left[\left((2 \pi \cdot f)^{2}+\frac{G^{\prime 2}}{C^{\prime 2}}\right)\right]}+\frac{R^{\prime}(f)}{L^{\prime}(f)} \cdot \frac{G^{\prime}}{C^{\prime}}-(2 \pi \cdot f)^{2}\right\}} \\
& b(f)=\sqrt{\frac{1}{2} \cdot L^{\prime}(f) \cdot C^{\prime}\left\{\sqrt{\left.\left[(2 \pi \cdot f)^{2}+\frac{R^{\prime}(f)^{2}}{L^{\prime}(f)^{2}}\right] \cdot\left[(2 \pi \cdot f)^{2}+\frac{G^{\prime 2}}{C^{\prime 2}}\right)\right]}-\frac{R^{\prime}(f)}{L^{\prime}(f)} \cdot \frac{G^{\prime}}{C^{\prime}}+(2 \pi \cdot f)^{2}\right\}} \\
& \text { phase "constant". }
\end{aligned}
$$




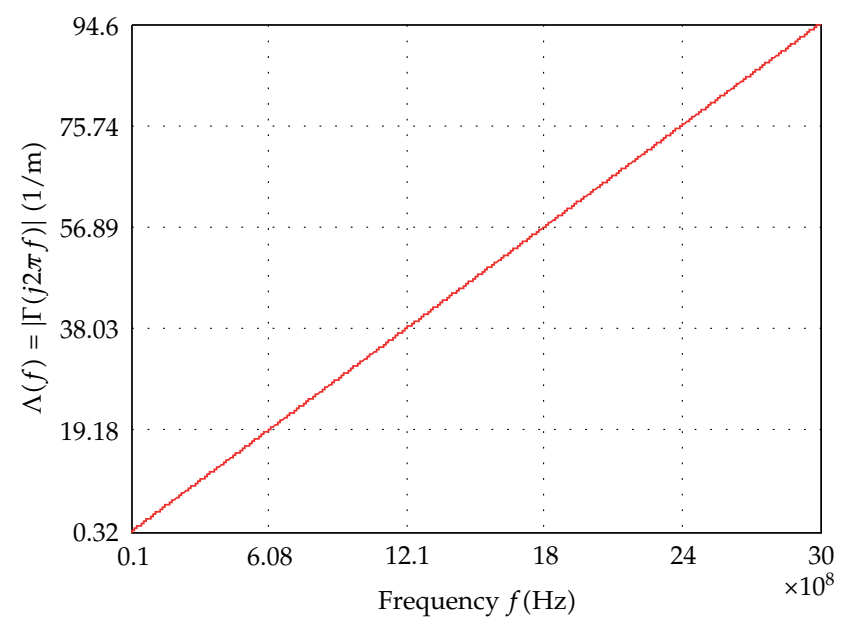

Figure 17: Magnitude of propagation function (two-wire line).

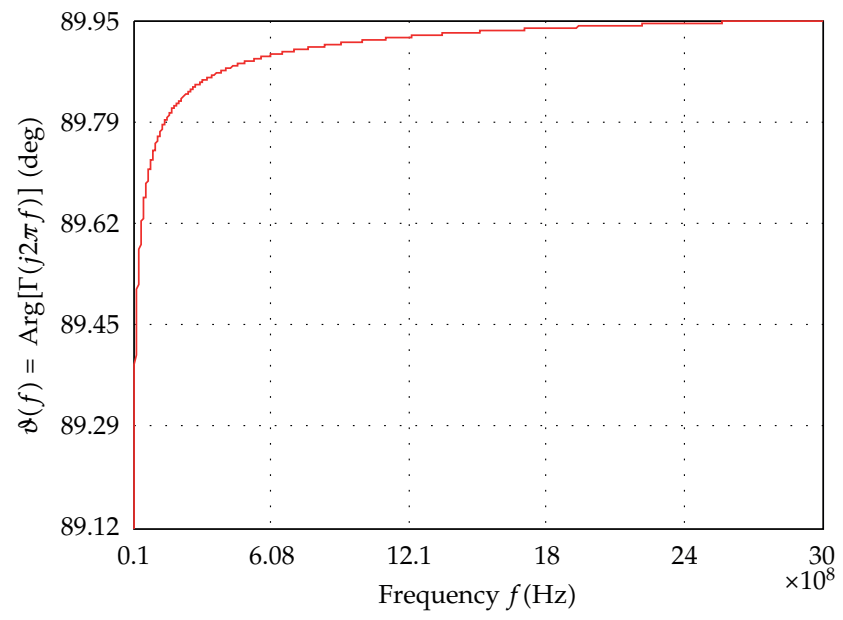

Figure 18: Argument of propagation function (two-wire line).

The previous two functions are depicted in Figures 19 and 20 in the frequency range $f \in[0,3]$ [GHz].

From relations (2.17) we obtain the approximations $a a(f)$ and $a b(f)$ of $a(f)$ and $b(f)$, respectively, in the frequency range $f \in[0.01,3][\mathrm{GHz}]$, since there it holds $\phi_{1}(f) \gg 1$ and $\phi_{2}(f) \gg 1$,

$$
\begin{aligned}
& a a(f) \approx \frac{1}{2}\left[R^{\prime}(f) \cdot \sqrt{\frac{C^{\prime}}{L^{\prime}(f)}}+G^{\prime} \cdot \sqrt{\frac{L^{\prime}(f)}{C^{\prime}}}\right], \\
& a b(f) \approx \sqrt{L^{\prime}(f) \cdot C^{\prime}(f)}\left\{2 \pi \cdot f+\frac{1}{16 \pi \cdot f} \cdot\left[\frac{R^{\prime}(f)}{L^{\prime}(f)}-\frac{G^{\prime}}{C^{\prime}}\right]^{2}\right\}
\end{aligned}
$$




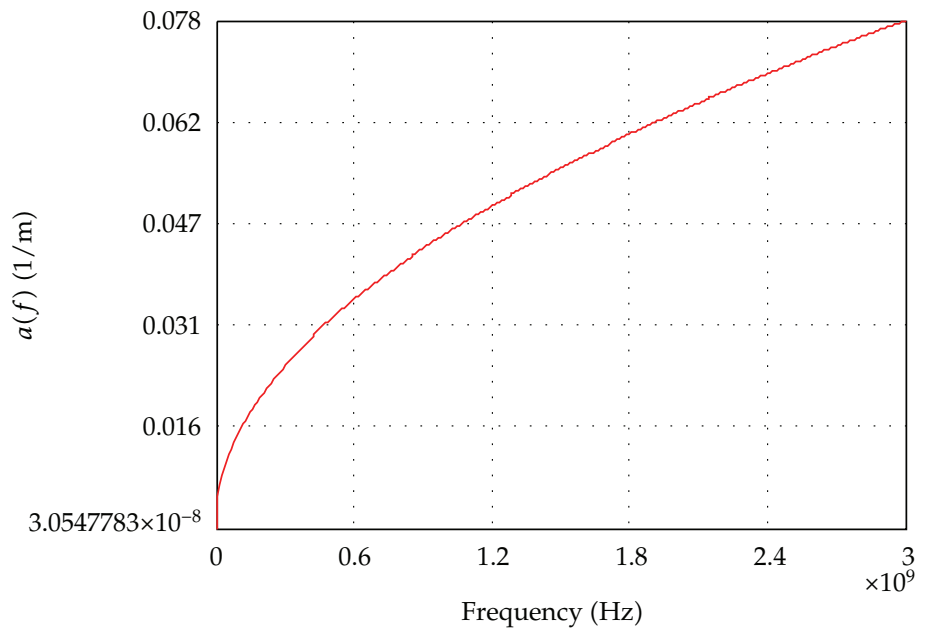

Figure 19: The attenuation "constant" of two-wire line.

and, also, we have, $\quad Z_{0}(j \cdot 2 \pi \cdot f)=\sqrt{\frac{R^{\prime}(f)+j \cdot 2 \pi \cdot f \cdot L^{\prime}(f)}{G^{\prime}+j \cdot 2 \pi \cdot f \cdot C^{\prime}}}$

$$
\approx \sqrt{\frac{L^{\prime}(f)}{C^{\prime}}} \cdot\left\{1-j \cdot \frac{1}{4 \pi \cdot f}\left[\frac{R^{\prime}(f)}{L^{\prime}(f)}-\frac{G^{\prime}}{C^{\prime}}\right]\right\} .
$$

The functions $a a(f)$ and $a b(f)$ are depicted in Figures 21 and 22, respectively, in the frequency range $f \in[0.01,3][\mathrm{GHz}]$, where we have as previously that it holds $\Gamma(j \cdot \omega) \approx$ $a a(\omega / 2 \pi)+j \cdot a b(\omega / 2 \pi) \approx\{\sin [\varepsilon(\omega)]+j \cdot \cos [\varepsilon(\omega)]\} / \phi_{3}(\omega / 2 \pi)$, as it has been expected.

We will now emphasize the importance of function $\theta=\theta(s, x)=\Gamma(s) \cdot(\ell-x)\{x \in[0, \ell]\}$ in the following.

(a) Constituting of functions $\sinh (\theta) / \theta$ and $\tanh (\theta / 2) /(\theta / 2)$ that play fundamental role in producing uniform three-terminal networks nominally equivalent to short-line segments [7] and in realization of these networks in the specified frequency range $\left(f_{0}-B / 2, f_{0}+\right.$ $B / 2$ ) by approximately equivalent three-terminal lumped $R L C$ networks. The purpose of this approach is to involve the application of PSPICE, so as to facilitate the steady-state analysis of transmission lines with arbitrary terminations and band-limited signals, instead of solving the pair of so-called telegraph equations, hyperbolic, linear, partial diffrential equations obtained from relations (A.4) in the Appendix,

$$
\begin{aligned}
& \frac{\partial^{2} u(t, x)}{\partial x^{2}}=L^{\prime} \cdot C^{\prime} \cdot \frac{\partial^{2} u(t, x)}{\partial t^{2}}+\left(L^{\prime} \cdot G^{\prime}+C^{\prime} \cdot R^{\prime}\right) \cdot \frac{\partial u(t, x)}{\partial t}+R^{\prime} \cdot G^{\prime} \cdot u(t, x), \\
& \frac{\partial^{2} i(t, x)}{\partial x^{2}}=L^{\prime} \cdot C^{\prime} \cdot \frac{\partial^{2} i(t, x)}{\partial t^{2}}+\left(L^{\prime} \cdot G^{\prime}+C^{\prime} \cdot R^{\prime}\right) \cdot \frac{\partial i(t, x)}{\partial t}+R^{\prime} \cdot G^{\prime} \cdot i(t, x) .
\end{aligned}
$$

To alternatively determine the voltage and current variations in time at any place on the finite length line, we may firstly perform the Fourier analysis of excitation signal and retain a reasonable number of its spectral components, then determine their transfer one at a time 


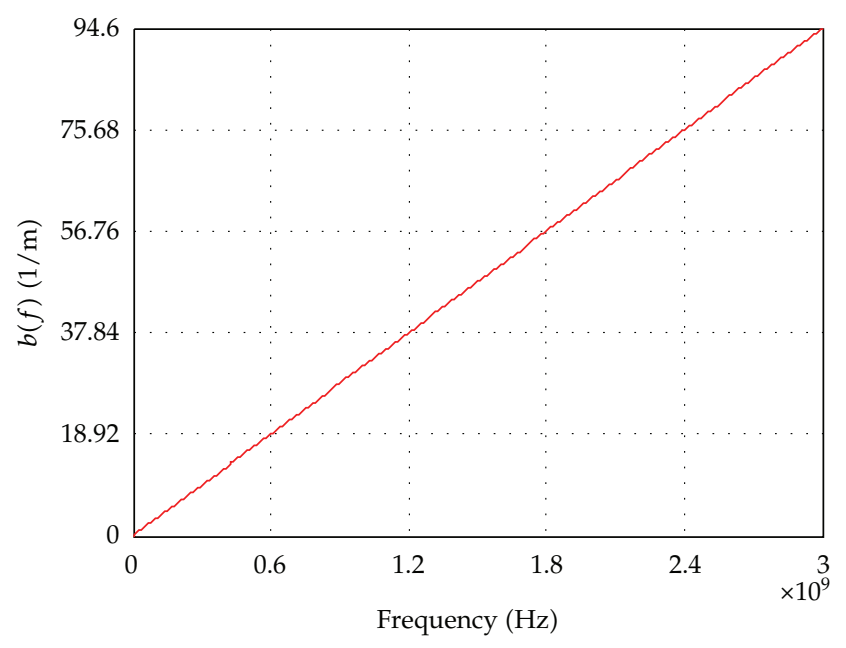

Figure 20: The phase "constant" of two-wire line.

to the specified place on the transmission line by using (A.17) from the Appendix and finally synthesize the overall response by superposition of the obtained single-frequency responses.

(b) Calculation of $M(s, x), N(s, x), U(s, x)$, and $I(s, x)$ from (A.17) and $Z(s, x)$ from (A.18), in general, and for the finite length open-circuited line $\left[Z_{L}(s) \rightarrow \infty\right]$, in particular, by using expansions:

$$
\begin{gathered}
M(s, x)=\frac{U(s, x)}{U(s, 0)}=\frac{\prod_{n=1}^{\infty}\left\{1+[(2 \Gamma \cdot(\ell-x)) /((2 n-1) \cdot \pi)]^{2}\right\}}{\prod_{n=1}^{\infty}\left\{1+[(2 \Gamma \cdot \ell) /((2 n-1) \cdot \pi)]^{2}\right\}}, \\
N(s, x)=\frac{I(s, x)}{I(s, 0)}=\frac{\ell-x}{\ell} \cdot \frac{\prod_{n=1}^{\infty}\left\{1+[(\Gamma \cdot(\ell-x)) /(n \cdot \pi)]^{2}\right\}}{\prod_{n=1}^{\infty}\left[1+((\Gamma \cdot \ell) /(n \cdot \pi))^{2}\right]}, \\
Z(s, x)=\frac{U(s, x)}{I(s, x)}=\frac{\prod_{n=1}^{\infty}\left\{1+[(2 \Gamma \cdot(\ell-x)) /((2 n-1) \cdot \pi)]^{2}\right\}}{\Gamma \cdot(\ell-x) \cdot \prod_{n=1}^{\infty}\left\{1+[(\Gamma \cdot(\ell-x)) /(n \cdot \pi)]^{2}\right\}} \cdot Z_{0} \quad\left[Z_{L}(s) \longrightarrow \infty\right],
\end{gathered}
$$

thus placing into evidence the pole-zero location of $M(s, x), N(s, x)$, and $Z(s, x)$.

The relations (2.20) are produced by using Weierstass's factor expansions [8] of transcendental functions appearing in (A.17) and (A.18) into infinite product forms,

$$
\frac{\sinh (\theta)}{\theta}=\prod_{n=1}^{\infty}\left(1+\frac{\theta^{2}}{n^{2} \cdot \pi^{2}}\right), \quad \cosh (\theta)=\prod_{n=1}^{\infty}\left[1+\frac{4 \cdot \theta^{2}}{(2 n-1)^{2} \cdot \pi^{2}}\right]
$$

For the open-circuited two-wire line with length $\ell=0.1[\mathrm{~m}]$ in Figures $23 \div 26$ are depicted for $x \in[0,0.1][\mathrm{m}]$ and $f \in[0,3][\mathrm{GHz}]$ the variations of $|M(j \cdot 2 \pi \cdot f, x)|$, $\operatorname{Arg}[M(j \cdot 2 \pi \cdot f, x)][\mathrm{deg}],|N(j \cdot 2 \pi \cdot f, x)|$ and $\operatorname{Arg}[N(j \cdot 2 \pi \cdot f, x)]$ [deg], respectively, on the $\operatorname{grid}(x) \times \operatorname{grid}(f)=50 \times 60$. In Figures 23 and 25 we observe the presence of voltage and current resonances at different places on the line, as is it might be expected from (2.20), 


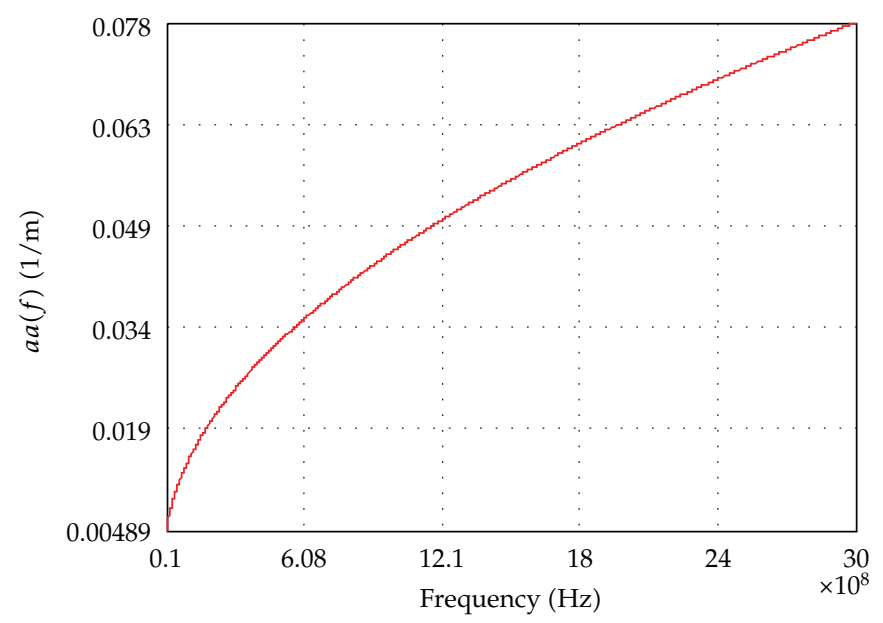

Figure 21: Approximation of the attenuation "constant".

at six discrete frequencies altogether, in the two disjoint sets. Also, we may notice in Figures 24 and 26 that variations of Argfunctions are very complex with abrupt transitions. For the line terminated in $Z_{L}(s)$ the diagrams analogous to those in Figures $23 \div 26$ could also be drawn easily, provided that the impedance $Z_{0}(s)$ is taken into account [see relation (A.17)].

When the line is sufficiently short, some approximations can be made leading to satisfactory results without need to cope with the cumulative products (2.21). To see that, suppose that line length is $\ell \leq \ell_{0}<\pi /\left\{2 \cdot \mid\left[\left.\Gamma(j \cdot 2 \pi \cdot f)\right|_{\max }\right\} \approx 16.6[\mathrm{~mm}]\left\{\Leftrightarrow|\theta|_{\max }<\pi / 2\right\}\right.$ and assume, say, $\ell_{0}=16[\mathrm{~mm}]$. Recall that $=\theta(j \cdot \omega, x)=\Gamma(j \cdot \omega) \cdot(\ell-x)=|\theta(j \cdot \omega, x)| \cdot \exp \{j \cdot$ $\arg [\Gamma(j \cdot \omega)]\}\{x \in[0, \ell]\}$, then take (2.21) and write

$$
\begin{aligned}
\frac{\sinh (\theta)}{\theta}= & \prod_{n=1}^{\infty}\left(1+\frac{\theta^{2}}{n^{2} \cdot \pi^{2}}\right)=\exp \left[\sum_{n=1}^{\infty} \ln \left(1+\frac{\theta^{2}}{n^{2} \cdot \pi^{2}}\right)\right] \\
= & {\left[\exp \frac{\theta^{2}}{\pi^{2}} \cdot \sum_{n=1}^{\infty} \frac{1}{n^{2}}-\frac{\theta^{4}}{2 \cdot \pi^{4}} \cdot \sum_{n=1}^{\infty} \frac{1}{n^{4}}+\frac{\theta^{6}}{3 \cdot \pi^{6}} \cdot \sum_{n=1}^{\infty} \frac{1}{n^{6}}-\frac{\theta^{8}}{4 \cdot \pi^{8}} \cdot \sum_{n=1}^{\infty} \frac{1}{n^{8}}\right.} \\
& \left.+\frac{\theta^{10}}{5 \cdot \pi^{10}} \cdot \sum_{n=1}^{\infty} \frac{1}{n^{10}} \pm \ldots\right] \\
= & \exp \left(\frac{\theta^{2}}{6}-\frac{\theta^{4}}{180}+\frac{\theta^{6}}{2835}-\frac{\theta^{8}}{37800}+\frac{\theta^{10}}{467775} \pm \cdots\right), \\
\cosh (\theta)= & \prod_{n=1}^{\infty}\left[1+\frac{4 \cdot \theta^{2}}{(2 n-1)^{2} \cdot \pi^{2}}\right]=\exp \left\{\sum_{n=1}^{\infty} \ln \left[1+\frac{4 \cdot \theta^{2}}{(2 n-1)^{2} \cdot \pi^{2}}\right]\right\} \\
= & {\left[\exp \frac{4 \cdot \theta^{2}}{\pi^{2}} \cdot \sum_{n=1}^{\infty} \frac{1}{(2 n-1)^{2}}-\frac{8 \cdot \theta^{4}}{\pi^{4}} \cdot \sum_{n=1}^{\infty} \frac{1}{(2 n-1)^{4}}+\frac{64 \cdot \theta^{6}}{3 \cdot \pi^{6}} \cdot \sum_{n=1}^{\infty} \frac{1}{(2 n-1)^{6}}\right.} \\
& \left.\quad-\frac{64 \cdot \theta^{8}}{\pi^{8}} \cdot \sum_{n=1}^{\infty} \frac{1}{(2 n-1)^{8}}+\frac{1024 \cdot \theta^{10}}{5 \cdot \pi^{10}} \cdot \sum_{n=1}^{\infty} \frac{1}{(2 n-1)^{10}} \pm \cdots\right] \\
= & \exp \left(\frac{\theta^{2}}{2}-\frac{\theta^{4}}{12}+\frac{\theta^{6}}{45}-\frac{17 \cdot \theta^{8}}{2520}+\frac{31 \cdot \theta^{10}}{14175} \pm \cdots\right) .
\end{aligned}
$$




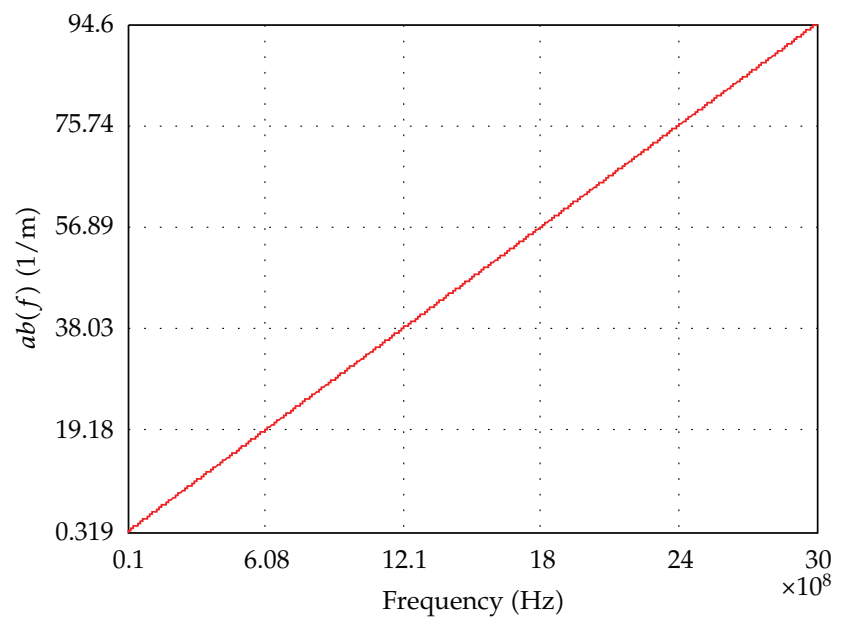

Figure 22: Approximation of the phase "constant".

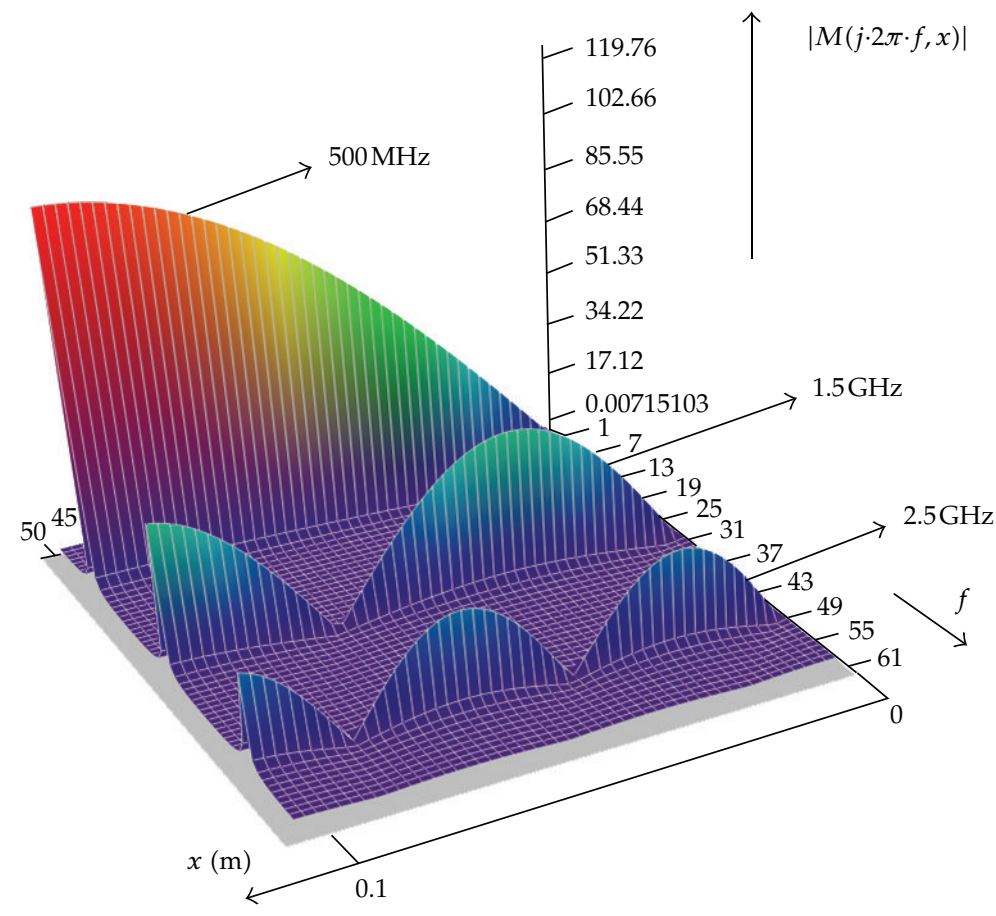

Figure 23: The magnitude of the voltage transmittance for two-wire line $(\ell=0.1[\mathrm{~m}])$ in frequency range $f \in[0,3][\mathrm{GHz}]$.

The following finite sums of infinite series [9] have been exploited in (2.22),

$$
\begin{gathered}
A_{2 p}=\sum_{n=1}^{\infty} \frac{1}{n^{2 p}}(\overline{p=1,5}), \quad A_{2}=\frac{\pi^{2}}{6}, \quad A_{4}=\frac{\pi^{4}}{90}, \quad A_{6}=\frac{\pi^{6}}{945}, \quad A_{8}=\frac{\pi^{8}}{9450}, \\
A_{10}=\frac{5 \cdot 2^{8} \cdot \pi^{10}}{33 \cdot 10 !},
\end{gathered}
$$




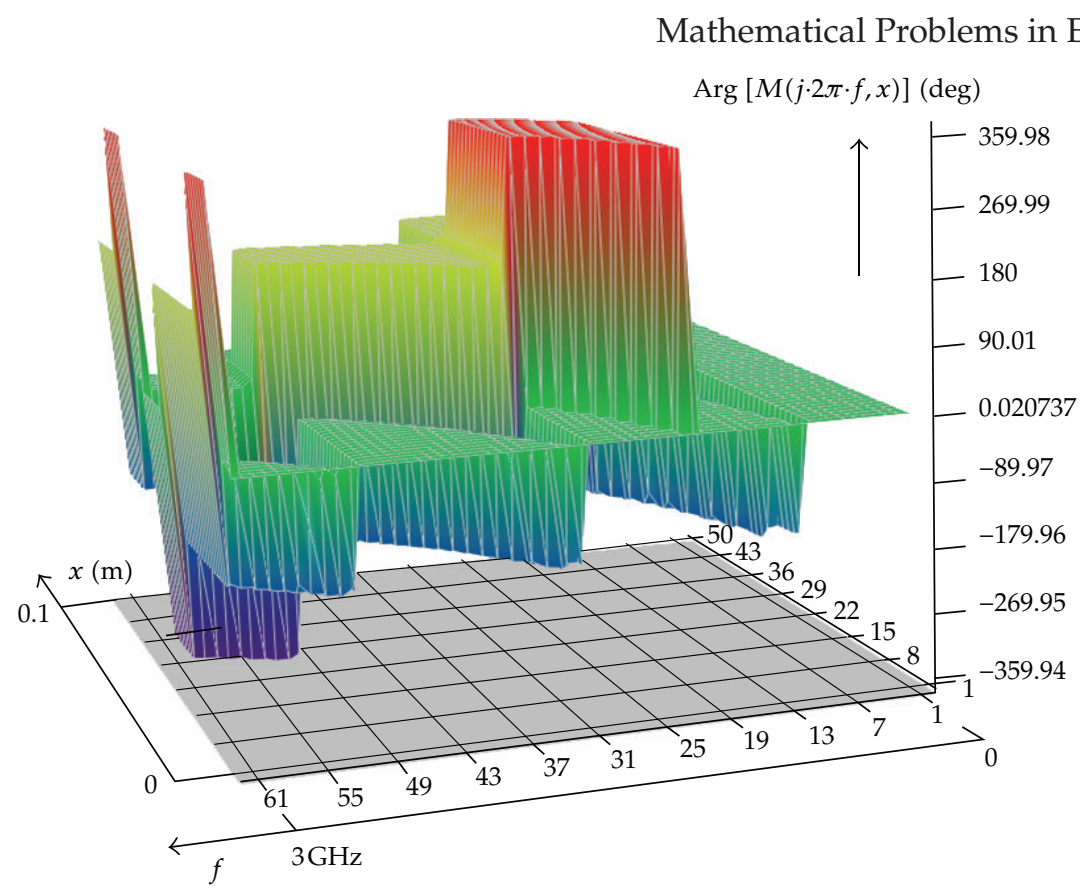

Figure 24: The argument of the voltage transmittance for two-wire line $(\ell=0.1[\mathrm{~m}])$ in frequency range $f \in[0,3][\mathrm{GHz}]$.

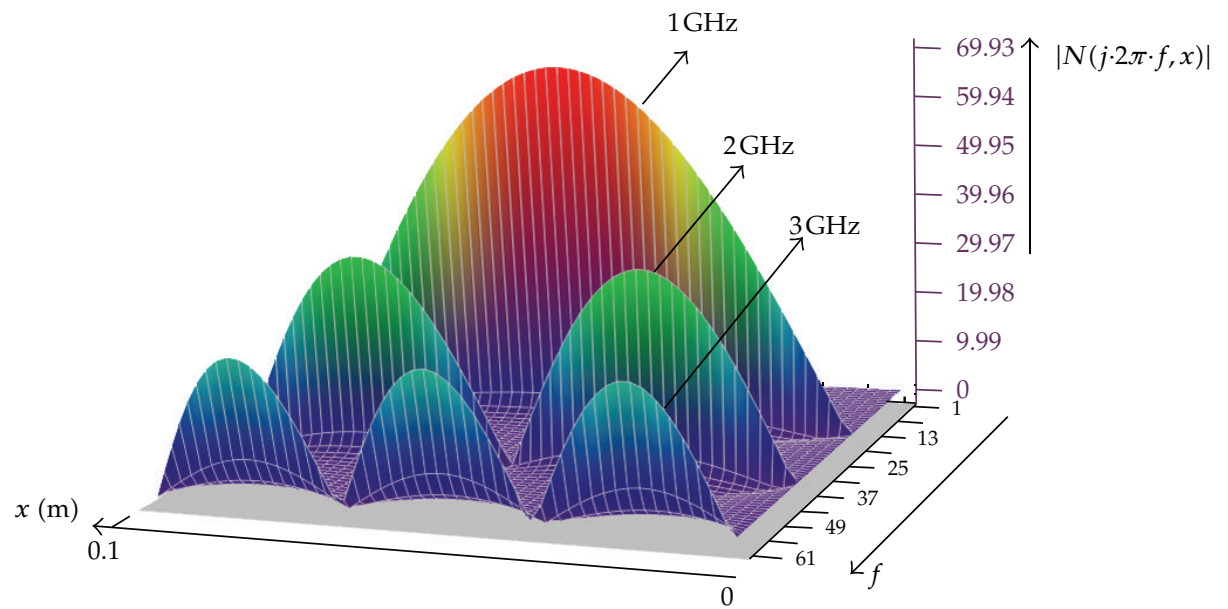

Figure 25: The magnitude of the current transmittance for two-wire line $(\ell=0.1[\mathrm{~m}])$ in frequency range $f \in[0,3][\mathrm{GHz}]$.

$$
\begin{aligned}
B_{2 p}=\sum_{n=1}^{\infty} \frac{1}{(2 n-1)^{2 p}}(\overline{p=1,5}), \quad B_{2}=\frac{\pi^{2}}{8}, \quad B_{4}=\frac{\pi^{4}}{96}, \quad B_{6}=\frac{\pi^{6}}{960}, \quad B_{8}=\frac{17 \cdot \pi^{8}}{161280}, \\
B_{10}=\frac{31 \cdot \pi^{10}}{35 \cdot 81 \cdot 2^{10}} .
\end{aligned}
$$

The infinite complex series (2.22) in almost pure imaginary $\theta$ are convergent for $\ell \leq$ $\ell_{0}, x \in[0, \ell]$ and $f \in[0,3][\mathrm{GHz}]$. If $\ell$ is sufficiently less than $\ell_{0}$ and $x \in[0, \ell]$, then by 


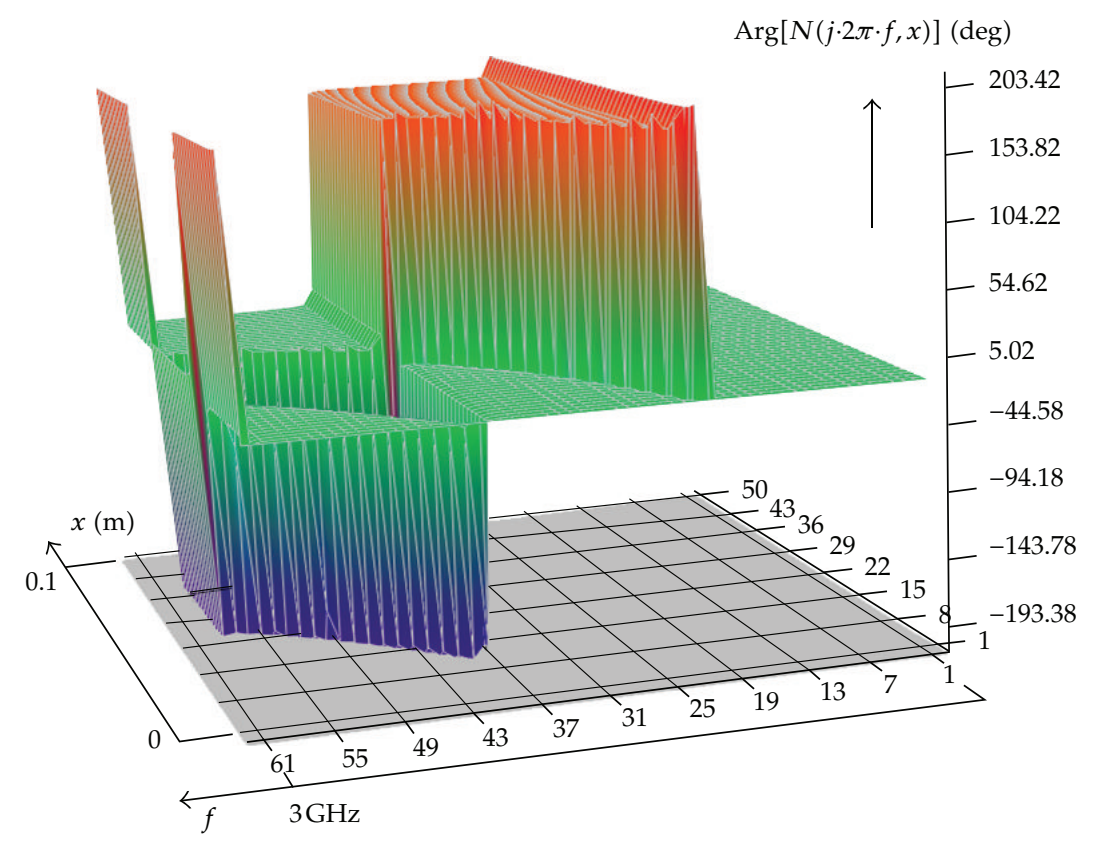

Figure 26: The argument of the current transmittance for two-wire line $(\ell=0.1[\mathrm{~m}])$ in frequency range $f \in[0,3][\mathrm{GHz}]$.

retaining only the first five $\theta$ terms in (2.22) the following small-error approximations are produced

$$
\begin{gathered}
\sinh (\theta) \approx \sinh _{A}(\theta)=\theta \cdot \exp \left(\frac{\theta^{2}}{6}-\frac{\theta^{4}}{180}+\frac{\theta^{6}}{2835}-\frac{\theta^{8}}{37800}+\frac{\theta^{10}}{467775}\right), \\
\cosh (\theta) \approx \cosh _{A}(\theta)=\exp \left(\frac{\theta^{2}}{2}-\frac{\theta^{4}}{12}+\frac{\theta^{6}}{45}-\frac{17 \cdot \theta^{8}}{2520}+\frac{31 \cdot \theta^{10}}{14175}\right) .
\end{gathered}
$$

For the open-circuited two-wire line with $\ell=0.01[\mathrm{~m}]$, in Figures 27, 28, 29, and 30 they are depicted on $\operatorname{grid}(x) \times \operatorname{grid}(f)=40 \times 60$ in the range $f \in[0,3][\mathrm{GHz}]$ and range $x \in[0,0.01][\mathrm{m}]$, respectively:

(i) the voltage-transmittance magnitude approximation percentage error:

$\mathrm{ER}_{1}(f, x)=\left\{\mid\left[\cosh _{A} \theta(j \cdot 2 \pi \cdot f, x) / \cosh _{A}(j \cdot 2 \pi \cdot f, 0)\right] /[\cosh \theta(j \cdot 2 \pi \cdot f, x) / \cosh (j\right.$ $2 \pi \cdot f, 0)] \mid-1\} \cdot 100$;

(ii) the voltage-transmittance phase approximation absolute error:

$\mathrm{ER}_{2}(f, x)=\arg \left[\cosh _{A} \theta(j \cdot 2 \pi \cdot f, x) \cdot \cosh (j \cdot 2 \pi \cdot f, 0) / \cosh _{A}(j \cdot 2 \pi \cdot f, 0) \cdot \cosh \theta(j \cdot\right.$ $2 \pi \cdot f, x)]$;

(iii) the current-transmittance magnitude approximation percentage error:

$\mathrm{ER}_{3}(f, x)=\left\{\mid\left[\sinh _{A} \theta(j \cdot 2 \pi \cdot f, x) / \sinh _{A}(j \cdot 2 \pi \cdot f, 0)\right] /[\sinh \theta(j \cdot 2 \pi \cdot f, x) / \sinh (j \cdot\right.$ $2 \pi \cdot f, 0)] \mid-1\} \cdot 100$;

(iv) the current-transmittance phase approximation absolute error:

$\mathrm{ER}_{4}(f, x)=\arg \left[\sinh _{A} \theta(j \cdot 2 \pi \cdot f, x) \cdot \sinh (j \cdot 2 \pi \cdot f, 0) / \sinh _{A}(j \cdot 2 \pi \cdot f, 0) \cdot \sinh \theta(j \cdot\right.$ $2 \pi \cdot f, x)]$. 


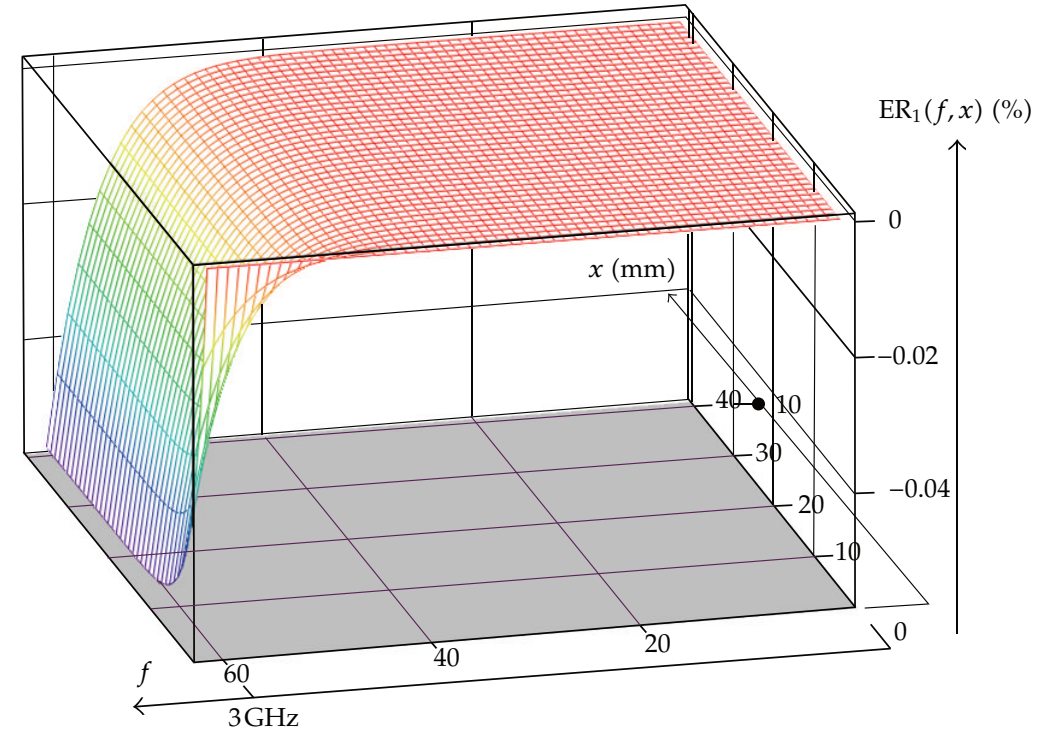

Figure 27: Voltage-transmittance magnitude approximation percentage error $\operatorname{ER}_{1}(f, x)$.

It can be observed in Figures $27 \div 30$ that in the given range of $f$ and $x$, the errors $\mathrm{ER}_{1}$ and $E R_{3}$ are negative $\left(\left|E R_{1}\right|<0.06 \%\right.$ and $\left.\left|E R_{3}\right|<10^{-5} \%\right)$, whereas the errors $E R_{2}$ and $E R_{4}$ are positive $\left(\mathrm{ER}_{2}<3.36 \cdot 10^{-4}[\mathrm{deg}]\right.$ and $\left.\mathrm{ER}_{4}<5.75 \cdot 10^{-8}[\mathrm{deg}]\right)$. The upper limit of $\left|\mathrm{ER}_{3}\right|$ is lower than of $\left|E R_{1}\right|$ and the upper limit of $E_{4}$ is lower than of $E_{2}$.

If $|\theta|$ is sufficiently small $(|\theta| \ll 1)$, from (2.24) further approximations are obtained:

(i)

$$
\begin{aligned}
\sinh (\theta) & \approx \sinh _{A}(\theta) \\
& =\theta \cdot \exp \left(\frac{\theta^{2}}{6}-\frac{\theta^{4}}{180}+\frac{\theta^{6}}{2835}-\frac{\theta^{8}}{37800}+\frac{\theta^{10}}{467775}\right) \\
& \approx \theta \cdot\left(1+\frac{\theta^{2}}{6}-\frac{\theta^{4}}{180}+\frac{\theta^{6}}{2835}-\frac{\theta^{8}}{37800}+\frac{\theta^{10}}{467775}\right) \\
& =\theta+\frac{\theta^{3}}{6}-\frac{\theta^{5}}{180}+\frac{\theta^{7}}{2835}-\frac{\theta^{9}}{37800}+\frac{\theta^{11}}{467775} \\
& \approx \theta+\frac{\theta^{3}}{6}
\end{aligned}
$$

which partly resembles to Maclaurin's expansion of $\sinh (\theta)$, that is, $\sinh (\theta)=\theta+$ $\theta^{3} / 3 !+\theta^{5} / 5 !+\ldots$,

(ii)

$$
\begin{aligned}
\cosh (\theta) & \approx \cosh _{A}(\theta)=\exp \left(\frac{\theta^{2}}{2}-\frac{\theta^{4}}{12}+\frac{\theta^{6}}{45}-\frac{17 \cdot \theta^{8}}{2520}+\frac{31 \cdot \theta^{10}}{14175}\right) \\
& \approx 1+\frac{\theta^{2}}{2}-\frac{\theta^{4}}{12}+\frac{\theta^{6}}{45}-\frac{17 \cdot \theta^{8}}{2520}+\frac{31 \cdot \theta^{10}}{14175} \approx 1+\frac{\theta^{2}}{2}
\end{aligned}
$$




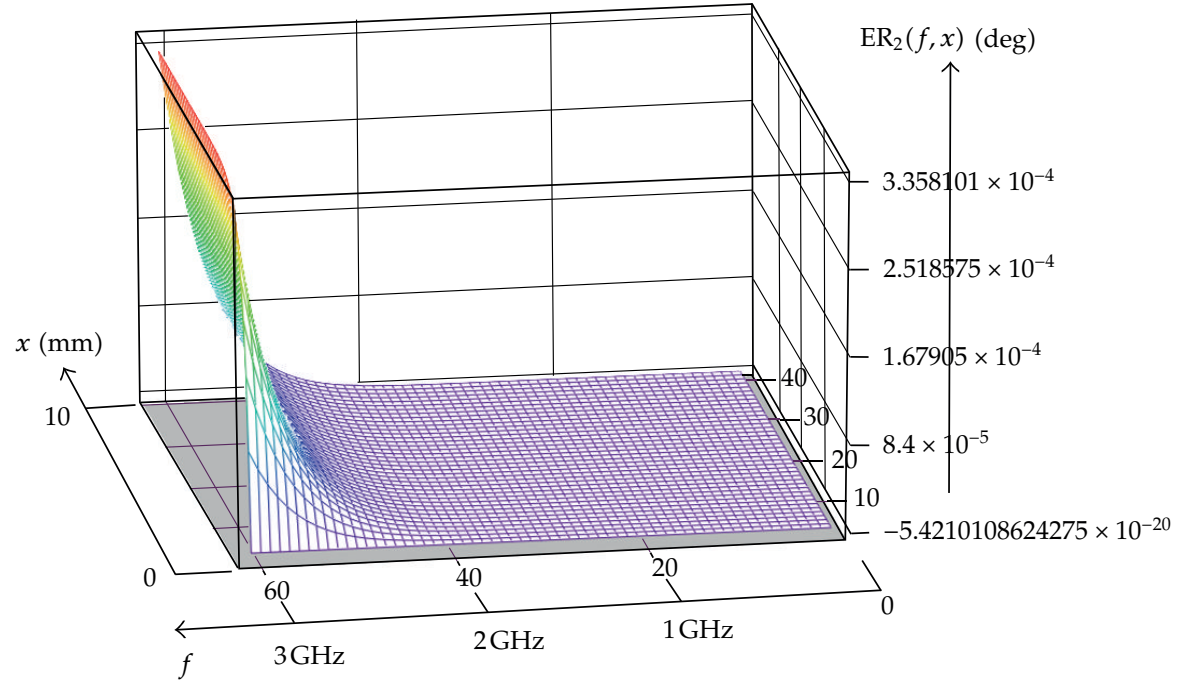

Figure 28: Voltage-transmittance phase approximation absolute error $\operatorname{ER}_{2}(f, x)$.

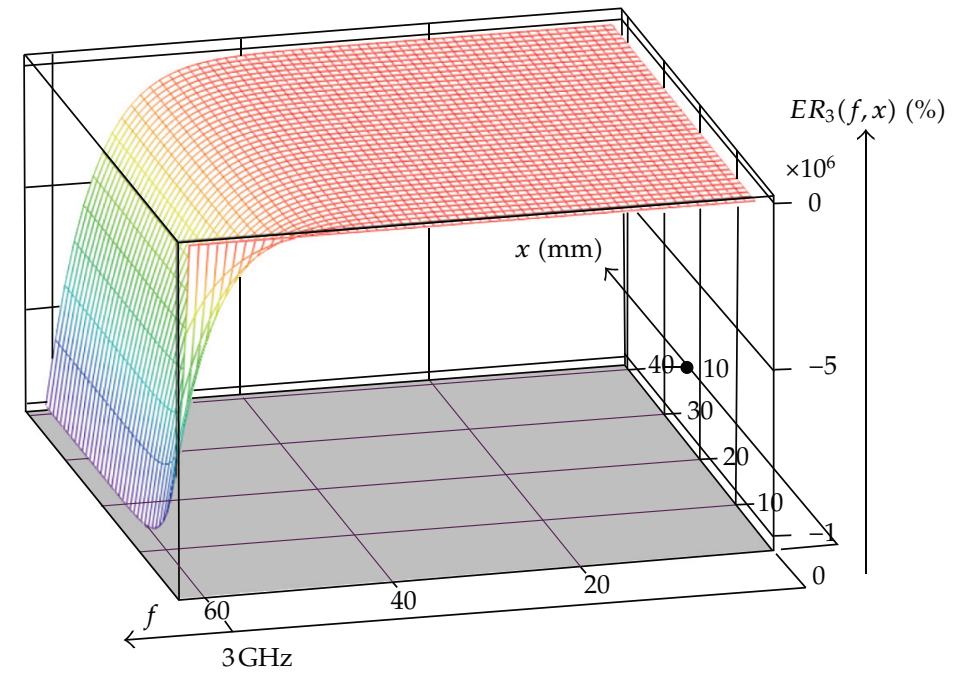

Figure 29: Current-transmittance magnitude approximation percentage error $\operatorname{ER}_{3}(f, x)$.

which, partly resembles to Maclaurin's expansion of $\cosh (\theta)$, that is, $\cosh (\theta)=1+$ $\theta^{2} / 2 !+\theta^{4} / 4 !+\ldots$

(iii) combining (i) and (ii) it follows that

$$
\tanh \left(\frac{\theta}{2}\right) \approx \tanh _{A}\left(\frac{\theta}{2}\right)=\frac{\theta \cdot\left(\theta^{2}+24\right)}{6 \cdot\left(\theta^{2}+8\right)} .
$$

The functions $\sinh (\theta) / \theta$ and $\tanh (\theta / 2) /(\theta / 2)$ play fundamental role in effort to transform short transmission line segments into equivalent lumped three-terminal RLC networks [7]. The same role is played their respective approximating functions $\sinh _{A}(\theta) / \theta$ 


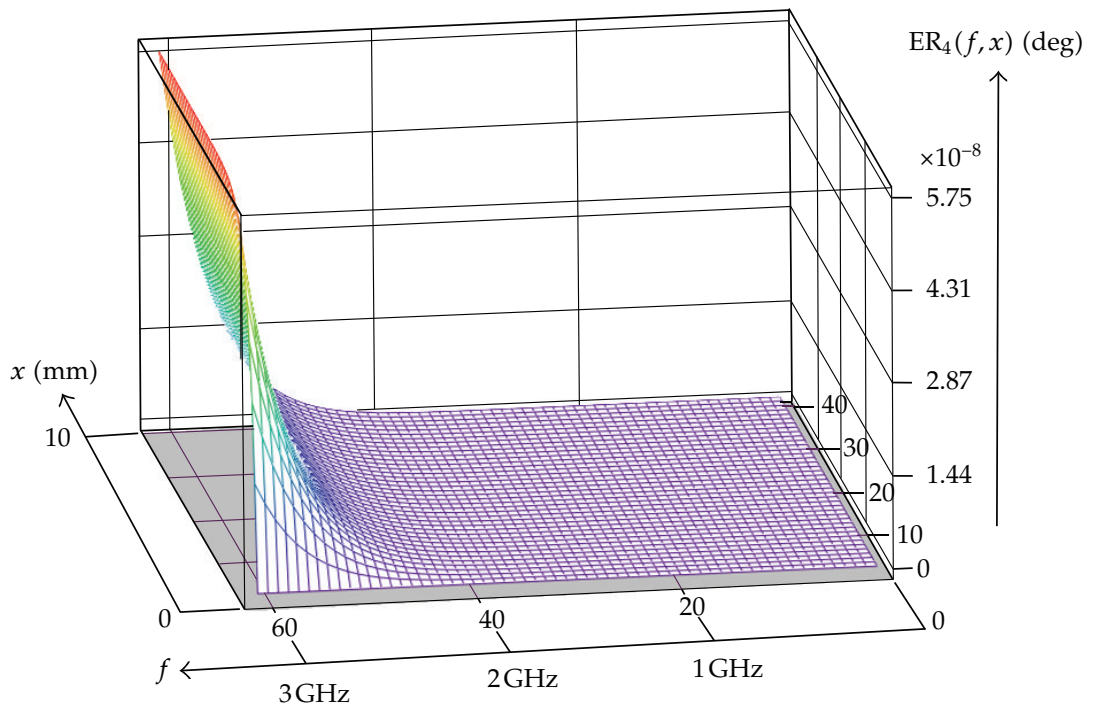

Figure 30: Current-transmittance phase approximation absolute error $\operatorname{ER}_{4}(f, x)$.

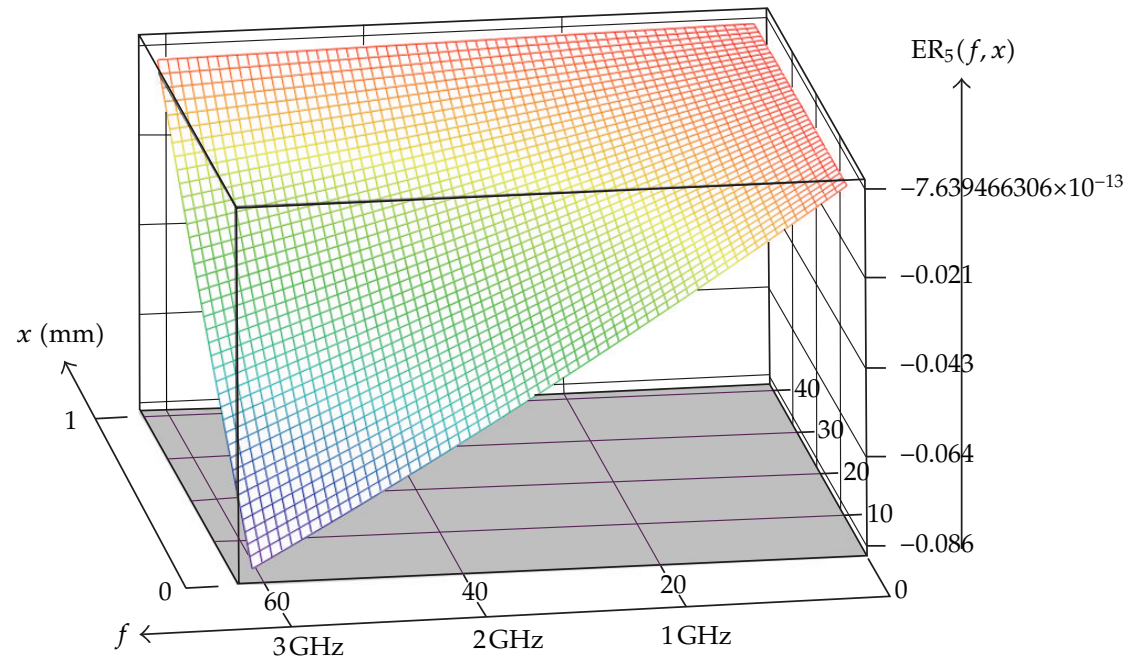

Figure 31: Magnitude approximation absolute error $\operatorname{ER}_{5}(f, x)$.

and $\tanh _{A}(\theta / 2) /(\theta / 2)$, obtained when $|\theta| \ll 1$. For two-wire line with length $\ell=1[\mathrm{~mm}]$, in Figures 31, 32, 33, and 34, the magnitude approximation absolute error $\operatorname{ER}_{5}(f, x)=$ $\left|\left(\theta+\theta^{3} / 6\right)\right|-|\sinh (\theta)|$ and three percentage magnitude approximation errors: $\operatorname{ER}_{6}(f, x)=$ $\left\{\left|\left\{\theta \cdot\left(\theta^{2}+24\right) /\left[6 \cdot\left(\theta^{2}+8\right)\right]\right\} / \tanh (\theta / 2)\right|-1\right\} \cdot 100, \operatorname{ER}_{7}(f, x)=[|\theta / \sinh (\theta)|-1] \cdot 100$ and $\mathrm{ER}_{8}(f, x)=\{|(\theta / 2) / \tanh (\theta / 2)|-1\} \cdot 100$, are depicted on $\operatorname{grid}(x) \times \operatorname{grid}(f)=40 \times 60 \mathrm{in}$ the frequency range $f \in[0,3][\mathrm{GHz}]$ and range of $x \in[0,1][\mathrm{mm}]$. Obviously, all these errors can be kept arbitrarily small in magnitude in the entire frequency range $f \in[0,3]$ $[\mathrm{GHz}]$ if sufficiently small step of uniform line segmentation is applied. The key action 


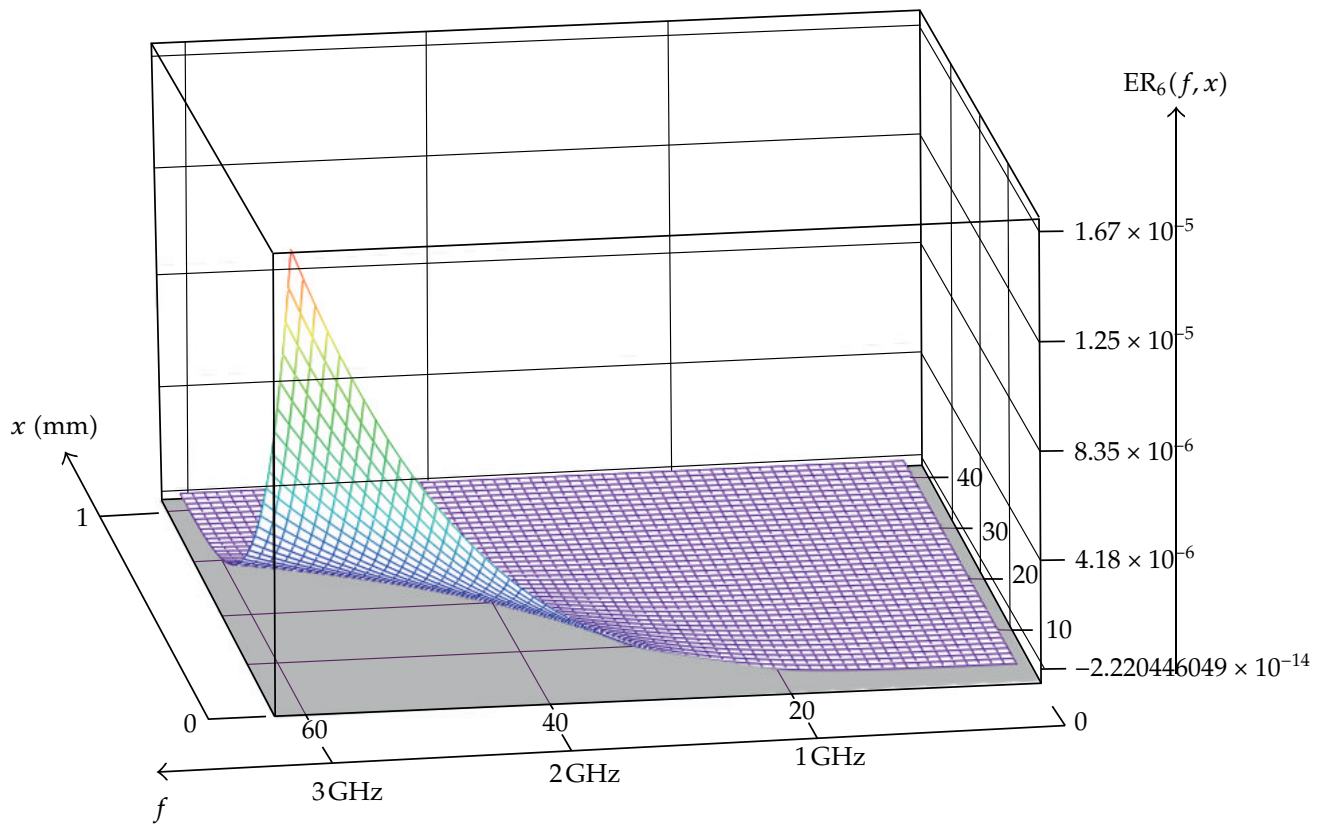

Figure 32: Percentage magnitude approximation error $\mathrm{ER}_{6}(f, x)$.

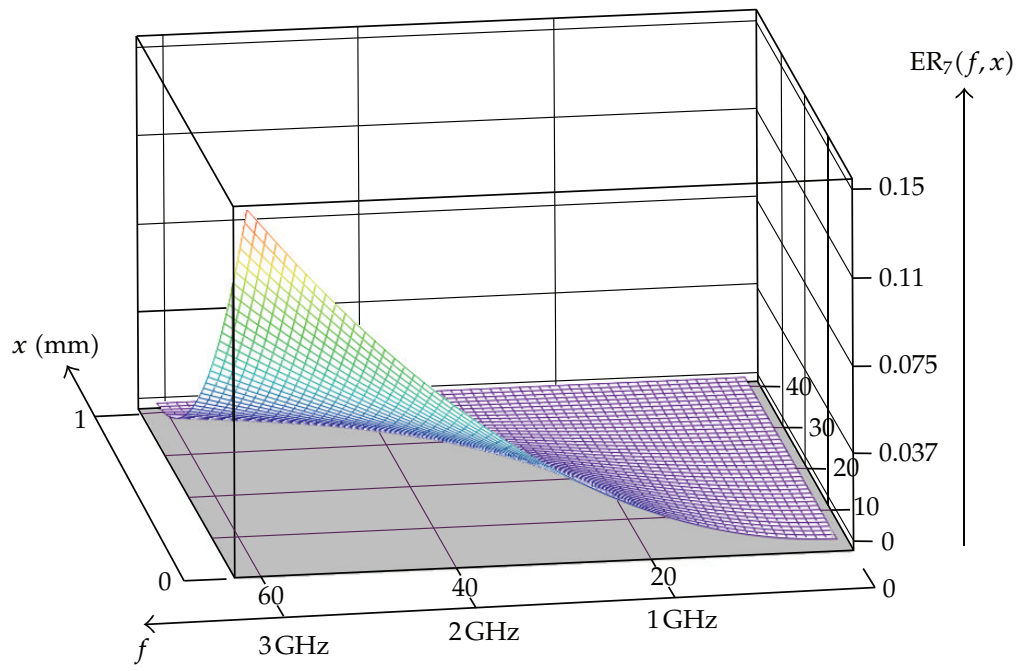

Figure 33: Percentage magnitude approximation error $\operatorname{ER}_{7}(f, x)$.

in achieving the previous goals is providing the maximum of $\ell_{0}$ to be much less than $1 / \mid \Gamma\left(j \cdot 2 \pi \cdot f_{\max }\right)\left(\Leftrightarrow\left|\theta_{\max }\right| \ll 1\right)$. For example, from the numerical data associated with Figure 17, it can be calculated that $\ell_{0}$ should be at most $1[\mathrm{~cm}]$ on the upper limit of VHF and at most $1[\mathrm{~mm}]$ on the upper limit of UHF band. Therein it has been tacitly assumed that transmission line is uniformly partitioned in segments of length $\ell_{0}$, which is at least ten times less than $1 /\left|\Gamma\left(j \cdot 2 \pi \cdot f_{\max }\right)\right|$.

The equations (A.15) and (A.16) offer an opportunity to view on a transmission line segment with length $\ell_{0}$ as on a linear two-port network (Figure 35(a)) with boundary 


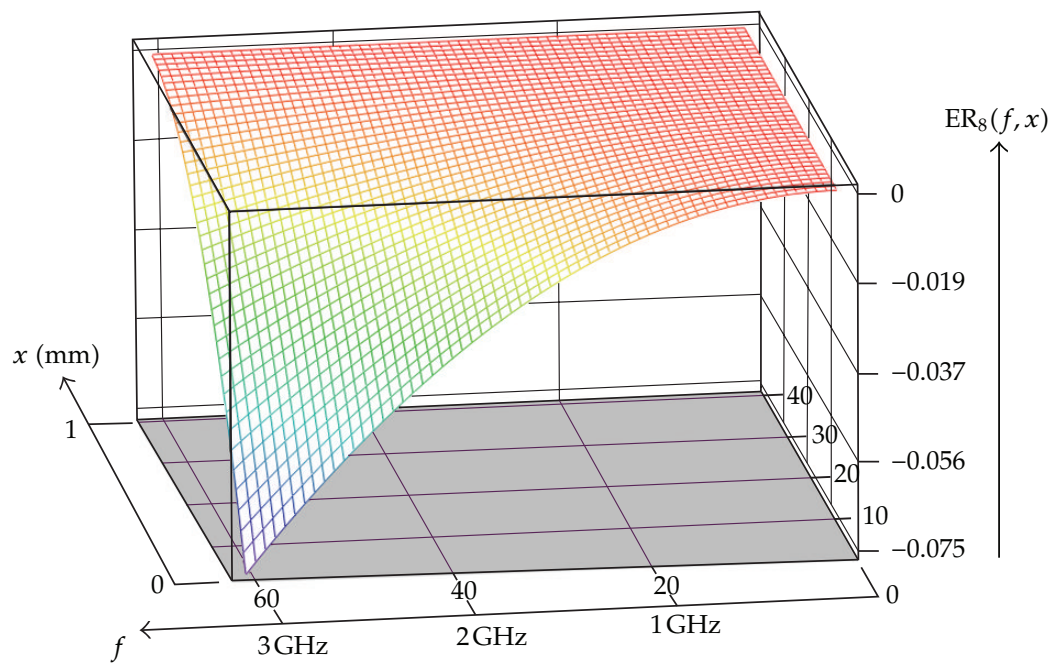

Figure 34: Percentage magnitude approximation error $\operatorname{ER}_{8}(f, x)$.

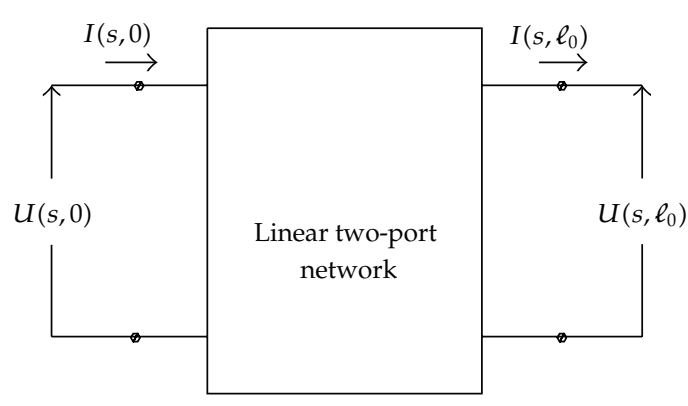

(a)

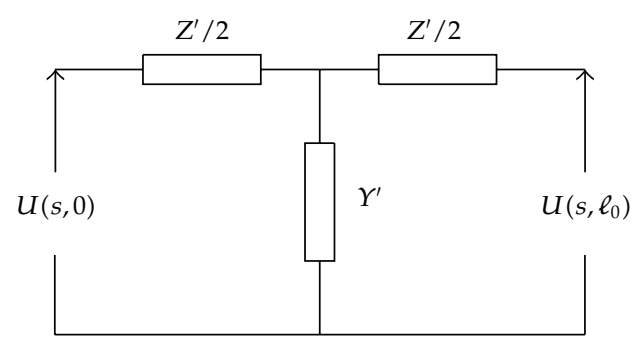

(b)

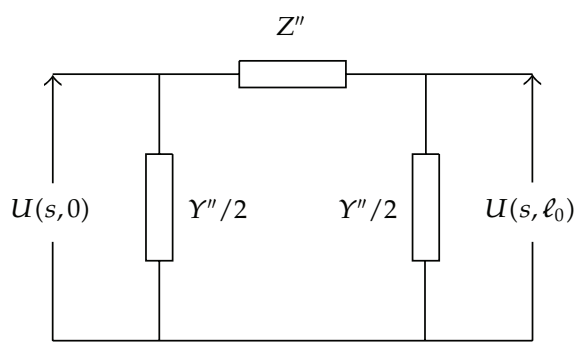

(c)

Figure 35: (a) Linear network model of a short transmission line segment and (b) its linear network structure.

conditions $U(s, 0)$ and $I(s, 0)$ at the input and $U\left(s, \ell_{0}\right)$ and $I\left(s, \ell_{0}\right)$ at the output. The chainmatrix $\mathbf{F}$ of this network reads

$$
\left[\begin{array}{c}
U(s, 0) \\
I(s, 0)
\end{array}\right]=\mathbf{F} \cdot\left[\begin{array}{c}
U\left(s, \ell_{0}\right) \\
I\left(s, \ell_{0}\right)
\end{array}\right], \quad \mathbf{F}=\left[\begin{array}{cc}
\cosh \left(\Gamma \cdot \ell_{0}\right) & Z_{0} \cdot \sinh \left(\Gamma \cdot \ell_{0}\right) \\
\frac{\sinh \left(\Gamma \cdot \ell_{0}\right)}{Z_{0}} & \cosh \left(\Gamma \cdot \ell_{0}\right)
\end{array}\right] .
$$

Denote $Z(s)=\left(R^{\prime}+L^{\prime} \cdot s\right) \cdot \ell_{0}, Y(s)=\left(G^{\prime}+C^{\prime} \cdot s\right) \cdot \ell_{0}$ and $\theta(s, 0)=\Gamma(s) \cdot \ell_{0}$. In study of short transmission lines it is found convenient to replace them, either with nominally equivalent $T$ 
networks (Figure 35(b)) or with nominally equivalent $\Pi$ networks (Figure 35(c)) [7], whose immitances are given as follows

$$
\begin{aligned}
& Z^{\prime}=Z \cdot\left\{\frac{\tanh [\theta(s, 0) / 2]}{\theta(s, 0) / 2}\right\}, \quad Y^{\prime}=Y \cdot\left\{\frac{\sinh [\theta(s, 0)]}{\theta(s, 0)}\right\}, \\
& Z^{\prime \prime}=Z \cdot\left\{\frac{\sinh [\theta(s, 0)]}{\theta(s, 0)}\right\}, \quad Y^{\prime \prime}=Y \cdot\left\{\frac{\tanh [\theta(s, 0) / 2]}{\theta(s, 0) / 2}\right\} .
\end{aligned}
$$

The aforementioned criterion for selection of $\ell_{0}$ relies on attempt to find convenient $\theta(s, 0)=$ $\Gamma(s) \cdot \ell_{0}$ that provides physical realizability of immitances $Z^{\prime}, Y^{\prime}, Z^{\prime \prime}$, and $Y^{\prime \prime}$ by lumped, transformerless $R L C$ networks. The necessary and sufficient condition for existence and realizability of these immitances is that they must be rational, positive real functions in complex frequency $s$ [10]. Observe that the immitances $Z(s)$ and $Y(s)$ are realizable by trivial two-element-kind RLC networks. Nevertheless, we will show now that, in general, the imitances $Z^{\prime}, Y^{\prime}, Z^{\prime \prime}$, and $Y^{\prime \prime}$ are not realizable by lumped $R L C$ networks, except in the limiting case when $\ell_{0}$ is as small, so that the complex approximations hold: $\sinh (\theta) / \theta \approx 1$ and $\tanh (\theta / 2) /(\theta / 2) \approx 1$ (observe that if $\theta \rightarrow 0$, then $Z^{\prime}$ and $Z^{\prime \prime} \rightarrow Z$ and $Y^{\prime}$ and $Y^{\prime \prime} \rightarrow Y$ ). To see that, recall that for $\Gamma(j \cdot \omega)=\sqrt{\left(R^{\prime}+j \cdot \omega \cdot L^{\prime}\right) \cdot\left(G^{\prime}+j \cdot \omega \cdot C^{\prime}\right)}=|\Gamma(j \cdot \omega)| \cdot \exp \{j \cdot \arg [\Gamma(j \cdot$ $\omega)]\}(\omega=2 \pi \cdot f)$, in frequency range $f \in[0.01,3][\mathrm{GHz}]$ it holds

(a) $\omega \cdot L^{\prime} / R^{\prime} \wedge \omega /\left(R^{\prime} / L^{\prime}+G^{\prime} / C^{\prime}\right) \in[32.652,609.312] \gg 1, \omega \cdot C^{\prime} / G^{\prime} \in\left[1.257 \cdot 10^{12}, 3.771\right.$. $\left.10^{14}\right] \gg 1$,

(b) $|\Gamma(j \cdot \omega)| \approx \omega \cdot\left(L^{\prime} \cdot C^{\prime}\right)^{1 / 2} \in[0.319,94.598], \arg [\Gamma(j \cdot \omega)] \in[89.122,89.952]$ [deg],

(c) $\arg [\Gamma(j \cdot \omega)]=\pi / 2-\varepsilon(\omega)$, where,

$$
\begin{aligned}
\varepsilon(\omega) & =\frac{\pi}{2}-\arg [\Gamma(j \cdot \omega)]=\frac{\pi}{2}-\frac{1}{2} \cdot a \tan \left(\frac{\left(\omega \cdot L^{\prime}\right) / R^{\prime}+\left(\omega \cdot C^{\prime}\right) / G^{\prime}}{1-\left(L^{\prime} \cdot C^{\prime}\right) /\left(R^{\prime} \cdot G^{\prime}\right) \cdot \omega^{2}}\right) \\
& \approx \frac{1}{2} \cdot a \tan \left(\frac{R^{\prime} / L^{\prime}+G^{\prime} / C^{\prime}}{\omega}\right) \approx \frac{R^{\prime} / L^{\prime}+G^{\prime} / C^{\prime}}{2 \cdot \omega} .
\end{aligned}
$$

(d) $\Gamma(j \cdot \omega)=|\Gamma(j \cdot \omega)| \cdot\{\sin [\varepsilon(\omega)]+j \cdot \cos [\varepsilon(\omega)]\}, \varepsilon(\omega) \in(0, \pi / 200)$ and $\theta(j \cdot \omega, x)=$ $\Gamma(j \cdot \omega) \cdot\left(\ell_{0}-x\right)=|\theta(j \cdot \omega, x)| \cdot\{\sin [\varepsilon(\omega)]+j \cdot \cos [\varepsilon(\omega)]\}$. Observe that $\theta(j \cdot \omega, x)$ is produced as almost pure imaginary number.

(e) Since $x \in\left[0, \ell_{0}\right]$ and $|\theta(j \cdot \omega, x)| \approx \omega \cdot\left(L^{\prime} \cdot C^{\prime}\right)^{1 / 2} \cdot\left(\ell_{0}-x\right)=A(\omega, x)$, then by selecting $\ell_{0}$ sufficiently small, $A(\omega, x)$ can always be produced arbitrarily small. following:

Bearing in mind the properties $(\mathrm{a}) \div(\mathrm{e})$, we obtain for $\sinh [\theta(j \cdot \omega, x)] / \theta(j \cdot \omega, x)$ the

$$
\begin{aligned}
\frac{\sinh \{A(\omega, x) \cdot \sin [\varepsilon(\omega)]\} \cdot \cos \{A(\omega, x) \cdot \cos [\varepsilon(\omega)]\}}{A(\omega, x) \cdot\{\sin [\varepsilon(\omega)]+j \cdot \cos [\varepsilon(\omega)]\}} \\
+\frac{j \cdot \cosh \{A(\omega, x) \cdot \sin [\varepsilon(\omega)]\} \cdot \sin \{A(\omega, x) \cdot \cos [\varepsilon(\omega)]\}}{A(\omega, x) \cdot\{\sin [\varepsilon(\omega)]+j \cdot \cos [\varepsilon(\omega)]\}},
\end{aligned}
$$


that can be rewritten as $\sinh [\theta(j \cdot \omega, x)] / \theta(j \cdot \omega, x)=R(\omega, x)+j \cdot Q(\omega, x) \cdot R(\omega, x)$ and $Q(\omega, x)$ are even and odd functions in $\omega$, respectively, which are represented with the following expanded forms:

$$
\begin{aligned}
R(\omega, x)= & \sin ^{2}[\varepsilon(\omega)] \cdot\left(\sum_{k=0}^{\infty} \frac{A^{2 k}(\omega, x) \cdot \sin ^{2 k}[\varepsilon(\omega)]}{(2 k+1) !}\right) \cdot\left(\sum_{m=0}^{\infty}(-1)^{m} \frac{A^{2 m}(\omega, x) \cdot \cos ^{2 m}[\varepsilon(\omega)]}{(2 m) !}\right) \\
+ & \cos ^{2}[\varepsilon(\omega)] \cdot\left(\sum_{n=0}^{\infty} \frac{A^{2 n}(\omega, x) \cdot \sin ^{2 n}[\varepsilon(\omega)]}{(2 n) !}\right) \cdot\left(\sum_{p=0}^{\infty}(-1)^{p} \frac{A^{2 p}(\omega, x) \cdot \cos ^{2 p}[\varepsilon(\omega)]}{(2 p+1) !}\right), \\
Q(\omega, x)=\sin [\varepsilon(\omega)] \cdot \cos [\varepsilon(\omega)] & \cdot\left\{\left(\sum_{k=0}^{\infty} \frac{A^{2 k}(\omega, x) \cdot \sin ^{2 k}[\varepsilon(\omega)]}{(2 k) !}\right) \cdot\left(\sum_{m=0}^{\infty}(-1)^{m} \frac{A^{2 m}(\omega, x) \cdot \cos ^{2 m}[\varepsilon(\omega)]}{(2 m+1) !}\right)\right. \\
& \left.-\left(\sum_{n=0}^{\infty} \frac{A^{2 n}(\omega, x) \cdot \sin ^{2 n}[\varepsilon(\omega)]}{(2 n+1) !}\right) \cdot\left(\sum_{p=0}^{\infty}(-1)^{p} \frac{A^{2 p}(\omega, x) \cdot \cos ^{2 p}[\varepsilon(\omega)]}{(2 p) !}\right)\right\} .
\end{aligned}
$$

We must always bear in mind that $\varepsilon(\omega)$ is small $[(c)$ and $(2.30)]$ and that $A(\omega, x)=\omega$. $\left(L^{\prime} \cdot C^{\prime}\right)^{1 / 2} \cdot\left(\ell_{0}-x\right)$ can be made arbitrarily small by selecting $\ell_{0}$ such that $\left.A(\omega, \ell)\right|_{\max }=$ $\left.\left[2 \pi \cdot f \cdot\left(L^{\prime} \cdot C^{\prime}\right)^{1 / 2}\right]\right|_{\max } \cdot \ell_{0} \ll 1$ or equivalently $\ell_{0} \ll 1 /\left|\Gamma\left(j \cdot 2 \pi \cdot f_{\max }\right)\right|$. Then, retaining only the first two terms in each of the convergent infinite sums in (2.32), the approximations of $R(\omega, x)$ and $Q(\omega, x)$ are obtained which hold for all $x \in\left[0, \ell_{0}\right]$ and for all $\omega$ corresponding to $f$ from the specified frequency range:

$$
\begin{aligned}
R(\omega, x) & \approx 1-\frac{A^{2}(\omega, x)}{6} \cdot \cos [2 \cdot \varepsilon(\omega)]-\frac{A^{4}(\omega, x)}{48} \cdot \sin ^{2}[2 \cdot \varepsilon(\omega)] \approx 1-\frac{\omega^{2} \cdot L^{\prime} \cdot C^{\prime} \cdot\left(\ell_{0}-x\right)^{2}}{6} \\
Q(\omega, x) & \approx \frac{A^{2}(\omega, x)}{6} \cdot \sin [2 \cdot \varepsilon(\omega)] \approx \frac{\omega^{2} \cdot L^{\prime} \cdot C^{\prime} \cdot\left(\ell_{0}-x\right)^{2}}{6} \cdot \sin [2 \cdot \varepsilon(\omega)] \\
& \approx \frac{\omega^{2} \cdot L^{\prime} \cdot C^{\prime} \cdot\left(\ell_{0}-x\right)^{2}}{3} \cdot \varepsilon(\omega)=\frac{\omega}{6} \cdot L^{\prime} \cdot C^{\prime} \cdot\left(\ell_{0}-x\right)^{2} \cdot\left(\frac{R^{\prime}}{L^{\prime}}+\frac{G^{\prime}}{C^{\prime}}\right) \\
& =\frac{\omega}{6} \cdot\left(\ell_{0}-x\right)^{2} \cdot\left(R^{\prime} \cdot C^{\prime}+G^{\prime} \cdot L^{\prime}\right) \ll 1 .
\end{aligned}
$$

For $x=0$, from (2.31) and (2.33) it finally follows:

$$
\begin{aligned}
& \frac{\sinh [\theta(j \cdot \omega, 0)]}{\theta(j \cdot \omega, 0)}=R(\omega, 0)+j \cdot Q(\omega, 0) \approx 1+j \cdot \frac{\omega}{6} \cdot\left(R^{\prime} \cdot C^{\prime}+G^{\prime} \cdot L^{\prime}\right) \cdot \ell_{0}^{2}-\frac{\omega^{2} \cdot L^{\prime} \cdot C^{\prime} \cdot \ell_{0}^{2}}{6}, \\
& \frac{\sinh [\theta(j \cdot \omega, 0)]}{\theta(j \cdot \omega, 0)} \underset{\text { By analytic continuation }}{\stackrel{j \cdot \omega \rightarrow s\left(-\omega^{2} \rightarrow s^{2}\right)}{\longrightarrow}} \frac{\sinh [\theta(s, 0)]}{\theta(s, 0)} \approx 1+\frac{s}{6} \cdot\left(R^{\prime} \cdot C^{\prime}+G^{\prime} \cdot L^{\prime}\right) \cdot \ell_{0}^{2}+\frac{s^{2} \cdot L^{\prime} \cdot C^{\prime} \cdot \ell_{0}^{2}}{6} .
\end{aligned}
$$


Now, by using (2.29) and (2.35) we may generate the immitances $Y^{\prime}$ and $Z^{\prime \prime}$ (Figures $35(b)$ and $35(c))$,

$$
\begin{aligned}
& Y^{\prime}(s)=Y(s) \cdot \frac{\sinh [\theta(s, 0)]}{\theta(s, 0)}=\left(G^{\prime}+C^{\prime} \cdot s\right) \cdot \ell_{0} \cdot\left[1+\frac{s}{6} \cdot\left(R^{\prime} \cdot C^{\prime}+G^{\prime} \cdot L^{\prime}\right) \cdot \ell_{0}^{2}+\frac{s^{2} \cdot L^{\prime} \cdot C^{\prime} \cdot \ell_{0}^{2}}{6}\right], \\
& Z^{\prime \prime}(s)=Z(s) \cdot \frac{\sinh [\theta(s, 0)]}{\theta(s, 0)}=\left(R^{\prime}+L^{\prime} \cdot s\right) \cdot \ell_{0} \cdot\left[1+\frac{s}{6} \cdot\left(R^{\prime} \cdot C^{\prime}+G^{\prime} \cdot L^{\prime}\right) \cdot \ell_{0}^{2}+\frac{s^{2} \cdot L^{\prime} \cdot C^{\prime} \cdot \ell_{0}^{2}}{6}\right],
\end{aligned}
$$

which are not realizable by lumped $R L C$ networks, since they are not the positive real functions in complex frequency $s[10]$, except when $\ell_{0} \rightarrow 0$. Putting it in other words we may say that if in the whole operating frequency range practically hold the three conditions: $\omega \cdot L^{\prime} / R^{\prime} \gg 1$, $\omega \cdot C^{\prime} / G^{\prime} \gg 1$, and $\ell_{0} \cdot \omega \cdot \sqrt{L^{\prime} \cdot C^{\prime}} \ll 1$, the immitances $Y^{\prime}(s)$ and $Z^{\prime \prime}(s)$ (Figures 35(b) and 35 (c)) are produced in the following simple form and are realizable by two-element-kind $R L C$ networks [see (2.36) and (2.37)]:

$$
Y^{\prime}(s)=\left(G^{\prime}+C^{\prime} \cdot s\right) \cdot \ell_{0}, \quad Z^{\prime \prime}(s)=\left(R^{\prime}+L^{\prime} \cdot s\right) \cdot \ell_{0} .
$$

Relations (2.38) may be considered as a consequence of approximation $\sinh [\theta(s, 0)] \approx$ $[\theta(s, 0)]$ applied to $(2.36)$ and $(2.37)$ when $\theta(s, 0) \rightarrow 0$. Similarly, under the conditions: $\omega \cdot L^{\prime} / R^{\prime} \gg 1, \omega \cdot C^{\prime} / G^{\prime} \gg 1$ and $\ell_{0} \cdot \omega \cdot \sqrt{L^{\prime} \cdot C^{\prime}} \ll 1$, the complex approximation $\tanh [\theta(s, 0) / 2] \approx[\theta(s, 0) / 2]$ applied to $(2.29)$ when $\theta(s, 0) \rightarrow 0$ gives the other two immitances from (2.29), which are necessary to accomplish forming of linear networks in Figures 35(b) and 35(c), which are nominally equivalent to the network in Figure 35(a).

$$
Z^{\prime}(s)=\left(R^{\prime}+L^{\prime} \cdot s\right) \cdot \ell_{0}, \quad Y^{\prime \prime}(s)=\left(G^{\prime}+C^{\prime} \cdot s\right) \cdot \ell_{0} .
$$

\section{Approximation of Two-Wire Line by Uniform RLCG Ladder and the Simulation Results}

Let us consider a short two-wire line with length $\ell=30[\mathrm{~mm}]$ (or a longone partitioned in sections of length $\ell$ ). Assume that the bandwith of the signal being transmitted is $B<$ $770[\mathrm{kHz}]$ and that its central frequency is $f_{0}=1[\mathrm{GHz}]$. Then, make the graph of function $\left.l(f)=\phi_{3}(f)=1 / 2 \pi \cdot f \cdot\left[L^{\prime}(f) \cdot C^{\prime}\right)^{1 / 2}\right] \approx 1 /|\Gamma(j \cdot 2 \pi \cdot f)|$ (Figure 36) and from its associated numerical data find that $l\left(10^{9}\right) \approx 31.7[\mathrm{~mm}]$. Let the maximum length $\ell_{0}$ of line segments be selected to satisfy the condition $\ell_{0} \leq l\left(10^{9}\right) / 10 \approx 3.17[\mathrm{~mm}]$. Finally, assume $\ell_{0}=3[\mathrm{~mm}]$ and calculate the number of cells $N=\ell / \ell_{0}=10$ in uniform $R L C G$ ladder purporting to represent the transmission line with length $\ell$ in frequency range $f \in\left[f_{0}-B / 2, f_{0}+B / 2\right]$.

Assume $\delta x=\ell_{0}$ and calculate the parameters of uniform lumped RLCG network representing the line segments of length $\ell_{0}$ (see Figure 2). If the overall short-line parameters are $R=R^{\prime} \cdot \ell$-resistance, $L=L^{\prime} \cdot \ell$-inductance, $C=C^{\prime} \cdot \ell$-capacitance, and $G=G^{\Gamma(j \cdot 2 \pi \cdot f)} \cdot \ell$ conductance, then the lumped $R L C G$ network parameters: $R^{\prime} \cdot \delta x / 2=R / 20, L^{\prime} \cdot \delta x / 2=L / 20$, $C^{\prime} \cdot \delta x=C / 10$, and $G^{\prime} \cdot \delta x=G / 10$, calculated at frequency $f_{0}=1[\mathrm{GHz}]$ according to (2.1), (2.2), ((2.8)-(2.11)), are given in Table 1. The RLCG network representing the short 
Table 1: Two-wire copper-polyethylene transmission line (central frequency $f_{0}=1[\mathrm{GHz}]$ ).

\begin{tabular}{lcccc}
\hline Electrical parameters of $R L C G$ ladder sections & $R / 20[\mathrm{~m} \Omega]$ & $L / 20[\mathrm{nH}]$ & $C / 10[\mathrm{fF}]$ & $G / 10[\mathrm{aS}]$ \\
\hline & 39.772 & 2.219 & 51.123 & 2.554 \\
\hline
\end{tabular}

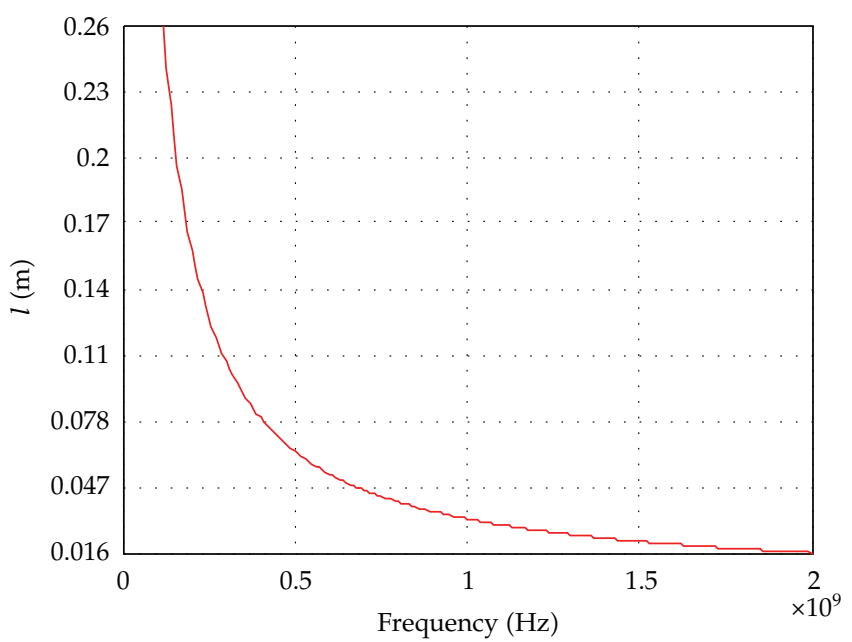

Figure 36: The function $l(f)=\phi_{3}(f)$ of the considered two-wire line (determination of $\ell_{0}$ ).

line with length $\ell$ is depicted in Figure 37, whereon the conductance elements G/10 (present in Figure 2) are omitted only for the simplicity of drawing but are included in the PSPICE simulation network. Also, observe that at frequency $f_{0}=1[\mathrm{GHz}]$ the quantity $2 \pi \cdot f_{0} \cdot C^{\prime} / G^{\prime}$ takes on extremely high value. Let $u_{i}$ be the voltage of the point $N_{i}(i=\overline{1,41})$, with respect to the common node 0 (Figure 37).

Now we will present the results obtained by PSPICE simulation of the open-circuited network in Figure 37 and compare them to the results obtained by exact analysis of considered open-circuited line. The amplitude of excitation voltage $e$ in simulation was 1 [V], its frequency was $f_{0}=1[\mathrm{GHz}]$ and the initial phase $0[\mathrm{deg}]$. The steady-state, odd numbered point voltages, and their phase angles obtained through PSPICE analysis of open-circuited network in Figure 37, are summarized in Table 2. Also, in this table the exactly obtained voltages at points on the line with distance $x_{m}=(m-1) \cdot \ell_{0} / 2(m=\overline{1,21})$ from the line sending end are presented. To these points correspond the points nodes $N_{2 m-1}(m=\overline{1,21})$ in the simulation RLCG ladder on Figure 37.

When analysis of long lines is considered in the time domain it is useful to resort to forming of multilevel hierarchical blocks in PSPICE. To see that, suppose that we are to consider transmission of a signal with frequency $f_{0}=1[\mathrm{GHz}]$, amplitude $E_{m}=10[\mathrm{~V}]$, and zero initial phase in two-wire line with length $\ell=4.5[\mathrm{~m}]$ and parameters as in Table 1. Let us designate the ladder on Figure 37, which represents a two-wire line with length 3 [cm], as level 1 hierarchical block $\mathrm{HB}_{1}$ on Figure 38(a). By cascading, say, 30 level 1 blocks: $\mathrm{HB}_{1} / 1, \mathrm{HB}_{1} / 2, \ldots$, and $\mathrm{HB}_{1} / 30$, we produce a level 2 hierarchical block $\mathrm{HB}_{2}$ on Figure $38(\mathrm{~b})$, which represents a two-wire line with length 90 [cm]. By cascading 5 level 2 blocks: $\mathrm{HB}_{2} / 1$, $\mathrm{HB}_{2} / 2, \ldots$, and $\mathrm{HB}_{2} / 5$, we make a level 3 hierarchical block $\mathrm{HB}_{3}$ on Figure 38(c), which represents a two-wire line with length $450[\mathrm{~cm}]$, and so on. Hierarchical blocks of arbitrary length may be considered as independent entities or sophisticated parts in PSPICE. 


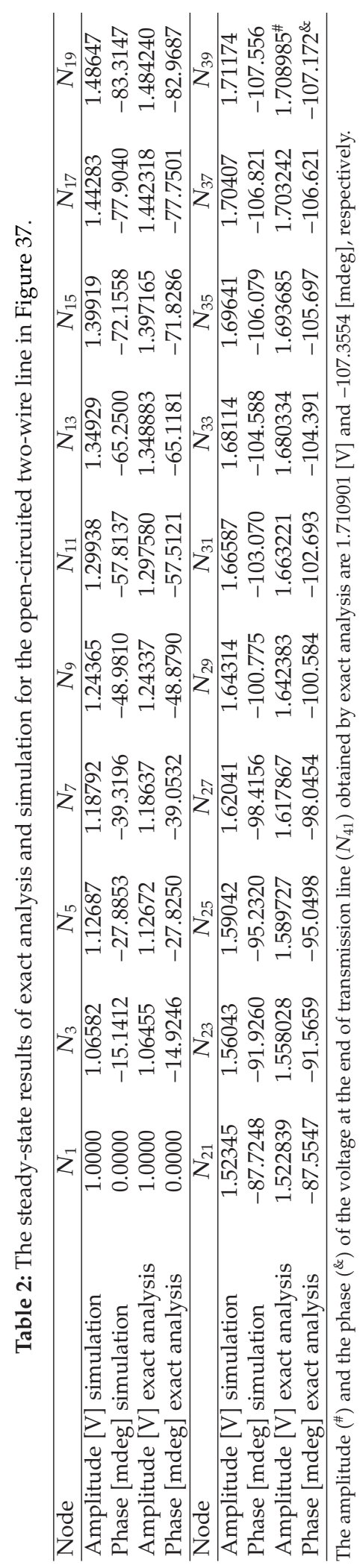




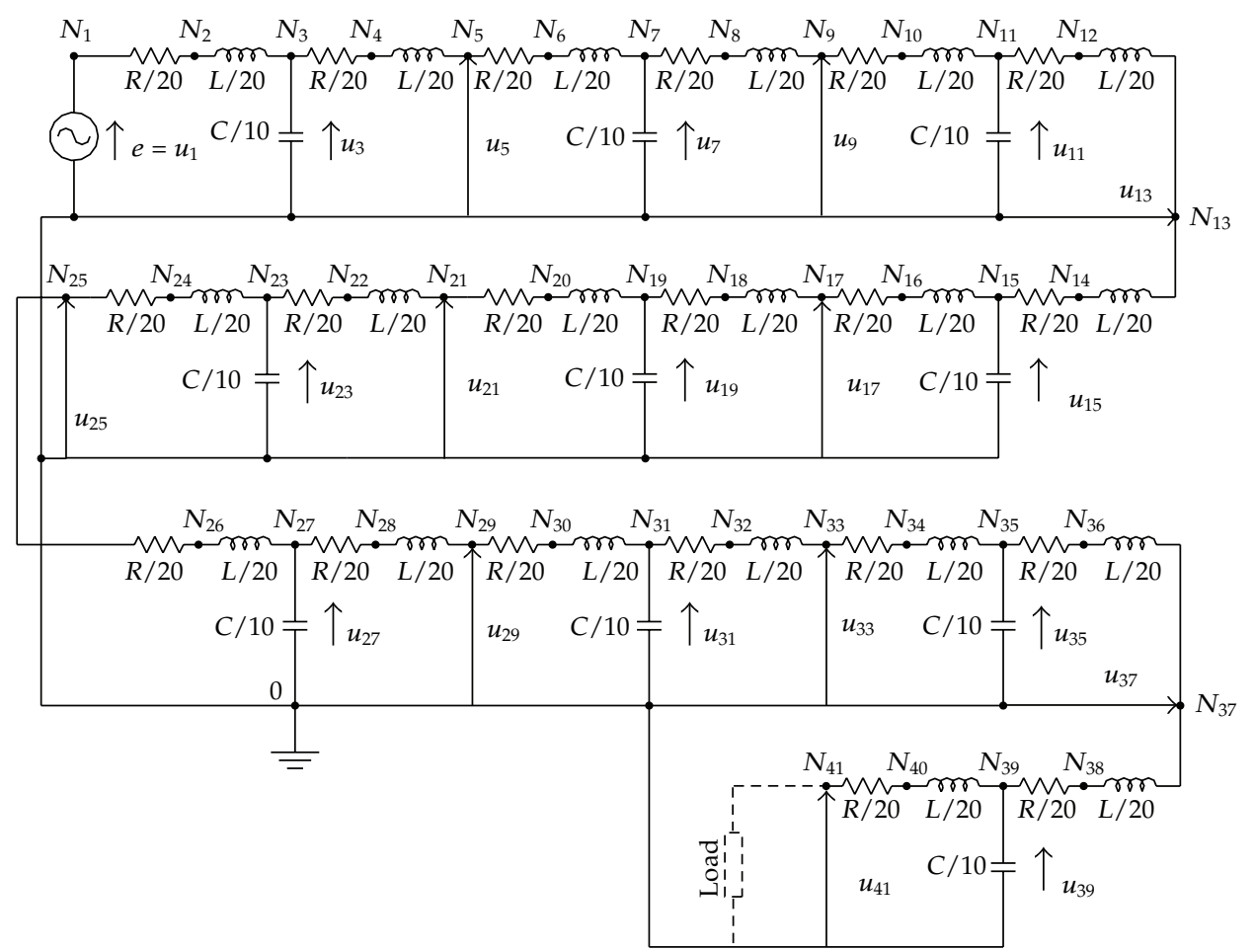

Figure 37: Electrical network model of two-wire line with length $\ell=3[\mathrm{~cm}]$ in the frequency range $f \in$ $\left[f_{0}-B / 2, f_{0}+B / 2\right]$.

From the data associated with functions depicted in Figures 12 and 13 we can easily: (i) obtain $Z_{0}\left(j \cdot 2 \pi \cdot f_{0}\right)=294.67280-j \cdot 0.42017[\Omega]$ and (ii) notice that variations of $\left.\left|Z_{0}(j \cdot 2 \pi \cdot f)\right|\right]$ and $\operatorname{Arg}\left[Z_{0}(j \cdot 2 \pi \cdot f)\right]$ are very small in the frequency range $\left(f_{0}-B / 2, f_{0}+B / 2\right)$.

Now, suppose that the block $\mathrm{HB}_{2} / 5$ in Figure 38(c) is terminated with impedance, $Z_{L}\left(j \cdot 2 \pi \cdot f_{0}\right)=R_{L}+1 /\left(j \cdot 2 \pi \cdot f_{0} \cdot C_{L}\right)=Z_{0}\left(j \cdot 2 \pi \cdot f_{0}\right)$ and find $R_{L}=294.6728[\Omega]$ and $C_{L}=378.7781[\mathrm{pF}]$. From (A.17) it follows that $M\left(j \cdot 2 \pi \cdot f_{0}, x\right)=N\left(j \cdot 2 \pi \cdot f_{0}, x\right)=\exp [-x$. $\left.\Gamma\left(j \cdot 2 \pi \cdot f_{0}\right)\right]=\exp \left[-x \cdot a\left(f_{0}\right)\right] \cdot\left\{\cos \left[x \cdot b\left(f_{0}\right)\right]-j \cdot \sin \left[x \cdot b\left(f_{0}\right)\right]\right\}$, and then at any place $x$ on the line we obtain the exact values of signal amplitude $E_{m} \cdot \exp \left[-x \cdot a\left(f_{0}\right)\right][\mathrm{V}]$, phase delay $\varphi(x)=x \cdot b\left(f_{0}\right)$ [rad] and time delay $\tau(x)=x \cdot b\left(f_{0}\right) /\left(2 \pi \cdot f_{0}\right)$ [s]. In Figure 20 we see that function $b(f)$ is practically linear in $f$, so that $\tau(x)$ should be linear in $x$, too. To verify the ladder model of two-wire line with length $\ell=4.5[\mathrm{~m}]$ (Figure 38(c)), we will compare the exact values of $E_{m} \cdot \exp \left[-x \cdot a\left(f_{0}\right)\right], \varphi(x)$ and $\tau(x)$ at the points on line $x=x_{k}=k \cdot \ell_{0} / 2(k=$ $\overline{1,3000})\left(\ell_{0}=3[\mathrm{~mm}]\right)$, with values obtained by PSPICE simulation. Bearing in mind the topological uniformity of the considered ladder, it is felt that for estimation of the proposed model it will suffice to check the ladder response only at the selected set of points whose voltages are $u_{i}(i=\overline{1,5})$ with respect to the common node 0 (i.e., ground) (Figure 38(c)). The distances of these five points from the line sending end are $x_{600 \cdot i}=300 \cdot i \cdot \ell_{0}=90 \cdot i[\mathrm{~cm}]$ $(i=\overline{1,5})$, respectively. For these voltages in Table 3 are given the steady-state results of both the simulation and the exact analysis, where the following notation has been used (Figures 19,20 , and 38(c)):

$$
\begin{gathered}
E_{m}=10[\mathrm{~V}], \quad f_{0}=1[\mathrm{GHz}], \quad a\left(f_{0}\right)=0.044990161\left[\frac{1}{\mathrm{~m}}\right], \quad b\left(f_{0}\right)=31.551675282\left[\frac{1}{\mathrm{~m}}\right], \\
e=E_{m} \cdot \sin \left(2 \pi \cdot f_{0} \cdot t\right), \quad u_{i}=U_{i m} \cdot \sin \left(2 \pi \cdot f_{0} \cdot t-\varphi_{i}\right)=U_{i m} \cdot \sin \left[2 \pi \cdot f_{0} \cdot\left(t-\tau_{i}\right)\right],
\end{gathered}
$$




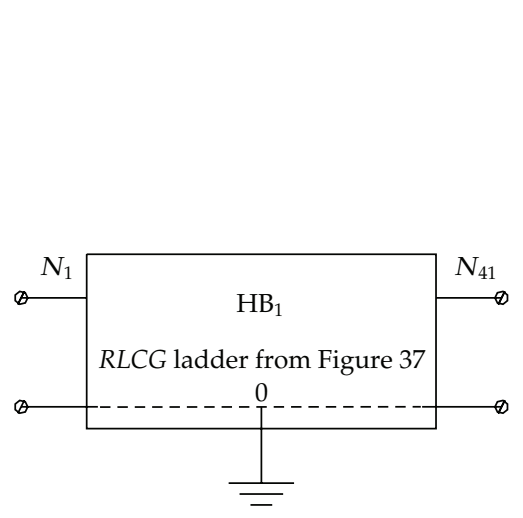

(a)

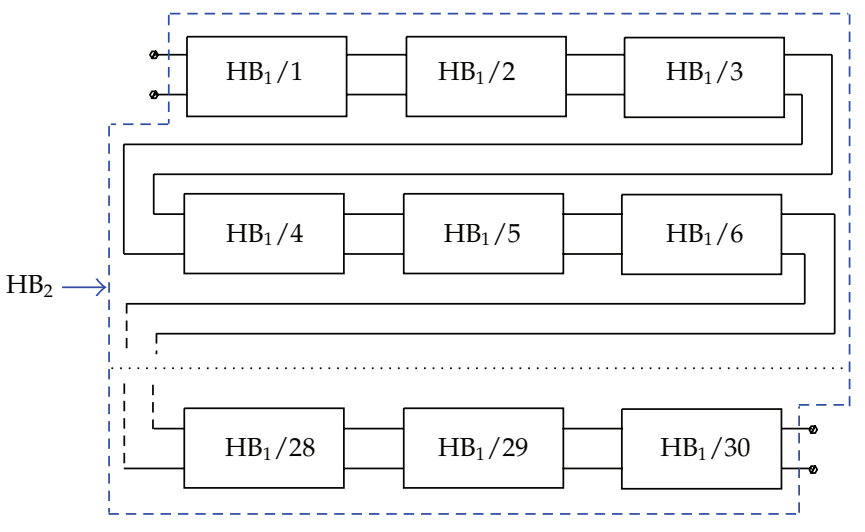

(b)

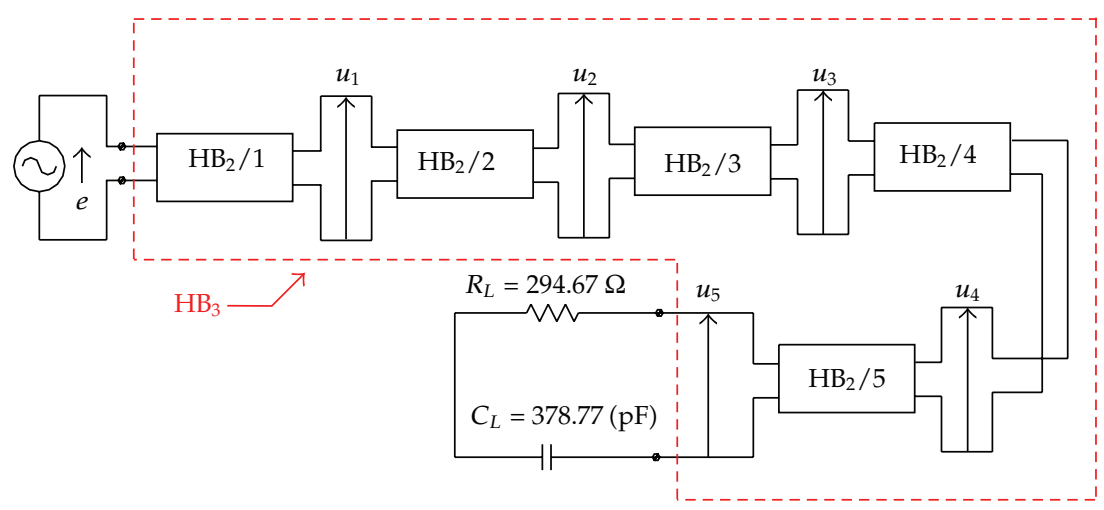

(c)

Figure 38: The structure of the three types of hierarchical blocks $\mathrm{HB}_{1}, \mathrm{HB}_{2}$, and $\mathrm{HB}_{3}$ used in PSPICE simulation.

$$
\begin{gathered}
U_{i m}=E_{m} \cdot \exp \left[-x_{600 \cdot i} \cdot a\left(f_{0}\right)\right]=10 \cdot(0.960317668)^{i}[\mathrm{~V}] \\
\varphi_{i}=\varphi\left(x_{600 \cdot i}\right)=300 \cdot i \cdot \ell_{0} \cdot b\left(f_{0}\right)=28.39650775 \cdot i[\mathrm{rad}], \\
\tau_{i}=\tau\left(x_{600 \cdot i}\right)=\frac{\varphi_{i}}{2 \pi \cdot f_{0}}=4.519444575 \cdot i[\mathrm{~ns}], \quad(i=\overline{1,5}) .
\end{gathered}
$$

From Table 3 it is evident the good agreement between the simulation results and those obtained by exact analysis. The selection of less segmentation step $\ell_{0}$ will improve this agreement at the expense of rising the complexity of RLCG network, as a consequence of proliferation in number of network elements.

In Figure 39 the results of transient PSPICE analysis of RLCG ladder with zero initial conditions (Figure 38(c)) representing the approximate network model of two-wire line with $\ell=4.5[\mathrm{~m}]$ are depicted.

Now we can summarize our obtained results

(i) A transmission line is physically dispersive system with respect to frequency, having the infinite number of poles and zeros and complex transient dynamics, which cannot be represented perfectly with common-ground ladder with possibly great, but finite number of RLCG elements. 


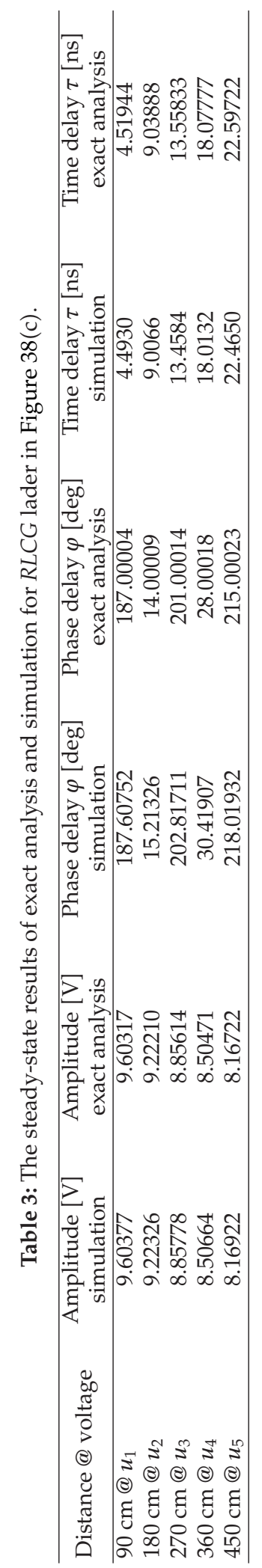


Transient analysis

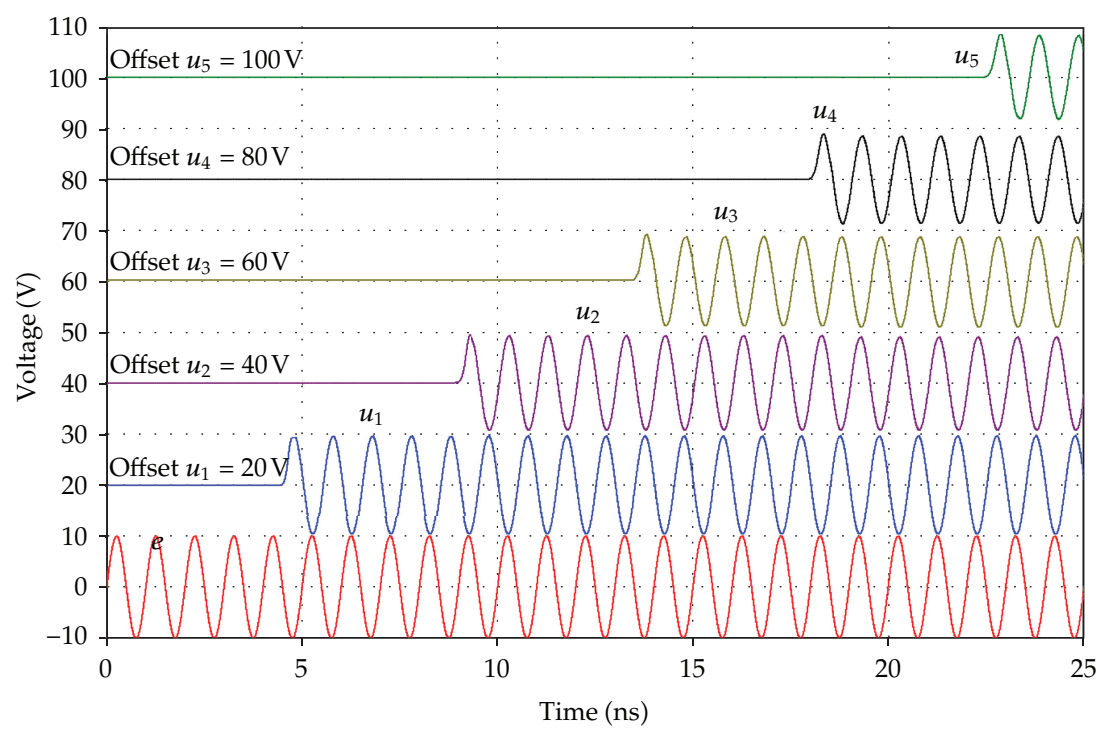

Figure 39: Transient analysis of RLCG ladder in Figure 38(c) and "measuring" of delay times $\tau_{1} \div \tau_{5}$ [see, also, (3)].

(ii) Delayed and slightly attenuated signals $u_{1} \div u_{5}$ with frequency $f_{0}$ and smooth transient intervals (Figure 39) are produced by uniform RLCG ladder terminated with characteristic impedance (The uniform ladder in Figure 38(c) consists of 1500 identical cells with impedances $Z_{1}\left(j \cdot 2 \pi \cdot f_{0}\right)=R / 20+j \cdot 2 \pi \cdot f_{0} \cdot L / 20$ and $Z_{2}\left(j \cdot 2 \pi \cdot f_{0}\right)=\left[Y_{2}\left(2 \pi \cdot f_{0}\right)\right]^{-1}$, where $Y_{2}\left(2 \pi \cdot f_{0}\right)=G / 10+j \cdot 2 \pi \cdot f_{0} \cdot C / 10$ (see Figure 37 and Table 1$)$. At frequency $f_{0}$ the characteristic impedance [8] of ladder is $Z_{c}(j \cdot 2 \pi \cdot f)=\left\{Z_{1}\left(j \cdot 2 \pi \cdot f_{0}\right) \cdot\left[Z_{1}\left(j \cdot 2 \pi \cdot f_{0}\right)+2 \cdot Z_{2}\left(j \cdot 2 \pi \cdot f_{0}\right)\right]\right\}^{1 / 2} \approx Z_{0}\left(j \cdot 2 \pi \cdot f_{0}\right)$ (it is close to the characteristic impedance of transmission line). Then, it can be shown that: (i) for $n=\overline{1,1500}$, the complex voltage at the end of the $n$th cell is $\approx$ $\exp \left\{-n \cdot \operatorname{acosh}\left[1+Z_{1}\left(j \cdot 2 \pi \cdot f_{0}\right) / Z_{2}\left(j \cdot 2 \pi \cdot f_{0}\right)\right]\right\} \cdot E\left(j \cdot 2 \pi \cdot f_{0}\right)\left[E\left(j \cdot 2 \pi \cdot f_{0}\right)\right.$ is the complex representative of $e(t)]$, and (ii) the time-delay at that place with respect to excitation is, $\operatorname{Im}\left\{n \cdot \operatorname{acosh}\left[1+Z_{1}\left(j \cdot 2 \pi \cdot f_{0}\right) / Z_{2}\left(j \cdot 2 \pi \cdot f_{0}\right)\right] /\left(2 \pi \cdot f_{0}\right)\right\} \approx 15.07 \cdot n$ [ps].) of the line at frequency $f_{0}$ (Figure $38(\mathrm{c})$ ) and not by real two-wire line. As will be seen, the transient response of real two-wire line, even with the same initial conditions and termination, is more complex.

(iii) A deeper insight into transient phenomena in real lines can be acquired, either by applying the numeric inverse Laplace transform of (A.17) with restricted number of poles/zeros or by numerical solving of linear, second-order, hyperbolic partial differential equations (2.19), telegraph equations, with specified initial and boundary conditions depending on line termination and excitation voltages in consecutive time intervals determined according to the line length. The method of lines seems to be the most appropriate for solving of hyperbolic and parabolic partial differential equations [11].

(iv) The PSPICE simulation method is applied herein only to facilitate the approximate steady-state analysis of two-wire lines with arbitrary loads and limited frequencyband signals, by using RLCG ladders as approximate network models of these 
lines, instead of resorting to numerical solving of partial differential equations or application of complex analytic methods.

To illustrate the complexity of transient phenomena in transmission lines let we consider two-wire line with length $\ell=4.5[\mathrm{~m}]$ and excitation $e(t)$ as in Figure 38(c) [see, also, (3)], terminated with its characteristic impedance at frequency $f_{0}$. At the moment of appearing of excitation at $t=0$, the line did not have any initial energy. Let us determine the solution $u(t, x)$ of the telegraph equation (2.19) in the interval $t \in[0, T]$, where $T=\ell /[2 \pi$. $\left.f_{0} / b\left(f_{0}\right)\right] \approx 22.5972[\mathrm{~ns}]$ is the perturbation propagation time from the line sending end to its receiving end, and $2 \pi \cdot f_{0} / b\left(f_{0}\right) \approx c_{0} /\left(\varepsilon_{r}\right)^{1 / 2} \approx 1.9913 \cdot 10^{8}[\mathrm{~m} / \mathrm{s}]$ is the propagation velocity. If we introduce substitution $t=\tau / A\left(f_{0}\right)\left\{A\left(f_{0}\right)=1 /\left[L^{\prime}\left(f_{0}\right) \cdot C^{\prime}\right]^{1 / 2}=1.9913 \cdot 10^{8}[\mathrm{~m} / \mathrm{s}]\right\}$ into the telegraph equation in $u(t, x)$ obtained from (2.19):

$$
\frac{\partial^{2} u(t, x)}{\partial x^{2}}=L^{\prime}\left(f_{0}\right) \cdot C^{\prime} \cdot \frac{\partial^{2} u(t, x)}{\partial t^{2}}+\left[L^{\prime}\left(f_{0}\right) \cdot G^{\prime}+C^{\prime} \cdot R^{\prime}\left(f_{0}\right)\right] \cdot \frac{\partial u(t, x)}{\partial t}+R^{\prime}\left(f_{0}\right) \cdot G^{\prime} \cdot u(t, x),
$$

we obtain the second-order, hyperbolic, partial differential equation (PDE) equivalent to (3.2):

$$
\begin{gathered}
\frac{\partial^{2} \bar{u}(\tau, x)}{\partial x^{2}}=\frac{\partial^{2} \bar{u}(\tau, x)}{\partial \tau^{2}}+C\left(f_{0}\right) \cdot \frac{\partial \bar{u}(\tau, x)}{\partial \tau}+B\left(f_{0}\right) \cdot \bar{u}(\tau, x) \\
u(t, x)=u\left[\frac{\tau}{A\left(f_{0}\right)}, x\right]=\bar{u}(\tau, x), \quad x \in[0, \ell] \\
A\left(f_{0}\right)=\frac{1}{\sqrt{L^{\prime}\left(f_{0}\right) \cdot C^{\prime}}}\left[\frac{\mathrm{m}}{\mathrm{s}}\right], \quad B\left(f_{0}\right)=R^{\prime}\left(f_{0}\right) \cdot G^{\prime}\left[\frac{1}{\mathrm{~m}^{2}}\right] \\
C\left(f_{0}\right)=R^{\prime}\left(f_{0}\right) \cdot \sqrt{\frac{C^{\prime}}{L^{\prime}\left(f_{0}\right)}}+G^{\prime} \cdot \sqrt{\frac{L^{\prime}\left(f_{0}\right)}{C^{\prime}}}\left[\frac{1}{\mathrm{~m}}\right]
\end{gathered}
$$

where $\tau \in\left[0, A\left(f_{0}\right) \cdot T\right][\mathrm{m}]$, that is, $\tau \in[0,4.5][\mathrm{m}]$. If $\bar{u}(\tau, x)=\left\{\exp \left[-(1 / 2) \cdot C\left(f_{0}\right) \cdot \tau\right]\right\} \cdot \overline{\bar{u}}(\tau, x)$, then from (3.3) the following PDE of Klein-Gordon's type [12] is obtained $\{\tau, x \in[0,4.5]$ [m]\}:

$$
\begin{gathered}
\frac{\partial^{2} \overline{\bar{u}}(\tau, x)}{\partial x^{2}}=\frac{\partial^{2} \overline{\bar{u}}(\tau, x)}{\partial \tau^{2}}+D\left(f_{0}\right) \cdot \overline{\bar{u}}(\tau, x) \\
D\left(f_{0}\right)=\frac{1}{4} \cdot\left(R^{\prime}\left(f_{0}\right) \cdot \sqrt{\frac{C^{\prime}}{L^{\prime}\left(f_{0}\right)}}-G^{\prime} \cdot \sqrt{\frac{L^{\prime}\left(f_{0}\right)}{C^{\prime}}}\right)^{2}=2.0241 \cdot 10^{-3}\left[\frac{1}{\mathrm{~m}^{2}}\right]
\end{gathered}
$$

which would be a pure wave equation if "diffusion" term $D\left(f_{0}\right) \cdot \overline{\bar{u}}(\tau, x)$ was not present, or in other words, if the line parameters satisfy the Heaviside's condition of distortionless at frequency $f_{0}$. 
Let we define, also, the following auxiliary function in $\tau$ and $x$ :

$$
\begin{gathered}
\overline{\bar{v}}(\tau, x)=\frac{\partial \overline{\bar{u}}(\tau, x)}{\partial \tau}=\left.\left[\frac{1}{2} \cdot C\left(f_{0}\right) \cdot u(t, x)+\frac{1}{A\left(f_{0}\right)} \cdot \frac{\partial u(t, x)}{\partial t}\right] \cdot \exp \left[E\left(f_{0}\right) \cdot t\right]\right|_{t=\tau / A\left(f_{0}\right)}, \\
E\left(f_{0}\right)=\frac{1}{2} \cdot\left[\frac{R^{\prime}\left(f_{0}\right)}{L^{\prime}\left(f_{0}\right)}+\frac{G^{\prime}}{C^{\prime}}\right]=8.959[\mathrm{MHz}] .
\end{gathered}
$$

If the excitation is $e(t)=E_{m} \cdot \sin \left(2 \pi \cdot f_{0} \cdot t+\varphi\right)$, then from (3.4) and (3.5) the system of two PDEs is produced to be solved in the interval $\tau, x \in[0,4.5][\mathrm{m}]$, by using of MATHCAD "Pdesolve" block:

$$
\begin{aligned}
& \frac{\partial \overline{\bar{u}}(\tau, x)}{\partial \tau}=\overline{\bar{v}}(\tau, x) \wedge \frac{\partial \overline{\bar{v}}(\tau, x)}{\partial \tau}=\frac{\partial^{2} \overline{\bar{u}}(\tau, x)}{\partial x^{2}}+D\left(f_{0}\right) \cdot \overline{\bar{u}}(\tau, x) \longleftarrow \text { The system of PDEs, } \\
& \overline{\bar{u}}(0, x)=\| \begin{array}{cl}
0 & \text { if } x>0 \\
e(0) & \text { otherwise }
\end{array} \wedge \overline{\bar{v}}(0, x) \\
& =\|\left[\frac{1}{2} \cdot C\left(f_{0}\right) \cdot e(0)+\frac{2 \pi \cdot f_{0} \cdot E_{m} \cdot \cos (\varphi)}{A\left(f_{0}\right)}\right] \quad \begin{array}{l}
\text { if } x>0 \\
\text { otherwise }
\end{array} \text { the initial conditions, } \\
& \overline{\bar{u}}(\tau, 0)=\exp \left[\frac{1}{2} \cdot C\left(f_{0}\right) \cdot \tau\right] \cdot e\left[\frac{\tau}{A\left(f_{0}\right)}\right] \wedge \overline{\bar{u}}(\tau, \ell)=0 \longleftarrow \text { the boundary conditions, } \\
& u(t, x)=\exp \left[-E\left(f_{0}\right) \cdot t\right] \\
& \cdot \overline{\bar{u}}\left[A\left(f_{0}\right) \cdot t, x\right] \longleftarrow \text { Solution of (3.2) in interval } t \in[0,22.59][\mathrm{ns}], x \in[0,4.5][\mathrm{m}] .
\end{aligned}
$$

In Figure 40 the solutions $u\left(t, x_{k}\right)$ of (3.2) in the interval $t \in[0, T]$ for $x_{k}=k \cdot \ell / 5[\mathrm{~m}]$ $(k=\overline{1,5})$ are depicted. Certainly the solutions of (3.2) for any $x \in[0, \ell]$ can be produced easily, also by using the relations (3.4)-(3.6).

In Figure 41 the pulse responses [i.e., the voltages $u_{k}(k=\overline{1,5})$ ] of the ladder in Figure 38(c) terminated with the characteristic impedance of two-wire line are depicted. The ladder is excited by pulsed emf $e(t)$ with amplitude 10 [V], frequency $f=10[\mathrm{MHz}]$, dutycycle 0.2 and rise and fall times equal 1 [ps]. The voltages $u_{k}(k=\overline{1,5})$ have overshoots, undershoots, and delay times close to those of the network in Figure 38(c) with continuous excitation of frequency $f_{0}=1[\mathrm{GHz}]$, in spite of the fact that frequency spectrum of the periodic, pulsed signal $e(t)$ has components $10 \cdot k[\mathrm{MHz}](k \in N)$ and that energetically significant part of spectrum is concentrated in the frequency range $f \in[0,150][\mathrm{MHz}]$.

\section{Conclusions}

In the paper new results are presented in incremental network modelling of Two-wire lines in the frequency range [0,3] [GHz], by uniform RLCG ladders with frequency-dependent $R L$ parameters, which are analyzed by using of the PSPICE. Some important frequency limitations of the proposed approach have been pinpointed, restricting the application 


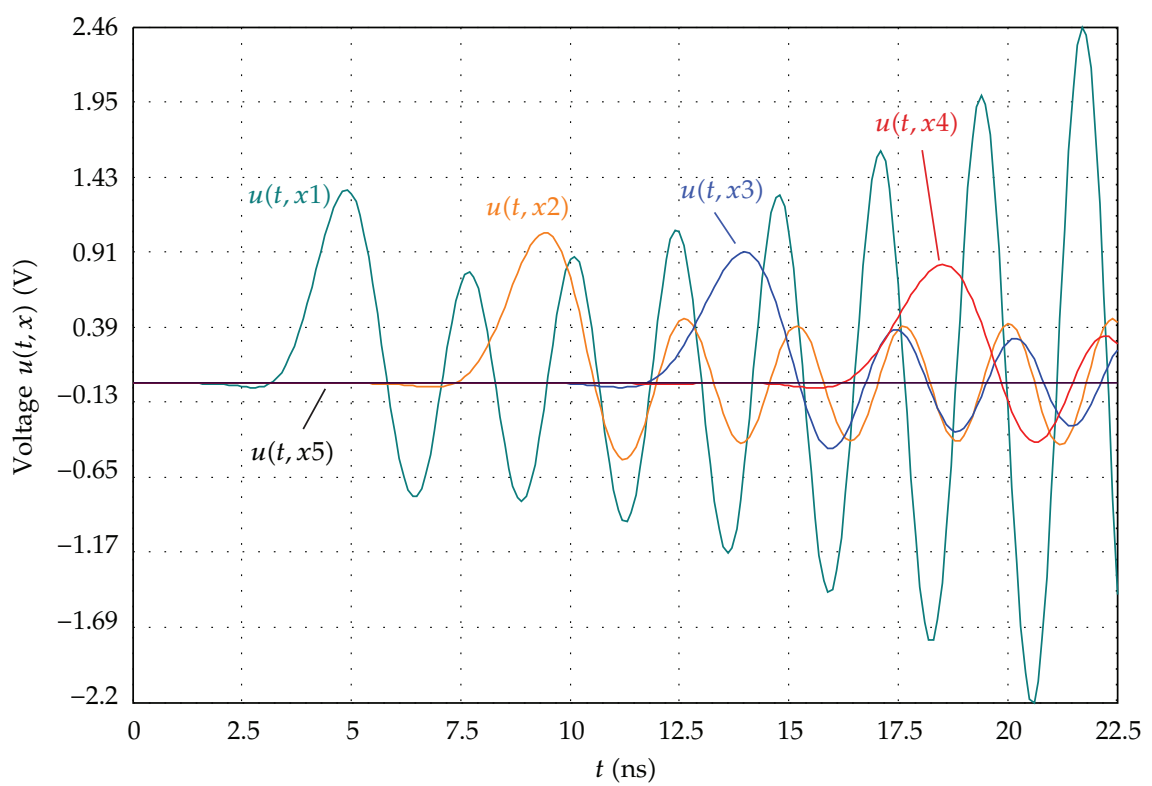

Figure 40: Transient voltages at five equidistant places on the two-wire line with length $\ell=4.5[\mathrm{~m}]\left(E_{m}=\right.$ $10[\mathrm{~V}]$ and $\varphi=0)$.

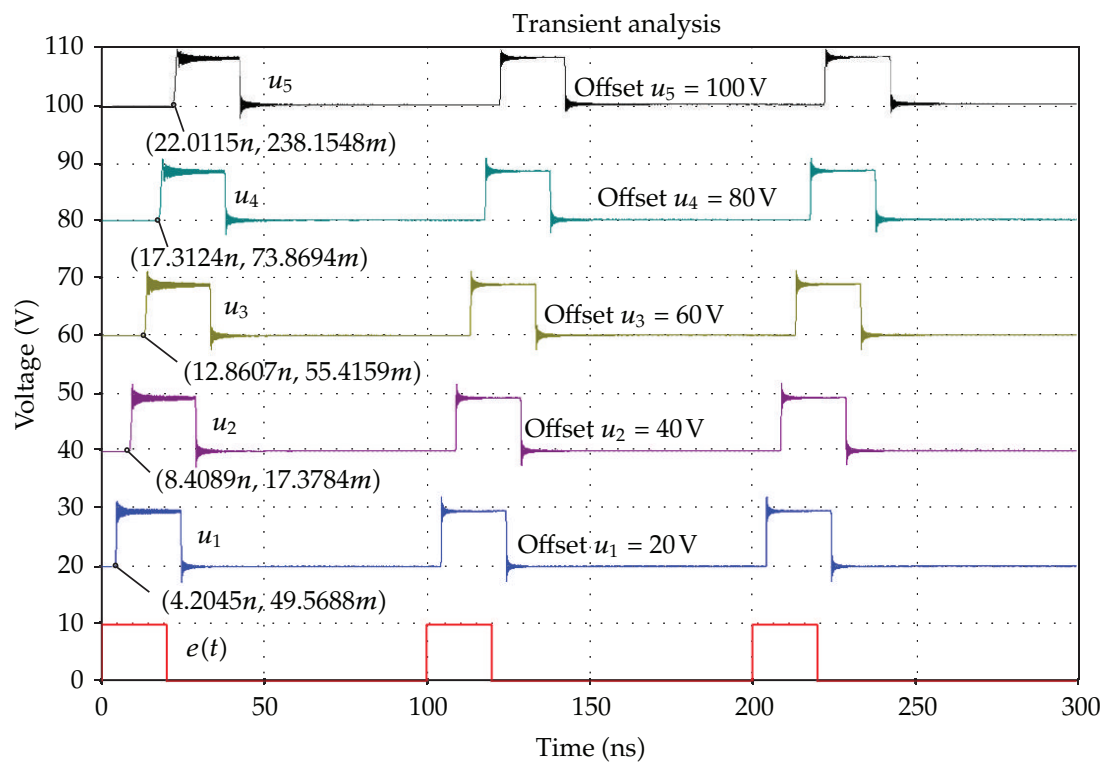

Figure 41: Pulsed responses in selected points on the uniform ladder in Figure 38(c).

of the developed models to the steady-state analysis of RLCG networks processing the limited-frequency-band signals. The basic intention of the approach considered herein is to circumvent solving of telegraph equations and application of the complex, numerically demanding procedures in determining two-wire line responses at selected set of equidistant points. The key to the modelling method applied is partition of the two-wire line in sufficiently short segments having defined maximum length, whereby couple of new 
polynomial approximations of line transcedental functions is introduced. It is proved that the strict equivalency between the short-line segments and their uniform ladder counterparts does not exist, but if some conditions are met, satisfactory approximations could be produced. This is illustrated by several examples of short and moderately long two-wire lines with different terminations, proving the good agreement between the exactly obtained steadystate results and those obtained by PSPICE simulation of RLCG ladders as the approximate incremental models of two-wire lines.

\section{Appendix}

By using of Kirchoff's voltage and current laws the following equlibrium equations can be written for the uniform transmission line depicted in Figure 2, no matter what its length $\ell$ or type is:

$$
\begin{gathered}
u\left(t, x+\frac{\delta x}{2}\right)-u(t, x)=-\frac{\delta x}{2} \cdot\left[L^{\prime} \cdot \frac{\partial i(t, x)}{\partial t}+R^{\prime} \cdot i(t, x)\right] \\
i(t, x+\delta x)-i(t, x)=-\delta x \cdot\left[C^{\prime} \cdot \frac{\partial u(t, x+\delta x / 2)}{\partial t}+G^{\prime} \cdot u\left(t, x+\frac{\delta x}{2}\right)\right], \\
u(t, x+\delta x)-u\left(t, x+\frac{\delta x}{2}\right)=-\frac{\delta x}{2} \cdot\left[L^{\prime} \cdot \frac{\partial i(t, x+\delta x)}{\partial t}+R^{\prime} \cdot i(t, x+\delta x)\right] .
\end{gathered}
$$

If $\delta x \rightarrow 0$, from (A.1)-(A.3) it immediately follows:

$$
\frac{\partial u(t, x)}{\partial x}=-L^{\prime} \cdot \frac{\partial i(t, x)}{\partial t}-R^{\prime} \cdot i(t, x), \quad \frac{\partial i(t, x)}{\partial x}=-C^{\prime} \cdot \frac{\partial u(t, x)}{\partial t}-G^{\prime} \cdot u(t, x)
$$

If $s$ is the complex frequency, let we suppose that the following conditions hold.

(a) Both $u(t, x)$ and $i(t, x)$ possess Laplace transform with respect to time,

$$
\begin{gathered}
U(s, x)=\ell[u(t, x)]=\int_{0^{-}}^{\infty} u(t, x) \cdot e^{-s \cdot t} \cdot \mathrm{d} t \\
I(s, x)=[i(t, x)]=\int_{0^{-}}^{\infty} i(t, x) \cdot e^{-s \cdot t} \cdot \mathrm{d} t .
\end{gathered}
$$

(b) Both $u(t, x)$ and $i(t, x)$ have continuous derivatives with respect to $x$.

(c) The following two integrals are uniformly convergent with respect to $x$ :

$$
\int_{0^{-}}^{\infty} \frac{\partial u(t, x)}{\partial x} \cdot e^{-s \cdot t} \cdot \mathrm{d} t, \quad \int_{0^{-}}^{\infty} \frac{\partial i(t, x)}{\partial x} \cdot e^{-s \cdot t} \cdot \mathrm{d} t
$$

(d) The initial conditions $u(0, x)$ and $i(0, x)$ are assumed for convenience to be zero for all $x$. 
Taking into account all conditions (a) $\div$ (d) and (A.1)-(A.6), it follows:

$$
\begin{gathered}
\ell\left[\frac{\partial u(t, x)}{\partial x}\right]=\int_{0^{-}}^{\infty} \frac{\partial u(t, x)}{\partial x} \cdot e^{-s \cdot t} \cdot \mathrm{d} t=\frac{\partial}{\partial x} \int_{0^{-}}^{\infty} u(t, x) \cdot e^{-s \cdot t} \cdot \mathrm{d} t=\frac{\partial U(s, x)}{\partial x} \\
\quad \ell\left[\frac{\partial i(t, x)}{\partial x}\right]=\int_{0^{-}}^{\infty} \frac{\partial i(t, x)}{\partial x} \cdot e^{-s \cdot t} \cdot \mathrm{d} t=\frac{\partial}{\partial x} \int_{0^{-}}^{\infty} i(t, x) \cdot e^{-s \cdot t} \cdot \mathrm{d} t=\frac{\partial I(s, x)}{\partial x}, \\
\frac{\partial U(s, x)}{\partial x}=-\left(R^{\prime}+L^{\prime} \cdot s\right) \cdot I(s, x), \quad \frac{\partial I(s, x)}{\partial x}=-\left(G^{\prime}+C^{\prime} \cdot s\right) \cdot U(s, x)
\end{gathered}
$$

wherefrom the equations describing voltage and current distribution in uniform line are readily produced, regardless to its length $\ell$ and/or terminal conditions (i.e., the generator and load impedances):

$$
\frac{\partial^{2} U(s, x)}{\partial x^{2}}+\Gamma^{2}(s) \cdot U(s, x)=0, \quad \frac{\partial^{2} I(s, x)}{\partial x^{2}}+\Gamma^{2}(s) \cdot I(s, x)=0,
$$

where $\Gamma(\sigma)=\sqrt{\left(R^{\prime}+L^{\prime} \cdot s\right) /\left(G^{\prime}+C^{\prime} \cdot s\right)}$ is propagation function of the line. The important parameter of any line is, also, its generalized characteristic impedance $Z_{0}(s)=$ $\sqrt{\left(R^{\prime}+L^{\prime} \cdot s\right) /\left(G^{\prime}+C^{\prime} \cdot s\right)}$. The line is distortionless if $R^{\prime} / L^{\prime}=G^{\prime} / C^{\prime}$ [7], and it is lossless when $R^{\prime}=0[\Omega / \mathrm{m}]$ and $G^{\prime}=0[\mathrm{~S} / \mathrm{m}]$. The general solution to the set of linear, homogeneous differential equations (A.10) reads

$$
U(s, x)=A_{1} \cdot \cosh (\Gamma \cdot x)+A_{2} \cdot \sinh (\Gamma \cdot x), \quad I(s, x)=B_{1} \cdot \cosh (\Gamma \cdot x)+B_{2} \cdot \sinh (\Gamma \cdot x),
$$

where the terms $A_{1}, A_{2}, B_{1}$, and $B_{2}$ are not the functions of $x$ and are determined from the boundary conditions. From (A.11) for $x=0$ we obtain, $A_{1}=U(s, 0)$ and $B_{1}=I(s, 0)$ and from (A.9) and (A.11); after differentiation in $x$; it follows, $A_{2}=-Z_{0}(s) \cdot I(s, 0)$ and $B_{2}=$ $-U(s, 0) / Z_{0}(s)$. Then (A.11) takes on the following form:

$$
\begin{gathered}
U(s, x)=U(s, 0) \cdot \cosh (\Gamma \cdot x)-Z_{0}(s) \cdot I(s, 0) \cdot \sinh (\Gamma \cdot x), \\
I(s, x)=I(s, 0) \cdot \cosh (\Gamma \cdot x)-\left[\frac{U(s, 0)}{Z_{0}(s)}\right] \cdot \sinh (\Gamma \cdot x) .
\end{gathered}
$$

Since for $x=\ell$ it holds:

$$
\begin{gathered}
U(s, \ell)=U(s, 0) \cdot \cosh (\Gamma \cdot \ell)-Z_{0}(s) \cdot I(s, 0) \cdot \sinh (\Gamma \cdot \ell), \\
I(s, \ell)=I(s, 0) \cdot \cosh (\Gamma \cdot \ell)-\left[\frac{U(s, 0)}{Z_{0}(s)}\right] \cdot \sinh (\Gamma \cdot \ell),
\end{gathered}
$$


then from (A.14) we obtain,

$$
\begin{gathered}
U(s, 0)=U(s, \ell) \cdot \cosh (\Gamma \cdot \ell)+Z_{0}(s) \cdot I(s, \ell) \cdot \sinh (\Gamma \cdot \ell), \\
I(s, 0)=I(s, \ell) \cdot \cosh (\Gamma \cdot \ell)+\left[\frac{U(s, \ell)}{Z_{0}(s)}\right] \cdot \sinh (\Gamma \cdot \ell) .
\end{gathered}
$$

If $Z_{L}(s)$ is the load impedance (termination) of the uniform, finite length line, then from (A.12)-(A.14) we finally produce voltage- and current-transmittances $M(s, x)$ and $N(s, x)$, respectively,

$$
\begin{gathered}
M(s, x)=\frac{U(s, x)}{U(s, 0)}=\frac{Z_{L}(s) \cdot \cosh [\Gamma \cdot(\ell-x)]+Z_{0}(s) \cdot \sinh [\Gamma \cdot(\ell-x)]}{Z_{L}(s) \cdot \cosh (\Gamma \cdot \ell)+Z_{0}(s) \cdot \sinh (\Gamma \cdot \ell)}, \\
N(s, x)=\frac{I(s, x)}{I(s, 0)}=\frac{Z_{L}(s) \cdot \sinh [\Gamma \cdot(\ell-x)]+Z_{0}(s) \cdot \cosh [\Gamma \cdot(\ell-x)]}{Z_{L}(s) \cdot \sinh (\Gamma \cdot \ell)+Z_{0}(s) \cdot \cosh (\Gamma \cdot \ell)} . \\
\text { Since } U(s, \ell)=Z_{L}(s) \cdot I(s, \ell), \text { then from }(\text { A.12)-(A.14) it follows, } \\
Z(s, 0)=\frac{U(s, 0)}{I(s, 0)}=\frac{Z_{L}(s) \cdot \cosh (\Gamma \cdot \ell)+Z_{0}(s) \cdot \sinh (\Gamma \cdot \ell)}{Z_{L}(s) \cdot \sinh (\Gamma \cdot \ell)+Z_{0}(s) \cdot \cosh (\Gamma \cdot \ell)} \cdot Z_{0}(s), \text { and generally, } \\
Z(s, x)=\frac{U(s, x)}{I(s, x)}=\frac{Z_{L}(s) \cdot \cosh [\Gamma \cdot(\ell-x)]+Z_{0}(s) \cdot \sinh [\Gamma \cdot(\ell-x)]}{Z_{L}(s) \cdot \sinh [\Gamma \cdot(\ell-x)]+Z_{0}(s) \cdot \cosh [\Gamma \cdot(\ell-x)]} \cdot Z_{0}(s), \quad x \in[0, \ell],
\end{gathered}
$$

where $Z(s, 0)$ is the input impedance of line and $Z(s, x)$ is the impedance at place $x$ seen towards the line end. From (A.17) and (A.18) we see that analysis of transmission line is equivalent to analysis of its segments terminated with impedances given with (A.18). The characteristic impedance and the propagation function of a distortionless line are $Z_{0}(s)=$ $\left(L^{\prime} / C^{\prime}\right)^{1 / 2}$ and $\Gamma(s)=\left(R^{\prime} \cdot G^{\prime}\right)^{1 / 2}+s \cdot\left(L^{\prime} \cdot C^{\prime}\right)^{1 / 2}$, respectively. And further if the line load impedance is $Z_{L}(s)=Z_{0}(s)$, then for all $x \in[0, \ell]$ it holds: $Z(s, x)=Z_{0}(s)=\left(L^{\prime} \cdot C^{\prime}\right)^{1 / 2}$ and $M(s, x)=N(s, x)=\exp [-\Gamma(s) \cdot x)]=\exp \left(-R^{\prime} \cdot G^{\prime} \cdot x\right) \cdot \exp \left[-x \cdot\left(L^{\prime} \cdot C^{\prime}\right)^{1 / 2} \cdot s\right]$. When the line parameters are constant, we will have $u(t, x)=\mathcal{L}^{-1}[U(s, x)]=\mathcal{L}^{-1}[M(s, x) \cdot U(s, 0)]=$ $\mathcal{L}^{-1}\left\{\exp \left(-R^{\prime} \cdot G^{\prime} \cdot x\right) \cdot \exp \left[-x \cdot\left(L^{\prime} \cdot C^{\prime}\right)^{1 / 2} \cdot s\right] \cdot U(s, 0)\right\}=\exp \left(-R^{\prime} \cdot G^{\prime} \cdot x\right) \cdot u\left[t-x \cdot\left(L^{\prime} \cdot C^{\prime}\right)^{1 / 2}, 0\right]$ and, also, $i(t, x)=\mathcal{L}^{-1}[I(s, x)]=\mathcal{L}^{-1}[N(s, x) \cdot I(s, 0)]=\mathcal{L}^{-1}\left\{\exp \left(-R^{\prime} \cdot G^{\prime} \cdot x\right) \cdot \exp \left[-x \cdot\left(L^{\prime} \cdot C^{\prime}\right)^{1 / 2}\right.\right.$. $s] \cdot I(s, 0)\}=\exp \left(-R^{\prime} \cdot G^{\prime} \cdot x\right) \cdot i\left[t-x \cdot\left(L^{\prime} \cdot C^{\prime}\right)^{1 / 2}\right]$, that is, voltage $u(t, x)$ and current $i(t, x)$ at place $x$ on distortionless line with constant parameters and load $Z_{L}(s)=\left(L^{\prime} / C^{\prime}\right)^{1 / 2}$ are as those on the line sending end, except for the time delay $\tau=x \cdot\left(L^{\prime} \cdot C^{\prime}\right)^{1 / 2}$ and the attenuation $\exp \left(R^{\prime} \cdot G^{\prime} \cdot x\right)$. By using of lossless, constant parameter line with resistive load $\left(L^{\prime} / C^{\prime}\right)^{1 / 2}$, it cannot be produced realistically even a relatively small signal delay. For example, pulse delay $\tau=5[\mathrm{~ms}]$ can be obtained from a lossless, constant parameter line with parameters $L^{\prime}=1.5[\mu \mathrm{H} / \mathrm{m}], C^{\prime}=18[\mathrm{pF} / \mathrm{m}]$, and the load resistance $\left(L^{\prime} / C^{\prime}\right)^{1 / 2}=500 / \sqrt{3}[\Omega]$ at distance $x=\tau \cdot\left(L^{\prime} \cdot C^{\prime}\right)^{-1 / 2} \approx 962.25[\mathrm{~km}]$ from the line sending end. But, if lossy, distortionless line is terminated with its characteristic impedance $Z_{0}(s)=\left(L^{\prime} / C^{\prime}\right)^{1 / 2}$, the attenuation factor $\exp [x$. $\left.\left(R^{\prime} \cdot G^{\prime}\right)^{1 / 2}\right]$ must, also, be taken into account. 


\section{Acknowledgment}

This work is supported in part by the Serbian Ministry of Science and Technological Development through Projects TR 32048 and III 41006.

\section{References}

[1] W. H. Hayt Jr., Engineering Electromagnetics, McGraw-Hill, New York, NY, USA, 1989.

[2] B. D. Popović, Electromagnetics, Građevinska Knjiga, Belgrade, Serbia, 1986.

[3] A. R. Đorđević, Electromagnetics, Akademska Misao, Belgrade, Serbia, 2008.

[4] S. Paull, Topics in Advanced Mathematics for Electronics Technology, John Wiley, New York, NY, USA, 1966.

[5] M. Abramowitz and I. Stegun, Handbook of Mathematical Functions, National Bureau of Standards, New York, NY, USA, 1970.

[6] R. S. Spencer and M. S. Ghausi, Introduction to Electronic Circuit Design, Prentice-Hall, New York, NY, USA, 2003.

[7] Y. H. Ku, Transient Circuit Analysis, D. Van Nostrand, New York, NY, USA, 1961.

[8] M. S. Ghausi and J. J. Kelly, Introduction to Distributed-Parameter Networks with Application to integrated Circuits, Holt, Reinhart and Winston, New York, NY, USA, 1968.

[9] A. P. Prudnikov, Y. A. Brychkov, and O. I. Marichev, Integrals and Series, vol. 1, Taylor \& Francis, New York, NY, USA, 2002.

[10] L. Weinberg, Network Analysis and Synthesis, McGraw-Hill, New York, NY, USA, 1961.

[11] D. S. Mitrinović and J. D. Kečkić, The Equations of Mathematical Physics, Građevinska Knjiga, Belgrade, Serbia, 1985.

[12] A. D. Polyanin, Handbook of Linear Partial Differential Equations for Engineers and Scientists, Chapman \& Hall/CRC, Boca Raton, Fla, USA, 2002. 


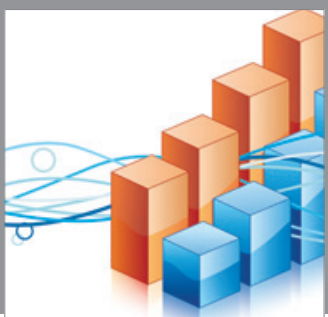

Advances in

Operations Research

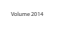

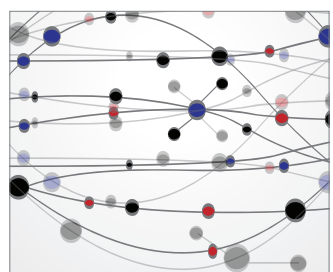

\section{The Scientific} World Journal
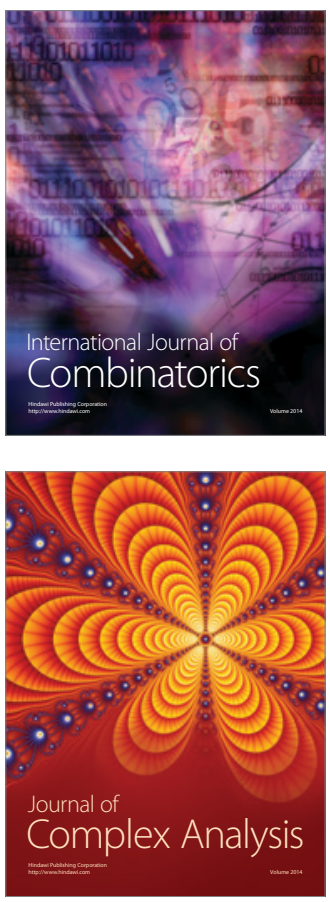

International Journal of

Mathematics and

Mathematical

Sciences
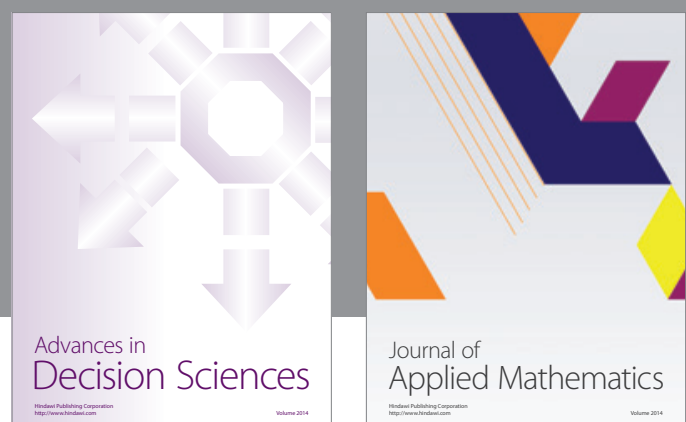

Journal of

Applied Mathematics
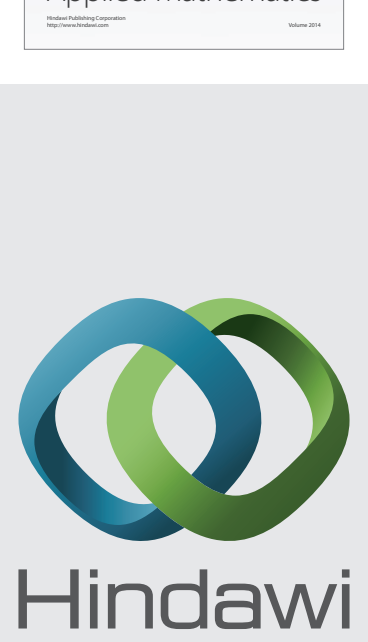

Submit your manuscripts at http://www.hindawi.com
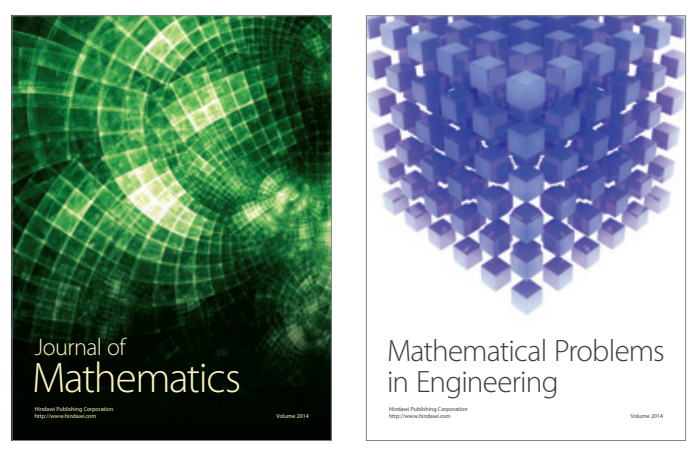

Mathematical Problems in Engineering
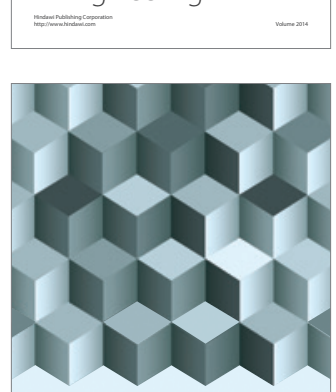

Journal of

Function Spaces
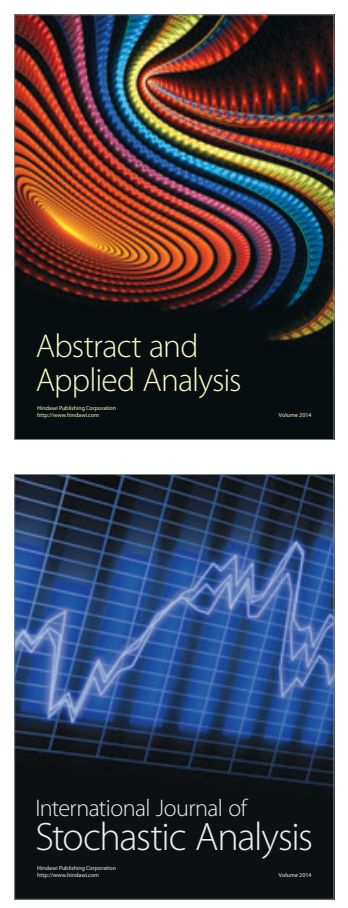

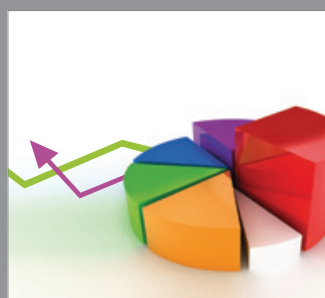

ournal of

Probability and Statistics

Promensencen
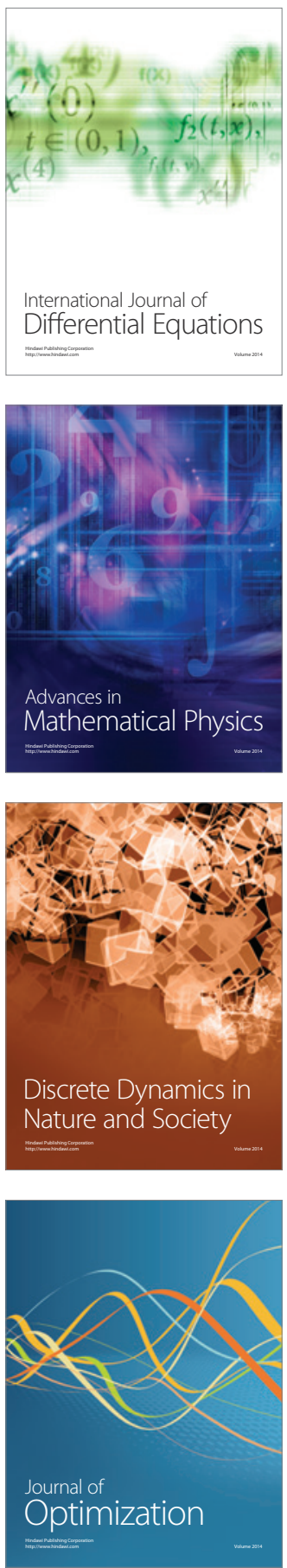